\title{
Electromagnetic form factors of the transition from the spin-3/2 $\Sigma$ to the $\Lambda$ hyperon
}

\author{
Olov Junker $\odot$, Stefan Leupold $\odot$, Elisabetta Perotti, and Timea Vitos \\ Institutionen för fysik och astronomi, Uppsala Universitet, Box 516, S-75120 Uppsala, Sweden
}

(Received 21 October 2019; published 16 January 2020)

\begin{abstract}
The three electromagnetic form factors for the transition from a $3 / 2^{+} \Sigma^{*}$ hyperon to the ground-state $\Lambda$ hyperon are studied. At low energies, combinations of the transition form factors can be deduced from Dalitz decays of the $\Sigma^{*}$ hyperon to $\Lambda$ plus an electron-positron pair. It is pointed out how more information can be obtained with the help of the self-analyzing weak decay of the $\Lambda$. In particular, it is shown that these transition form factors are complex quantities already in this kinematical region. Such measurements are feasible at hyperon factories such as, for instance, the Facility for Antiproton and Ion Research (FAIR). At higher energies, the transition form factors can be measured in electron-positron collisions. The transition form factors are related to decay distributions and differential cross sections. Using dispersion theory, the low-energy electromagnetic form factors for the $\Sigma^{*}$-to- $\Lambda$ transition are related to the pion vector form factor. The additionally required input, i.e., the two-pion- $\Sigma^{*}-\Lambda$ amplitudes, is determined from relativistic next-to-leading-order (NLO) baryon chiral perturbation theory, including the baryons from the octet and the decuplet. A poorly known NLO parameter is fixed to the experimental value of the $\Sigma^{*} \rightarrow \Lambda \gamma$ decay width. Pion rescattering is taken into account by using dispersion theory and solving a Muskhelishvili-Omnès equation. Subtracted and unsubtracted dispersion relations are discussed. However, in view of the fact that the transition form factors are complex quantities, the current data situation does not allow for a full determination of the subtraction constants. To reduce the number of free parameters, unsubtracted dispersion relations are used to make predictions for the transition form factors in the low-energy space- and timelike regions.
\end{abstract}

DOI: 10.1103/PhysRevC.101.015206

\section{INTRODUCTION AND SUMMARY}

Electromagnetic form factors have become an important tool to study the structure of strongly interacting objects; see, e.g., Refs. [1-12] and references therein. Depending on the invariant mass of the virtual photon, one achieves different resolutions and different degrees of freedom become relevant. At very large energies, one "sees" the minimal quark content of the probed object $[6,13,14]$. Asymptotic freedom causes a suppression of the influence of any nonminimal quark or gluon content of the probed state. At low energies, the dynamics of pions has an important influence on the shape of form factors. Dynamical chiral symmetry breaking causes the appearance of Goldstone bosons [15], the pions. Because they are much lighter than any other hadron, pions can be excited with energies so low that all other degrees of freedom are still frozen. Both aspects, dominance of minimal quark content at high energies and universal pion dynamics at low energies, are model-independent consequences of quantum chromodynamics (QCD). A complete description of a form factor must include both of these aspects.

Published by the American Physical Society under the terms of the Creative Commons Attribution 4.0 International license. Further distribution of this work must maintain attribution to the author(s) and the published article's title, journal citation, and DOI. Funded by $S C O A P^{3}$.
The purpose of Ref. [11] and of the present work is to provide the low-energy input for such a complete description of form factors in the hyperon sector. Here we extend the previous work of the Uppsala group $[11,16]$, in which dispersion theory is used to relate in a model-independent way isovector form factors of baryons to pion-baryon scattering amplitudes. In a second step, these scattering amplitudes are approximated by relativistic chiral perturbation theory $(\chi \mathrm{PT})$ including the baryon octet and decuplet as active degrees of freedom. Conceptually, this is close in spirit to Refs. [12,17-19]. On a more technical level, the rescattering of pions is treated differently in Refs. [12] and [11]. In Ref. [12], an N/D method ${ }^{1}$ is used; in Ref. [11] a Muskhelishvili-Omnès (MO) equation is solved. As has been demonstrated in Ref. [16] for the nucleon case, solving an MO equation with input from $\chi \mathrm{PT}$ up to next to leading order leads to better results when compared to a fully dispersive calculation [20,21]. For the case of hyperons, the use of dispersively reconstructed pion-baryon amplitudes is not an option because there are no direct data on pion-hyperon scattering. Therefore, we rely also in the present work on input from $\chi$ PT [7,22-25] and solve an MO equation. A combined use of dispersion theory and $\chi \mathrm{PT}$ has been pioneered in Ref. [26]; see also Ref. [27] for a brief review.

A general motivation for studying hyperon form factors has been provided in Ref. [11] in great detail. With the present

\footnotetext{
${ }^{1}$ We think that literally N/D just refers to "numerator over denominator," see Ref. [12] for details and further references.
} 
work, we extend the approach of Ref. [11] to electromagnetic form factors of hyperons with spin $3 / 2$. Our framework is suited for the determination of isovector form factors. Therefore, we focus in the present work on the only electromagnetic form factors of hyperons that are purely isovector (and involve a spin-3/2 state). These are the form factors for the transition of the lowest lying spin- $3 / 2$ decuplet state $\Sigma^{* 0}$ to the spin- $1 / 2$ ground state $\Lambda$.

In the timelike region, these transition form factors (TFFs) can be measured at low energies via the Dalitz decay $\Sigma^{* 0} \rightarrow$ $\Lambda e^{+} e^{-}$. It can be expected that these Dalitz decays will be addressed in the near future by the collaborations HADES [28] and PANDA [29] at the Facility for Antiproton and Ion Research (FAIR). Therefore, we regard our present work as very timely.

Concerning the $\Sigma^{*}-\Lambda$ transition, two distinct qualitative aspects are noteworthy; one is more case specific, one is universal. We start with the latter. Whenever pions are excited, they rescatter. In the isovector channel, the $p$-wave pion phase shift shows a relatively broad, essentially elastic resonance, the $\rho$ meson $[30,31]$. Thus, the universal pion dynamics gives rise to the coupling of the virtual photon to the $\rho$ meson. Phenomenologically, this is covered by the concept of vector meson dominance [32]. In the dispersive framework, this is covered by the pion phase shift and the pion vector form factor. We will explore the quantitative importance of these effects in the present paper.

There is a second aspect, however, which is also covered by our dispersive framework, but is typically missing in a vector meson dominance approach. This is the aspect that we called "more case specific." Being resonances, the $\Sigma^{*}$ hyperons are unstable. In particular, the $\Sigma^{* 0}$ can decay to $\Sigma^{ \pm} \pi^{\mp}$. This pair can rescatter into a $\Lambda$ and a real or virtual photon. Therefore, the TFFs are complex quantities in all kinematically allowed regimes: in the spacelike scattering region of $\Sigma^{* 0} e^{-} \leftrightarrow \Lambda e^{-}$; at the photon point $\Sigma^{* 0} \rightarrow \Lambda \gamma$; in the low-energy timelike Dalitz decay region of $\Sigma^{* 0} \rightarrow \Lambda e^{+} e^{-}$; and in the high-energy production region of $e^{+} e^{-} \rightarrow \Sigma^{* 0} \bar{\Lambda}$. This is in contrast to TFFs for hadrons that are stable with respect to the strong interaction. For stable hadrons, the TFFs are essentially real in all regimes except for the production region.

Complex form factors allow for nontrivial interference patterns between them. Those can be measured, e.g., with the help of the self-analyzing weak decays of the "stable" hyperons. In practice, this means that in the succession of the two decays $\Sigma^{* 0} \rightarrow \Lambda e^{+} e^{-}$and $\Lambda \rightarrow p \pi^{-}$, the angular distribution of the second decay contains interesting information about the interference of the TFFs. This information is accessible without involving the production process or the spin orientation of the $\Sigma^{* 0}$ and without determining the spin orientation of the proton $[33,34]$. On the other hand, in a strict vector meson dominance scenario, the $\Sigma^{* 0}$ couples just via a pointlike interaction to $\rho \Lambda$. There, a form factor can only become complex where the $\rho$ becomes unstable. This happens essentially only above the two-pion threshold. But in the Dalitz decay region of $\Sigma^{* 0} \rightarrow \Lambda e^{+} e^{-}$, the maximally possible dielectron invariant mass (" $\rho$-meson invariant mass") is $m_{\Sigma^{*}}-m_{\Lambda}<2 m_{\pi}$ [35]. Thus, in reality, the TFFs are complex but in a simple vector meson dominance approach they are real in the Dalitz decay region. We will also explore the quantitative importance of these effects. One peculiarity we observe is that even if the imaginary part of a TFF at the photon point is very small, it gets larger for the transition radius.

Ideally we would like to use subtracted dispersion relations, but the available experimental input is too scarce to allow for it. For the case at hand, there are three TFFs and therefore three complex-valued subtraction constants. For the time being, we choose to use unsubtracted dispersion relations; being aware of the large uncertainties they carry, we still expect to obtain results of the correct order of magnitude.

In the first part of the paper, we define the $\Sigma^{* 0}-\Lambda$ TFFs and relate them to several observables, accessible in different kinematical regions. Directly after we enter the core of the theoretical work, we derive the appropriate dispersion relations for pion-hyperon scattering amplitudes and TFFs. Finally, the results are presented. There are several Appendixes with various purposes. Appendix A clarifies how the individual contributions of meson and baryon dynamics influence the final results. The others complement the main text with technical details.

\section{TRANSITION FORM FACTORS AND OBSERVABLES}

Following essentially Ref. [5], we define three TFFs via

$$
\left\langle 0\left|j_{\mu}\right| \Sigma^{*} \bar{\Lambda}\right\rangle=e \bar{v}_{\Lambda}\left(p_{\Lambda}, \lambda\right) \Gamma_{\mu \nu}\left(p_{\Sigma^{*}}, p_{\Lambda}\right) u_{\Sigma^{*}}^{\nu}\left(p_{\Sigma^{*}}, \sigma\right)
$$

with

$$
\begin{aligned}
\Gamma^{\mu \nu}\left(p_{\Sigma^{*}}, p_{\Lambda}\right):= & -\left(\gamma^{\mu} q^{v}-\not q g^{\mu \nu}\right) m_{\Sigma^{*}} \gamma_{5} F_{1}\left(q^{2}\right) \\
& +\left(p_{\Sigma^{*}}^{\mu} q^{v}-p_{\Sigma^{*}} \cdot q g^{\mu \nu}\right) \gamma_{5} F_{2}\left(q^{2}\right) \\
& +\left(q^{\mu} q^{v}-q^{2} g^{\mu \nu}\right) \gamma_{5} F_{3}\left(q^{2}\right)
\end{aligned}
$$

and $q:=p_{\Sigma^{*}}+p_{\Lambda}$. Conventions for the spin-3/2 spinor $u^{\mu}$ are provided in Appendix B. The neutral spin-3/2 $\Sigma$ hyperon is denoted by $\Sigma^{*}$. The helicities (not spins!) of $\Sigma^{*}$ and $\bar{\Lambda}$ are called $\sigma$ and $\lambda$, respectively.

The TFFs defined via (1) are appropriate for a dispersive representation where we study formally the reaction $\Sigma^{*} \bar{\Lambda} \rightarrow$ $\pi^{+} \pi^{-} \rightarrow \gamma^{*}$. Physically, however, we study the reactions $e^{+} e^{-} \rightarrow \gamma^{*} \rightarrow \bar{\Sigma}^{*} \Lambda$ and $\Sigma^{*} \rightarrow \Lambda \gamma^{*} \rightarrow \Lambda e^{+} e^{-}$. In addition, if one wants to compare the results of the electromagnetic form factors for the transition $\Sigma^{*} \rightarrow \Lambda$ with the ones for $\Delta \rightarrow$ $N$, it is convenient to adapt to the conventions used in the $\Delta$ sector where mostly electroproduction is studied [6,7] and not Dalitz decays. Thus, one should also look at the reaction $e^{-} \Lambda \rightarrow e^{-} \Sigma^{*}$ or more formally $\Lambda \gamma^{*} \rightarrow \Sigma^{*}$. Therefore, we present the transition form factors also for other kinematical regimes.

In principle, the reactions $\Sigma^{*} \bar{\Lambda} \rightarrow \gamma^{*}, \gamma^{*} \rightarrow \bar{\Sigma}^{*} \Lambda$ and $\Sigma^{*} \rightarrow \Lambda \gamma^{*}$ are related by crossing symmetry. For $\Lambda \gamma^{*} \rightarrow$ $\Sigma^{*}$, one might involve charge conjugation and then again crossing symmetry.

For the amplitude relevant for the Dalitz decay, $\Sigma^{*} \rightarrow$ $\Lambda \gamma^{*}$, one finds

$$
\left\langle\Lambda\left|j_{\mu}\right| \Sigma^{*}\right\rangle=e \bar{u}_{\Lambda}\left(p_{\Lambda}, \lambda\right) \Gamma_{\mu \nu}\left(p_{\Sigma^{*}},-p_{\Lambda}\right) u_{\Sigma^{*}}^{\nu}\left(p_{\Sigma^{*}}, \sigma\right) .
$$

In practice, this leads to the very same expression as on the right-hand side of (2) but with $q:=p_{\Sigma^{*}}-p_{\Lambda}$. 
For the production amplitude $\gamma^{*} \rightarrow \bar{\Sigma}^{*} \Lambda$, one has to specify the meaning of the two-fermion bra state:

$$
\left\langle\bar{\Sigma}^{*} \Lambda|:=| \bar{\Sigma}^{*} \Lambda\right\rangle^{\dagger} .
$$

The structure corresponding to (2) would be $\Gamma^{\mu \nu}\left(-p_{\Sigma^{*}},-p_{\Lambda}\right)$, but it is not convenient to define $q$ as $-p_{\Sigma^{*}}-p_{\Lambda}$. Therefore, we rather provide a fully explicit version of the TFFs adapted to the production process:

$$
\begin{aligned}
\left\langle\bar{\Sigma}^{*} \Lambda\left|j_{\mu}\right| 0\right\rangle & =-e \bar{u}_{\Lambda}\left(p_{\Lambda}, \lambda\right) \tilde{\Gamma}_{\mu \nu}\left(p_{\Sigma^{*}}, p_{\Lambda}\right) v_{\Sigma^{*}}^{v}\left(p_{\Sigma^{*}}, \sigma\right), \\
\tilde{\Gamma}^{\mu \nu}\left(p_{\Sigma^{*}}, p_{\Lambda}\right) & :=\left(\gamma^{\mu} q^{\nu}-\not g g^{\mu \nu}\right) m_{\Sigma^{*}} \gamma_{5} F_{1}\left(q^{2}\right)+\left(p_{\Sigma^{*}}^{\mu} q^{\nu}-p_{\Sigma^{*}} \cdot q g^{\mu \nu}\right) \gamma_{5} F_{2}\left(q^{2}\right)+\left(q^{\mu} q^{\nu}-q^{2} g^{\mu \nu}\right) \gamma_{5} F_{3}\left(q^{2}\right)
\end{aligned}
$$

with $q:=p_{\Sigma^{*}}+p_{\Lambda}$.

Finally, we obtain for the excitation process

$$
\left\langle\Sigma^{*}\left|j_{\mu}\right| \Lambda\right\rangle=-e \bar{u}_{\Sigma^{*}}^{v}\left(p_{\Sigma^{*}}, \sigma\right) \tilde{\Gamma}_{\mu \nu}\left(p_{\Sigma^{*}},-p_{\Lambda}\right) u_{\Lambda}\left(p_{\Lambda}, \lambda\right)
$$

Here the pertinent expression for $\tilde{\Gamma}^{\mu v}$ agrees with the right-hand side of (6) provided one defines $q:=p_{\Sigma^{*}}-p_{\Lambda}$.

Next, we introduce linear combinations of $F_{1}, F_{2}$, and $F_{3}$, which correspond to TFFs with fixed helicity combinations. We denote them by $G_{m}(m=\sigma-\lambda=0, \pm 1)$ and find

$$
\begin{aligned}
G_{-1}\left(q^{2}\right) & :=\left[-m_{\Lambda}\left(m_{\Lambda}+m_{\Sigma^{*}}\right)+q^{2}\right] F_{1}\left(q^{2}\right)+\frac{1}{2}\left(m_{\Sigma^{*}}^{2}-m_{\Lambda}^{2}+q^{2}\right) F_{2}\left(q^{2}\right)+q^{2} F_{3}\left(q^{2}\right) \text { for } \sigma=-\frac{1}{2}, \lambda=+\frac{1}{2}, \\
G_{0}\left(q^{2}\right) & :=m_{\Sigma^{*}}^{2} F_{1}\left(q^{2}\right)+m_{\Sigma^{*}}^{2} F_{2}\left(q^{2}\right)+\frac{1}{2}\left(m_{\Sigma^{*}}^{2}-m_{\Lambda}^{2}+q^{2}\right) F_{3}\left(q^{2}\right) \text { for } \sigma=+\frac{1}{2}, \lambda=+\frac{1}{2},
\end{aligned}
$$

and

$$
G_{+1}\left(q^{2}\right):=m_{\Sigma^{*}}\left(m_{\Lambda}+m_{\Sigma^{*}}\right) F_{1}\left(q^{2}\right)+\frac{1}{2}\left(m_{\Sigma^{*}}^{2}-m_{\Lambda}^{2}+q^{2}\right) F_{2}\left(q^{2}\right)+q^{2} F_{3}\left(q^{2}\right) \quad \text { for } \quad \sigma=+\frac{3}{2}, \lambda=+\frac{1}{2} .
$$

In the following, we adopt the reference frame from Ref. [11] where the virtual photon is at rest, i.e., the $\Sigma^{*}-\bar{\Lambda}$ center-of-mass system, and where the $\Sigma^{*}$ is moving in the $z$ direction. In this frame, the three-momentum of the $\Sigma^{*}$ is given by $\vec{p}_{\Sigma^{*}}=p_{z} \vec{e}_{z}$ with

$$
p_{z}=\frac{\sqrt{\lambda\left(q^{2}, m_{\Sigma^{*}}^{2}, m_{\Lambda}^{2}\right)}}{2 \sqrt{q^{2}}}
$$

where we have introduced the Källén function

$$
\lambda(a, b, c):=a^{2}+b^{2}+c^{2}-2(a b+b c+a c) .
$$

We find

$$
\begin{aligned}
& \bar{v}_{\Lambda}\left(-p_{z}, 1 / 2\right) \Gamma_{3 v} u_{\Sigma^{*}}^{v}\left(p_{z},+1 / 2\right)=\bar{v}_{\Lambda} \gamma_{5} u_{\Sigma^{*}}^{3} \frac{2 q^{2}}{m_{\Sigma^{*}}^{2}-m_{\Lambda}^{2}+q^{2}} G_{0}\left(q^{2}\right), \\
& \bar{v}_{\Lambda}\left(-p_{z}, 1 / 2\right) \Gamma_{1 v} u_{\Sigma^{*}}^{v}\left(p_{z},-1 / 2\right)=\bar{v}_{\Lambda} \gamma_{5} u_{\Sigma^{*}}^{1} G_{-1}\left(q^{2}\right), \\
& \bar{v}_{\Lambda}\left(-p_{z}, 1 / 2\right) \Gamma_{1 v} u_{\Sigma^{*}}^{v}\left(p_{z},+3 / 2\right)=\bar{v}_{\Lambda} \gamma_{5} u_{\Sigma^{*}}^{1} G_{+1}\left(q^{2}\right) .
\end{aligned}
$$

The spinors on the right-hand side are evaluated with the same arguments as on the respective left-hand side. Note that in these relations the explicit "photon" indices 3 and 1 are covariant, not contravariant as is the case for the corresponding relations in Ref. [11]. This will lead to a sign change in Eq. (44) below as compared to the conventions of Ref. [11].

To make further contact with the existing literature, we relate our TFFs to the ones introduced in Ref. [6]. Therein, the transition from nucleon to $\Delta$ is considered. We replace $\Delta \rightarrow \Sigma^{*}$ and $N \rightarrow \Lambda$ to obtain our case at hand. The conventions for this process are provided in Eq. (7). It is convenient to define $Q^{2}:=-q^{2}$. Since one studies now reactions with $Q^{2}>0$, it is meaningful to introduce also $Q:=\sqrt{Q^{2}}$. The TFFs of Carlson [6] (in the following labeled with "Ca") are related to our TFFs by ${ }^{2}$

$$
G_{-}^{\mathrm{Ca}}=\frac{Q_{-}}{2 m_{\Lambda}} G_{+1}, \quad G_{+}^{\mathrm{Ca}}=\frac{Q_{-}}{2 \sqrt{3} m_{\Lambda}} G_{-1}, \quad G_{0}^{\mathrm{Ca}}=\frac{Q Q_{-}}{\sqrt{6} m_{\Lambda} m_{\Sigma^{*}}} G_{0}
$$

with $Q_{-}:=\sqrt{Q^{2}+\left(m_{\Lambda}-m_{\Sigma^{*}}\right)^{2}}$.

In Ref. [7], various conventions for the TFFs are related to each other, including the ones from Ref. [6]. With the help of (16) and Ref. [7], our TFFs can be easily related to any other TFF combinations and conventions.

\footnotetext{
${ }^{2}$ There is a mismatch between the conventions used in Ref. [6] and here. This is essentially based on the fact that we introduce our TFFs via the coupling of a virtual timelike photon to a spin-3/2 baryon and a spin-1/2 antibaryon where the latter has helicity $+1 / 2$; see (13)-(15). In Ref. [6], the TFFs are introduced via the coupling of a virtual spacelike photon to an incoming spin- $1 / 2$ baryon and an outgoing spin-3/2 baryon. The former has helicity $+1 / 2$. If one translates our case to the one in Ref. [6], our antibaryon turns to a baryon with helicity $-1 / 2$ and not $+1 / 2$. This sign change relates our TFF $G_{m}$ to Carlson's TFF $G_{-m}^{\mathrm{Ca}}$ for all $m=0, \pm 1$.
} 
At large spacelike momenta, i.e., for large $Q^{2}$, one finds the following asymptotic behavior from perturbative QCD [6]:

$$
\begin{aligned}
G_{-1}\left(-Q^{2}\right) & \sim \frac{1}{Q^{4}}, \quad G_{0,+1}\left(-Q^{2}\right) \sim \frac{1}{Q^{6}}, \\
F_{1}\left(-Q^{2}\right) & \sim \frac{1}{Q^{6}}, \quad F_{2,3}\left(-Q^{2}\right) \sim \frac{1}{Q^{8}} .
\end{aligned}
$$

Since we will provide only a low-energy representation for the various TFFs, one cannot expect to reproduce this asymptotic behavior without involving physics beyond the low-energy region. In general, this requires too much modeling. Nonetheless, it might be reasonable to aim for a representation where the TFFs fall off with $1 / Q^{4}$ at least. We will come back to this point below.

Pion-loop contributions to the TFFs can be most easily addressed for fixed helicity combinations. This favors the use of the TFFs (8)-(10). However, these combinations are subject to kinematical constraints; i.e., there is a kinematical point where these TFFs are not independent from each other. This happens at $q^{2}=\left(m_{\Lambda}+m_{\Sigma^{*}}\right)^{2}$, where $G_{+1}=G_{-1}=G_{0}\left(m_{\Lambda}+m_{\Sigma^{*}}\right) / m_{\Sigma^{*}}$. Dispersion relations should be formulated for constraint-free quantities [36,37]; otherwise, one might have to involve additional subtractions. The construction procedure of Refs. [36,37] leads to the TFFs of (2). Therefore, it can be useful to invert the relations (8)-(10), which yields

$$
\begin{aligned}
& F_{1}\left(q^{2}\right)=\frac{G_{+1}\left(q^{2}\right)-G_{-1}\left(q^{2}\right)}{\left(m_{\Sigma^{*}}+m_{\Lambda}\right)^{2}-q^{2}}, \\
& F_{2}\left(q^{2}\right)=\frac{2}{\lambda\left(m_{\Sigma^{*}}^{2}, m_{\Lambda}^{2}, q^{2}\right)}\left[-2 q^{2} G_{0}\left(q^{2}\right)+\left(m_{\Sigma^{*}} m_{\Lambda}-m_{\Lambda}^{2}+q^{2}\right) G_{+1}\left(q^{2}\right)+\left(m_{\Sigma^{*}}^{2}-m_{\Sigma^{*}} m_{\Lambda}\right) G_{-1}\left(q^{2}\right)\right], \\
& F_{3}\left(q^{2}\right)=\frac{2}{\lambda\left(m_{\Sigma^{*}}^{2}, m_{\Lambda}^{2}, q^{2}\right)}\left[\left(m_{\Sigma^{*}}^{2}-m_{\Lambda}^{2}+q^{2}\right) G_{0}\left(q^{2}\right)-m_{\Sigma^{*}}^{2}\left(G_{+1}\left(q^{2}\right)+G_{-1}\left(q^{2}\right)\right)\right]
\end{aligned}
$$

with the Källén function given in Eq. (12).

Let us turn now to observable production and decay processes. In terms of the TFFs, the decay width of $\Sigma^{*} \rightarrow \Lambda \gamma$ is given by

$$
\Gamma=\frac{e^{2}\left(m_{\Sigma^{*}}^{2}-m_{\Lambda}^{2}\right)}{96 \pi m_{\Sigma^{*}}^{3}}\left(m_{\Sigma^{*}}-m_{\Lambda}\right)^{2}\left(3\left|G_{+1}(0)\right|^{2}+\left|G_{-1}(0)\right|^{2}\right) .
$$

For the differential cross section of the reaction $e^{+} e^{-} \rightarrow \bar{\Sigma}^{*} \Lambda$ (see also [5]) we obtain in the center-of-mass (c.m.) frame and neglecting the electron mass

$$
\left(\frac{d \sigma}{d \Omega}\right)_{\text {c.m. }}\left(q^{2}, \theta\right)=\frac{e^{4}}{96 \pi^{2} q^{6}} p_{z} \frac{\sqrt{q^{2}}}{2}\left[q^{2}-\left(m_{\Sigma^{*}}-m_{\Lambda}\right)^{2}\right]\left\{\left(1+\cos ^{2} \theta\right)\left[3\left|G_{+1}\left(q^{2}\right)\right|^{2}+\left|G_{-1}\left(q^{2}\right)\right|^{2}\right]+\frac{4 q^{2}}{m_{\Sigma^{*}}^{2}} \sin ^{2} \theta\left|G_{0}\left(q^{2}\right)\right|^{2}\right\}
$$

with the center-of-mass momentum $p_{z}$ given in Eq. (11).

For the Dalitz decay distribution of $\Sigma^{*} \rightarrow \Lambda e^{+} e^{-}$, we provide one version, keeping the electron mass and one where only the kinematical velocity factor is kept. We introduce the electron velocity by

$$
\beta_{e}:=\sqrt{1-\frac{4 m_{e}^{2}}{q^{2}}}
$$

with the electron mass $m_{e}$. The doubly differential decay rate is given by

$$
\begin{aligned}
\frac{d \Gamma}{d q^{2} d \cos \theta}= & \frac{e^{4}}{(2 \pi)^{3} 96 m_{\Sigma^{*}}^{3} q^{2}} p_{z} \frac{\sqrt{q^{2}}}{2} \beta_{e}\left(\left(m_{\Sigma^{*}}-m_{\Lambda}\right)^{2}-q^{2}\right) \\
& \times\left\{\left(1+\cos ^{2} \theta+\frac{4 m_{e}^{2}}{q^{2}} \sin ^{2} \theta\right)\left[3\left|G_{+1}\left(q^{2}\right)\right|^{2}+\left|G_{-1}\left(q^{2}\right)\right|^{2}\right]+4\left(\sin ^{2} \theta+\frac{4 m_{e}^{2}}{q^{2}} \cos ^{2} \theta\right) \frac{q^{2}}{m_{\Sigma^{*}}^{2}}\left|G_{0}\left(q^{2}\right)\right|^{2}\right\} \\
\approx & \frac{e^{4}}{(2 \pi)^{3} 96 m_{\Sigma^{*}}^{3} q^{2}} p_{z} \frac{\sqrt{q^{2}}}{2} \beta_{e}\left(\left(m_{\Sigma^{*}}-m_{\Lambda}\right)^{2}-q^{2}\right)\left\{\left(1+\cos ^{2} \theta\right)\left[3\left|G_{+1}\left(q^{2}\right)\right|^{2}+\left|G_{-1}\left(q^{2}\right)\right|^{2}\right]+\frac{4 q^{2}}{m_{\Sigma^{*}}^{2}} \sin ^{2} \theta\left|G_{0}\left(q^{2}\right)\right|^{2}\right\} .
\end{aligned}
$$

Here $\theta$ denotes the angle between electron and $\Lambda$ in the rest frame of the electron-positron pair. If one calculates the integrated decay rate, the integration in $\theta$ ranges from $\pi$ to 0 such that the $\cos \theta$ integration ranges from -1 to +1 .

One should note that in the given decay the invariant mass $q^{2}$ of the photon is limited in the kinematical region

$$
4 m_{e}^{2} \leqslant q^{2} \leqslant\left(m_{\Sigma^{*}}-m_{\Lambda}\right)^{2}
$$

and so the factor $\left(m_{\Sigma^{*}}-m_{\Lambda}\right)^{2}-q^{2}$ will always be non-negative. If one blindly neglected the electron mass, one would obtain a divergent integrated decay rate. The phase-space factor $\beta_{e}$ and the proper integration range (23) ensure a physical, finite result. 
For later use, we also introduce a QED version of (22), which is supposed to describe the situation where the structure of hyperons is not resolved. In practice, we replace the TFF combinations by their $q^{2}=0$ expressions and make in this way also contact with the real photon case (19):

$$
\frac{d \Gamma_{\mathrm{QED}}}{d q^{2} d \cos \theta}:=\frac{e^{4}}{(2 \pi)^{3} 96 m_{\Sigma^{*}}^{3} q^{2}} p_{z} \frac{\sqrt{q^{2}}}{2} \beta_{e}\left(\left(m_{\Sigma^{*}}-m_{\Lambda}\right)^{2}-q^{2}\right)\left(1+\cos ^{2} \theta+\frac{4 m_{e}^{2}}{q^{2}} \sin ^{2} \theta\right)\left[3\left|G_{+1}(0)\right|^{2}+\left|G_{-1}(0)\right|^{2}\right] .
$$

Conceptually, small momenta go along with small $q^{2}$ and with treating the mass difference $m_{\Sigma^{*}}-m_{\Lambda}$ as small. By inspecting (22), we see that at small momenta the decay rate is dominated by the combination $3\left|G_{+1}\right|^{2}+\left|G_{-1}\right|^{2}$. In turn, (8) and (10) show that for low momenta the dominant contribution to $G_{+1}$ and $G_{-1}$ originates from $F_{1}$. At high momenta, $G_{-1}$ is dominant, as can be read off from (17); see also [6]. We deduce from (8) and (17) that it is again $F_{1}$ that dominates $G_{-1}$. Thus, in both limiting cases, low and high momenta, the TFF $F_{1}$ plays the dominant role.

More detailed access to the TFFs can be obtained by determining the angular distribution of the subsequent weak decay of the $\Lambda$; see also Ref. [34]. To this end, consider the decay $\Lambda \rightarrow p \pi^{-}$governed by the amplitude [35]

$$
\mathcal{M}_{\text {weak }}=G_{F} m_{\pi}^{2} \bar{u}_{p}\left(p_{1}\right)\left(A-B \gamma_{5}\right) u_{\Lambda}(p) .
$$

It is useful to introduce the asymmetry parameter

$$
\alpha_{\Lambda}:=\frac{2 \operatorname{Re}\left(T_{s}^{*} T_{p}\right)}{\left|T_{s}\right|^{2}+\left|T_{p}\right|^{2}}
$$

with the $s$-wave amplitude $T_{s}:=A$, the $p$-wave amplitude $T_{p}:=p_{\mathrm{f}} B /\left(E_{p}+m_{p}\right)$, and mass $m_{p}$, energy $E_{p}$, and momentum $p_{\mathrm{f}}$ of the proton in the rest frame of the decaying $\Lambda$ hyperon; i.e.,

$$
E_{p}=\frac{m_{\Lambda}^{2}+m_{p}^{2}-m_{\pi}^{2}}{2 m_{\Lambda}}
$$

and

$$
p_{\mathrm{f}}=\frac{\lambda^{1 / 2}\left(m_{\Lambda}^{2}, m_{p}^{2}, m_{\pi}^{2}\right)}{2 m_{\Lambda}}
$$

with the Källén function (12).

For the differential decay width of the whole four-body decay $\Sigma^{*} \rightarrow \Lambda e^{+} e^{-} \rightarrow p \pi^{-} e^{+} e^{-}$, one finds (neglecting again the electron mass where meaningful)

$$
\begin{aligned}
\frac{d \Gamma}{d q^{2} d \cos \theta d \Omega_{p}} \approx & \frac{e^{4}}{(2 \pi)^{4} 192 m_{\Sigma^{*}}^{3} q^{2}} p_{z} \frac{\sqrt{q^{2}}}{2} \beta_{e}\left(\left(m_{\Sigma^{*}}-m_{\Lambda}\right)^{2}-q^{2}\right) \operatorname{Br}_{\Lambda \rightarrow p \pi^{-}} \\
& \times\left\{\left(1+\cos ^{2} \theta\right)\left[3\left|G_{+1}\left(q^{2}\right)\right|^{2}+\left|G_{-1}\left(q^{2}\right)\right|^{2}\right]+\frac{4 q^{2}}{m_{\Sigma^{*}}^{2}} \sin ^{2} \theta\left|G_{0}\left(q^{2}\right)\right|^{2}\right. \\
& \left.+\frac{4 \sqrt{q^{2}}}{m_{\Sigma^{*}}} \alpha_{\Lambda} \operatorname{Im}\left[G_{0}\left(q^{2}\right) G_{-1}^{*}\left(q^{2}\right)\right] \cos \theta \sin \theta \sin \theta_{p} \sin \phi_{p}\right\}
\end{aligned}
$$

Here $\mathrm{Br}_{\Lambda \rightarrow p \pi^{-}}$denotes the branching ratio while $\theta_{p}$ and $\phi_{p}$ are the angles of the proton three-momentum measured in the rest frame of $\Lambda$. The coordinate system in this frame is defined by $\vec{q}$ pointing in the negative $z$ direction (i.e., in the rest frame of the virtual photon the $\Sigma^{*}$ and $\Lambda$ direction defines the positive $z$ axis) and the electron moves in the $x$ - $z$ plane with positive momentum projection on the $x$ axis. In this frame, $\theta_{p}$ is the angle of the proton momentum relative to the $z$ axis and $\phi_{p}$ is the angle between the $x$ axis and the projection of the proton momentum on the $x-y$ plane, i.e.,

$$
\begin{aligned}
\vec{p}_{p} & =p_{\mathrm{f}}\left(\sin \theta_{p} \cos \phi_{p}, \sin \theta_{p} \sin \phi_{p}, \cos \theta_{p}\right), \\
\vec{q} & =|\vec{q}|(0,0,-1), \\
\vec{p}_{e^{-}} \cdot \vec{e}_{y} & =0, \quad \vec{p}_{e^{-}} \cdot \vec{e}_{x}>0, \quad \vec{e}_{y}=\frac{\vec{p}_{e^{-}} \times \vec{q}}{\left|\vec{p}_{e^{-}}\right||\vec{q}|} .
\end{aligned}
$$

Note the subtlety that $\theta$ is measured in the rest frame of the virtual photon while $\Omega_{p}$ denotes angles in the rest frame of the $\Lambda$ hyperon. In terms of Lorentz invariant quantities, the angles are related to

$$
\begin{aligned}
p_{\Lambda} \cdot k_{e} & =-\frac{1}{2} \lambda^{1 / 2}\left(m_{\Sigma^{*}}^{2}, m_{\Lambda}^{2}, q^{2}\right) \cos \theta \\
\epsilon_{\mu \nu \alpha \beta} k_{e}^{\mu} p_{\Lambda}^{v} p_{p}^{\alpha} q^{\beta} & =-\frac{1}{2} \sqrt{q^{2}} \lambda^{1 / 2}\left(m_{\Sigma^{*}}^{2}, m_{\Lambda}^{2}, q^{2}\right) p_{\mathrm{f}} \sin \theta \sin \theta_{p} \sin \phi_{p}
\end{aligned}
$$


with $k_{e}:=p_{e^{-}}-p_{e^{+}}, q=p_{e^{-}}+p_{e^{+}}=p_{\Sigma^{*}}-p_{\Lambda}$ and the convention [38] for the Levi-Civita symbol:

$$
\epsilon_{0123}=-1 \text {. }
$$

A peculiar feature of (29) is the presence of the combination $\operatorname{Im}\left(G_{0} G_{-1}^{*}\right)$, which is nonzero even below the two-pion threshold. This is a consequence of the $\Sigma^{*}$ being unstable with respect to the strong interaction. This property plays a crucial role throughout the development of this paper and constitutes the main difference from the analogous $\Sigma-\Lambda$ case [11].

\section{DISPERSIVE MACHINERY}

Essentially this goes along the same lines as described in Refs. $[11,16]$. In particular, we use the same Omnès function,

$$
\Omega(s)=\exp \left\{s \int_{4 m_{\pi}^{2}}^{\infty} \frac{d s^{\prime}}{\pi} \frac{\delta\left(s^{\prime}\right)}{s^{\prime}\left(s^{\prime}-s-i \epsilon\right)}\right\},
$$

where $\delta$ denotes the pion $p$-wave phase shift [30,31]. The pion vector form factor $F_{\pi}^{V}$ is taken from Ref. [16] (see also Refs. [21,39,40]):

$$
F_{\pi}^{V}(s)=\left(1+\alpha_{V} s\right) \Omega(s) .
$$

For the pion phase shift from Ref. [31], a value of

$$
\alpha_{V}=0.12 \mathrm{GeV}^{-2}
$$

yields an excellent description of the data on the pion vector form factor from $\tau$ decays [41] for energies below $1 \mathrm{GeV}$; see Ref. [16].

\section{A. Dispersion relations}

Based on the asymptotic behavior (17), the three TFFs introduced in Eq. (2) satisfy unsubtracted dispersion relations

$$
F_{j}\left(q^{2}\right)=\int \frac{d s}{2 \pi i} \frac{\operatorname{disc} F_{j}(s)}{s-q^{2}}
$$

for $j=1,2,3$. Here "disc" denotes the discontinuity of the function $F_{j}$.

How does this translate to the TFFs $G_{m}$ introduced in Eqs. (8)-(10)? We can discuss this rather generally: If one defines two new TFFs, $A$ and $B$, via

$$
\begin{aligned}
& A\left(q^{2}\right):=F_{1}\left(q^{2}\right)+F_{2}\left(q^{2}\right), \\
& B\left(q^{2}\right):=F_{1}\left(q^{2}\right)+\frac{q^{2}}{s_{0}} F_{2}\left(q^{2}\right),
\end{aligned}
$$

one sees that they are subject to the kinematical constraint

$$
A\left(s_{0}\right)=B\left(s_{0}\right) .
$$

The dispersion relation for $A$ can be formulated without problems. For $B$, one obtains

$$
\begin{aligned}
B\left(q^{2}\right) & =\int \frac{d s}{2 \pi i} \frac{1}{s-q^{2}}\left[\operatorname{disc} F_{1}(s)+\frac{q^{2}}{s_{0}} \operatorname{disc} F_{2}(s)\right] \\
& =\int \frac{d s}{2 \pi i} \frac{1}{s-q^{2}}\left[\operatorname{disc} B(s)+\frac{q^{2}-s}{s_{0}} \operatorname{disc} F_{2}(s)\right] \\
& =\int \frac{d s}{2 \pi i} \frac{\operatorname{disc} B(s)}{s-q^{2}}-\frac{1}{s_{0}} \int \frac{d s}{2 \pi i} \operatorname{disc} F_{2}(s)
\end{aligned}
$$

This shows that in general one has to deal with an additional constant in a dispersive calculation of $B$. It is this constant that ensures that (38) holds. In addition, we have implicitly assumed that the dispersive integral over $\operatorname{disc} B$ actually converges.

For the TFFs $F_{j}$ that show the high-energy behavior (17), the situation is actually simpler. This high-energy behavior provides conditions for the integrals over disc $F_{j}$. In particular, the condition

$$
\lim _{Q^{2} \rightarrow \infty} Q^{2} F_{j}\left(-Q^{2}\right)=0
$$

leads to

$$
\int \frac{d s}{2 \pi i} \operatorname{disc} F_{j}(s)=0 .
$$

Thus, the additional constant in Eq. (39) vanishes. One obtains standard unsubtracted dispersion relations for $A$ and for $B$. In view of the relations (8)-(10), one can therefore conclude that also all the $G_{m}$ 's satisfy unsubtracted dispersion relations

$$
G_{m}\left(q^{2}\right)=\int \frac{d s}{2 \pi i} \frac{\operatorname{disc} G_{m}(s)}{s-q^{2}}
$$

for $m=0, \pm 1$.

\section{B. General considerations about the analytic structure}

At low energies, it can be expected that the $q^{2}$ behavior of the TFFs is determined by the lowest-energy states that can be excited. For the isovector TFFs that we study here, the lowest energetic states are pion pairs. Therefore, we can write in complete analogy to [11]

$$
\begin{aligned}
G_{m}\left(q^{2}\right)= & \frac{1}{12 \pi} \int_{4 m_{\pi}^{2}}^{\infty} \frac{d s}{\pi} \frac{T_{m}(s) p_{\mathrm{c} . \mathrm{m} .}^{3}(s) F_{\pi}^{V *}(s)}{s^{1 / 2}\left(s-q^{2}-i \epsilon\right)} \\
& +G_{m}^{\mathrm{anom}}\left(q^{2}\right)+\cdots,
\end{aligned}
$$

where the ellipsis denotes other intermediate states such as, for instance, four-pion or baryon-antibaryon states. The "anomalous" piece will be determined later. It is related to anomalous thresholds.

The pion-hyperon scattering amplitudes $T_{m}$ are obtained in a two-step procedure: In line with (13)-(15), we define first the reduced amplitudes

$$
\begin{aligned}
K_{ \pm 1}(s):= & -\frac{3}{4} \int_{0}^{\pi} d \theta \sin ^{2} \theta \\
& \times \frac{\mathcal{M}(s, \theta, 1 / 2 \pm 1,1 / 2)}{\bar{v}_{\Lambda}\left(-p_{z}, 1 / 2\right) \gamma_{5} u_{\Sigma^{*}}^{1}\left(p_{z}, 1 / 2 \pm 1\right) p_{\mathrm{c} . \mathrm{m} .}}, \\
K_{0}(s):= & -\frac{3}{2} \frac{m_{\Sigma^{*}}^{2}-m_{\Lambda}^{2}+s}{2 s} \int_{0}^{\pi} d \theta \sin \theta \cos \theta \\
& \times \frac{\mathcal{M}(s, \theta, 1 / 2,1 / 2)}{\bar{v}_{\Lambda}\left(-p_{z},+1 / 2\right) \gamma_{5} u_{\Sigma^{*}}^{3}\left(p_{z},+1 / 2\right) p_{\mathrm{c} . \mathrm{m}} .} .
\end{aligned}
$$

Here $p_{\mathrm{c} . \mathrm{m}}$. denotes the modulus of the momenta of the pions in the center-of-mass frame. We have introduced $\mathcal{M}(s, \theta, \sigma, \lambda)$ as the approximation to the Feynman amplitude for the reaction $\Sigma^{*} \bar{\Lambda} \rightarrow \pi^{+} \pi^{-}$. In practice, $\mathcal{M}(s, \theta, \sigma, \lambda)$ does not include the rescattering effect of the pions. This will be taken care of in the second step. In addition, we want to distinguish 
conceptually between processes with left-hand cut structures and purely polynomial terms. In practice, the reduced amplitudes $K$ originate from the left-hand cut structures only, while we denote the polynomial terms by $P$. All the formulas presented explicitly for $K$ apply also to $P$.

Pion rescattering is taken into account by solving a Muskhelishvili-Omnès equation $[42,43]$. The result is

$$
\begin{aligned}
T_{m}(s)= & K_{m}(s)+\Omega(s) P_{m}+T_{m}^{\mathrm{anom}}(s) \\
& +\Omega(s) s \int_{4 m_{\pi}^{2}}^{\infty} \frac{d s^{\prime}}{\pi} \frac{K_{m}\left(s^{\prime}\right) \sin \delta\left(s^{\prime}\right)}{\left|\Omega\left(s^{\prime}\right)\right|\left(s^{\prime}-s-i \epsilon\right) s^{\prime}} .
\end{aligned}
$$

As already spelled out, $K_{m}$ takes care of the left-hand cut structures. $P_{m}$ is a constant (per channel) that can be obtained ideally from a fit to data or estimated from $\chi$ PT. We have used here a once-subtracted dispersion relation. In principle, one could use more subtractions, which brings in a polynomial instead of a constant, but this would worsen the high-energy behavior. In the following, we will occasionally suppress the index $m$ when presenting generic formulas.

If there is an anomalous threshold, there might be an extra piece $T^{\text {anom }}(s)$ that is added to the amplitude. Such a situation can occur if the mass $m_{\text {exch }}$ of the exchanged state in the $t / u$ channel is "too light." For our reaction, the condition to have an anomalous threshold is [44]

$$
m_{\text {exch }}^{2}<\frac{1}{2}\left(m_{\Sigma^{*}}^{2}+m_{\Lambda}^{2}-2 m_{\pi}^{2}\right) .
$$

For the formal reaction $\Sigma^{*} \bar{\Lambda} \rightarrow 2 \pi$, one has to deal with the exchange of states carrying strangeness. In practice, we will take into account the exchange of $\Sigma$ and $\Sigma^{*}$ hyperons. The condition (46) does not hold for the $\Sigma^{*}$ exchange, ${ }^{3}$ but is satisfied for the $\Sigma$ exchange. In the latter case, the logarithm obtained from the partial-wave projection (44) requires a proper analytic continuation. If one takes the partial-wave projection literally (straight-line integral) as given in Eq. (44), then the obtained logarithm has a cut in the complex $s$ plane that intersects with the right-hand cut (unitarity cut), i.e., part of this cut lies on the physical Riemann sheet. To disentangle the cuts, one can define the cut of the logarithm such that it connects the branch point to the start of the unitarity cut by a straight line. The additional contribution $T^{\text {anom }}(s)$ takes care of the extra cut. A general discussion is provided in Appendix C.

To be more concrete, we note that the $p$-wave partial-wave projection of type (44) for a $t$ - or $u$-channel exchange process produces a term

$K(s)=g(s)-\frac{2 f(s)}{Y(s) \kappa^{2}(s)}+f(s) \frac{1}{\kappa^{3}(s)} \log \frac{Y(s)+\kappa(s)}{Y(s)-\kappa(s)}$

\footnotetext{
${ }^{3}$ It does not hold for any exchange of a many-particle state that contains a hyperon. The lightest such state would be a $\Lambda$ and one pion. Using that the $\Sigma^{*}$ is lighter than a $\Lambda$ and two pions, it is easy to check that the condition (46) is not satisfied for $m_{\mathrm{exch}} \geqslant m_{\Lambda}+m_{\pi}$.
}

with the functions $Y, \kappa$, and $\sigma$ defined in Appendix $\mathrm{C}$ for $m_{1} \rightarrow m_{\Sigma^{*}}, m_{2} \rightarrow m_{\Lambda}$. In addition, we have introduced $f(s)$, $g(s)$ as functions without cuts. These functions might have poles at kinematical thresholds, but they conspire such that no poles show up for $K$ as given in Eq. (47). If one expands the $\log$ function in powers of $\kappa / Y$, one sees that there are no poles for $\kappa \rightarrow 0$. Concrete formulas are given in Sec. V.

If one considers the standard logarithm with a cut along the real negative axis, then (47) is ill defined for $Y(s)=0$. This point lies on the unitarity cut if (46) is satisfied. To disentangle the two cuts, one starts with a proper analytic continuation of the logarithm along the unitarity cut. To this end, we introduce the following four points:

(1) At $s_{4}:=\left(m_{\Sigma^{*}}+m_{\Lambda}\right)^{2}$ we have $\kappa=0$. Above this point, i.e., for $s$ real and larger than $s_{4}$, there is the true scattering region. There, $\kappa$ is real and $Y$ is positive and larger than $\kappa$. The logarithm in Eq. (47) can be defined as the real-valued standard logarithm of positive numbers.

(2) At $s_{3}:=m_{\Sigma^{*}}^{2}+m_{\Lambda}^{2}+2 m_{\pi}^{2}-2 m_{\text {exch }}^{2}$ we have $Y=0$. For $s$ real and between $s_{3}$ and $s_{4}$, the function $\kappa$ is purely imaginary and $Y$ is still positive.

(3) At $s_{2}:=4 m_{\pi}^{2}$ we have $\kappa=0$. For $s$ real and between $s_{2}$ and $s_{3}$, the function $\kappa$ is purely imaginary and $Y$ is negative.

(4) At $s_{1}:=\left(m_{\Sigma^{*}}-m_{\Lambda}\right)^{2}$ we have $\kappa=0$. For $s$ real and between $s_{1}$ and $s_{2}$, the function $\kappa$ is real (and $Y$ is negative).

For the case of a $\Sigma$ exchange, we have $0<s_{1}<s_{2}<s_{3}<$ $s_{4}$. The function $K$ in Eq. (47) that enters finally (45) is then defined on the relevant part of the real axis by

$$
K(s):=g(s)-\frac{2 f(s)}{Y(s) \kappa^{2}(s)}+\frac{f(s)}{\kappa^{3}(s)} \log \frac{Y(s)+\kappa(s)}{Y(s)-\kappa(s)}
$$

for $s>s_{4}$,

$$
K(s):=g(s)-\frac{2 f(s)}{Y(s) \kappa^{2}(s)}+\frac{2 f(s)}{\kappa^{2}(s)|\kappa(s)|} \arctan \frac{|\kappa(s)|}{Y(s)}
$$

for $s_{3}<s<s_{4}$, and

$$
\begin{aligned}
K(s):= & g(s)-\frac{2 f(s)}{Y(s) \kappa^{2}(s)} \\
& +\frac{2 f(s)}{\kappa^{2}(s)|\kappa(s)|}\left(\arctan \frac{|\kappa(s)|}{Y(s)}+\pi\right)
\end{aligned}
$$

for $s_{2}<s<s_{3}$. Here the standard logarithm for positive real numbers is used and the arctan function with values between $-\pi / 2$ and $\pi / 2$. Note that at the two-pion threshold $s=s_{2}$ the quantity $K(s)$ of (50) diverges $\sim 2 \pi f(s) /\left(\kappa^{2}(s)|\kappa(s)|\right) \sim$ $1 / \sigma^{3}(s)$, but the product $K(s) \sin \delta(s)$ in Eq. (45) remains finite due to $\sin \delta(s) \sim \sigma^{3}(s)$ for the $p$-wave pion phase shift [30,31]. $K(s)$ also appears in the combination $K(s) p_{\text {c.m. }}^{3}(s)$ in Eq. (43), which remains also finite. 
The second issue is the definition of $T^{\text {anom; }}$ see also the discussion in Appendix C. The branch points of the logarithm in Eq. (47) are defined by $Y^{2}(s)=\kappa^{2}(s)$. They are located at

$$
s_{ \pm}=-\frac{1}{2} m_{\mathrm{exch}}^{2}+\frac{1}{2}\left(m_{\Sigma^{*}}^{2}+m_{\Lambda}^{2}+2 m_{\pi}^{2}\right)-\frac{m_{\Sigma^{*}}^{2} m_{\Lambda}^{2}-m_{\pi}^{2}\left(m_{\Sigma^{*}}^{2}+m_{\Lambda}^{2}\right)+m_{\pi}^{4}}{2 m_{\mathrm{exch}}^{2}} \mp \frac{\lambda^{1 / 2}\left(m_{\Sigma^{*}}^{2}, m_{\mathrm{exch}}^{2}, m_{\pi}^{2}\right) \lambda^{1 / 2}\left(m_{\mathrm{exch}}^{2}, m_{\Lambda}^{2}, m_{\pi}^{2}\right)}{2 m_{\mathrm{exch}}^{2}} .
$$

We take $s_{+}$as the solution that has a positive imaginary part for small values of $m_{\Sigma^{*}}^{2}$. If one replaces $m_{\Sigma^{*}}^{2}$ by $m_{\Sigma^{*}}^{2}+i \epsilon$ and follows the motion of $s_{+}$for increasing values of $m_{\Sigma^{*}}^{2}$, then $s_{+}$moves towards the real axis and intersects with the unitarity cut where (46) turns to an equality. For larger values of $m_{\Sigma^{*}}^{2}$ one finds $s_{+}$in the lower half plane of the first Riemann sheet. This is the situation for the physical value of $m_{\Sigma^{*}}^{2}$ for the case $m_{\mathrm{exch}}^{2}=m_{\Sigma}^{2}$. Thus, we have

$s_{+}=-\frac{1}{2} m_{\Sigma}^{2}+\frac{1}{2}\left(m_{\Sigma^{*}}^{2}+m_{\Lambda}^{2}+2 m_{\pi}^{2}\right)-\frac{m_{\Sigma^{*}}^{2} m_{\Lambda}^{2}-m_{\pi}^{2}\left(m_{\Sigma^{*}}^{2}+m_{\Lambda}^{2}\right)+m_{\pi}^{4}}{2 m_{\Sigma}^{2}}-i \frac{\lambda^{1 / 2}\left(m_{\Sigma^{*}}^{2}, m_{\Sigma}^{2}, m_{\pi}^{2}\right)\left[-\lambda\left(m_{\Sigma}^{2}, m_{\Lambda}^{2}, m_{\pi}^{2}\right)\right]^{1 / 2}}{2 m_{\Sigma}^{2}}$

with positive square roots.

The anomalous contribution that enters (45) is then given by

$$
T^{\text {anom }}(s)=\Omega(s) s \int_{0}^{1} d x \frac{d s^{\prime}(x)}{\mathrm{d} x} \frac{1}{s^{\prime}(x)-s} \frac{2 f\left(s^{\prime}(x)\right)}{\left[-\lambda\left(s^{\prime}(x), m_{\Sigma^{*}}^{2}, m_{\Lambda}^{2}\right)\right]^{1 / 2} \kappa^{2}\left(s^{\prime}(x)\right)} \frac{t\left(s^{\prime}(x)\right)}{\Omega\left(s^{\prime}(x)\right) s^{\prime}(x)}
$$

with the straight-line path

$$
s^{\prime}(x):=(1-x) s_{+}+x 4 m_{\pi}^{2}
$$

that connects the branch point (52) of the logarithm of (47) and the branch point $4 m_{\pi}^{2}$ of the unitarity cut.

One also needs the scattering amplitude $t(s)$ in the complex plane. Following Ref. [45], one could use an analytic continuation of the amplitude as constructed from $\chi \mathrm{PT}$ and unitarized by the inverse amplitude method. This representation does not show a decent high-energy behavior. Therefore, we will use it only for the anomalous part. There the whole integration region is rather close to the two-pion threshold. Therefore, an expression from $\chi \mathrm{PT}$ or a unitarized version thereof should be sufficiently close to the true scattering amplitude. We take from Ref. [45] the following expressions (extended to the complex plane). The approximation from $\chi \mathrm{PT}$ is given by

$$
t_{\chi \mathrm{PT}}(s) \approx t_{2}(s)+t_{4}(s)
$$

and its unitarized version is

$$
t_{\mathrm{IAM}}(s)=\frac{t_{2}^{2}(s)}{t_{2}(s)-t_{4}(s)}
$$

with

$$
\begin{aligned}
t_{2}(s) & =\frac{s \sigma^{2}}{96 \pi F_{0}^{2}} \\
t_{4}(s) & =\frac{t_{2}(s)}{48 \pi^{2} F_{0}^{2}}\left\{s\left(\bar{l}+\frac{1}{3}\right)-\frac{15}{2} m_{\pi}^{2}-\frac{m_{\pi}^{4}}{2 s}\left[41-2 L_{\sigma}\left(73-25 \sigma^{2}\right)+3 L_{\sigma}^{2}\left(5-32 \sigma^{2}+3 \sigma^{4}\right)\right]\right\}-\hat{\sigma}(s) t_{2}^{2}(s) \\
L_{\sigma} & =\frac{1}{\sigma^{2}}\left(\frac{1}{2 \sigma} \log \frac{1+\sigma}{1-\sigma}-1\right)
\end{aligned}
$$

The functions $\sigma(s)$ and $\hat{\sigma}(s)$ are defined in Eqs. (C6) and (C9), respectively. Note that there is no square root ambiguity in the definition of $\sigma$ since all expressions are even in $\sigma \rightarrow-\sigma$. The square root appearing in the definition of the function $\hat{\sigma}$ has its cut on the negative real axis. Then the function $\hat{\sigma}$ has the unitarity cut (and also a cut along the negative real axis).

The value for the pion decay constant in the chiral limit $F_{0}$ is taken from the ratio $F_{\pi} / F_{0}=1.064(7)$, where $F_{\pi}=$ 92.28(9) $\mathrm{MeV}$ is the pion decay constant at the physical point. In the original paper [45], the low-energy constant $\bar{l}=5.73(8)$ has been adjusted such as to reproduce the position of the pole of the $\rho$-meson resonance on the second Riemann sheet. In this work, instead we use $\bar{l}=6.47$, which is obtained by requiring agreement between the pion $p$-wave phase shifts from (56) and from Ref. [31] around the two-pion threshold.

Finally, we provide the anomalous piece of the TFFs. As described in Appendix $\mathrm{C}$, one can relate the anomalous piece of the TFF to the anomalous piece of $T-K$. Therefore, we obtain

$$
\begin{aligned}
G_{m}^{\mathrm{anom}}\left(q^{2}\right)= & \frac{1}{12 \pi} \int_{0}^{1} d x \frac{d s^{\prime}(x)}{d x} \frac{1}{s^{\prime}(x)-q^{2}} \\
& \times \frac{f_{m}\left(s^{\prime}(x)\right) s^{\prime}(x) F_{\pi}^{V}\left(s^{\prime}(x)\right)}{-4\left[-\lambda\left(s^{\prime}(x), m_{\Sigma^{*}}^{2}, m_{\Lambda}^{2}\right)\right]^{3 / 2}} .
\end{aligned}
$$


Note that the Omnès function (33) that enters the pion vector form factor (34) is defined everywhere on the first Riemann sheet via the pion phase shift along the right-hand cut. Therefore, (60) can be calculated without problems.

Note that without any anomalous piece the TFF integral in (43) would be real below the two-pion threshold. However, the TFF should be complex because the $\Sigma^{*}$ is unstable. The imaginary part emerges from the following process: Irrespective of the invariant mass of the photon, the $\Sigma^{*}$ can decay to a pion and a $\Sigma$. These states can rescatter into a $\Lambda$ and a real or virtual photon. The anomalous pieces take care of this physical process.

A remark on why we include only the pions as intermediate states and not the kaons is in order. When writing down a dispersion relation, thresholds and anomalous cuts have to be identified and ranked according to their relevance. The dispersive representation ensures that the influence of highenergy inelasticities is suppressed, provided that we limit ourselves to low values of $q^{2}$. The two-pion threshold, which starts at $\left(2 m_{\pi}\right)^{2} \approx 0.08 \mathrm{GeV}^{2}$, is the most important. The branch point of the anomalous cut for the $\Sigma$-pion-pion triangle lies in the vicinity, i.e., at $s_{+} \approx(0.07-0.03 i) \mathrm{GeV}^{2}$. To increase the validity of our approach, we include the full pion rescattering with the $\rho$-meson appearing in the phase shift, but this does not mean that one should trust the calculations up to the $\rho$-meson mass. But since the $\rho$ meson has such a large impact, one cannot neglect its influence, not even at low energies. This is very different from the triangle including kaons. As already pointed out in Ref. [44], hyperon scattering with nucleon exchange leads to an anomalous threshold. We have checked that for the reaction $\Sigma^{*} \bar{\Lambda} \rightarrow K \bar{K}$ with nucleon exchange, the anomalous threshold lies at $\approx 0.7 \mathrm{GeV}^{2}$, i.e., significantly lower than the two-kaon threshold, but rather far away from the thresholds of the pion triangles that we have considered. There is one possibility that would spoil our argument, namely if the strength of the kaon triangle would be much larger than the pion triangle. To compare these two, one can look at the strengths of the vertices for the scattering processes $\Sigma^{*} \bar{\Lambda} \rightarrow \pi^{+} \pi^{-}$with $\Sigma^{-}$exchange versus $\Sigma^{*} \bar{\Lambda} \rightarrow$ $K^{+} K^{-}$with proton exchange. The vertices containing the $\Sigma^{*}$ have the very same strength. The vertex for $\Sigma^{-} \bar{\Lambda} \pi^{+}$yields a strength of $D=0.8$ while the corresponding one for $p \bar{\Lambda} K^{-}$ contributes with $(D+3 F) / 2=1.09$. Thus, the kaon triangle has similar strength as the pion triangle but much higher lying thresholds. Therefore, we leave out the diagrams with the kaons.

\section{Subtracted dispersion relations}

Though the intermediate states not explicitly considered in Eq. (43) might have a minor influence on the shape of the TFFs at low energies, it is likely that they have an impact on the overall size; see, e.g., the discussion in Refs. [11,16,21]. A way to enhance the importance of the low-energy region in a dispersive integral is the use of a subtracted dispersion relation. The most conservative approach that does not make use of any high-energy input is to start again from the unconstrained TFFs $F_{i}$. A subtracted dispersion relation reads

$$
\begin{aligned}
F_{i}\left(q^{2}\right)= & F_{i}(0)+\frac{q^{2}}{12 \pi} \int_{4 m_{\pi}^{2}}^{\Lambda^{2}} \frac{d s}{\pi} \frac{T_{i}(s) p_{\mathrm{c} . \mathrm{m} .}^{3}(s) F_{\pi}^{V *}(s)}{s^{3 / 2}\left(s-q^{2}-i \epsilon\right)} \\
& +F_{i}^{\mathrm{anom}}\left(q^{2}\right)
\end{aligned}
$$

for $i=1,2,3$. The last, "anomalous" piece will be specified below.

In principle, the scattering amplitudes $T_{i}$ are again given by (45) but now we need the amplitudes $K_{i}, i=1,2,3$ as input. They are obtained from $K_{+1,0,-1}$ in the same way as the TFFs $F_{i}$ are obtained from $G_{+1,0,-1}$, i.e.,

$$
\begin{aligned}
K_{1}(s)= & \frac{K_{+1}(s)-K_{-1}(s)}{\left(m_{\Sigma^{*}}+m_{\Lambda}\right)^{2}-s} \\
K_{2}(s)= & \frac{2}{\lambda\left(m_{\Sigma^{*}}^{2}, m_{\Lambda}^{2}, s\right)}\left[-2 s K_{0}(s)\right. \\
& +\left(m_{\Sigma^{*}} m_{\Lambda}-m_{\Lambda}^{2}+s\right) K_{+1}(s) \\
& \left.+\left(m_{\Sigma^{*}}^{2}-m_{\Sigma^{*}} m_{\Lambda}\right) K_{-1}(s)\right], \\
K_{3}(s)= & \frac{2}{\lambda\left(m_{\Sigma^{*}}^{2}, m_{\Lambda}^{2}, s\right)}\left[\left(m_{\Sigma^{*}}^{2}-m_{\Lambda}^{2}+s\right) K_{0}(s)\right. \\
& \left.-m_{\Sigma^{*}}^{2}\left(K_{+1}(s)+K_{-1}(s)\right)\right] .
\end{aligned}
$$

We have introduced a cutoff $\Lambda$ in Eq. (61). Since we have only the low-energy part under control where the two-pion state dominates, it is not reasonable to extend the integral into the uncontrolled high-energy region. In practice, the two-pion state dominates the isovector channel up to about $1 \mathrm{GeV}$. To explore the uncertainties of our low-energy approximation, we will vary the cutoff between 1 and $2 \mathrm{GeV}$.

Finally, we come back to the anomalous piece in Eq. (61):

$$
\begin{aligned}
F_{i}^{\text {anom }}\left(q^{2}\right)= & \frac{q^{2}}{12 \pi} \int_{0}^{1} d x \frac{d s^{\prime}(x)}{d x} \frac{1}{s^{\prime}(x)-q^{2}} \\
& \times \frac{f_{i}\left(s^{\prime}(x)\right) F_{\pi}^{V}\left(s^{\prime}(x)\right)}{-4\left[-\lambda\left(s^{\prime}(x), m_{\Sigma^{*}}^{2}, m_{\Lambda}^{2}\right)\right]^{3 / 2}} .
\end{aligned}
$$

The drawback of Eq. (61) is that one needs experimental input for the three complex-valued subtraction constants $F_{i}(0)$. This is on top of the constants $P_{m}$ in Eq. (45), which are ideally also fitted to experimental data. At the moment, such an amount of experimental information is not available. Therefore, we will explore an alternative in the next subsection.

\section{Unsubtracted dispersion relations}

At large energies, the TFFs $F_{i}$ determined via (61) approach a constant, in sharp contrast to the correct scaling behavior (17). The TFFs $G_{m}$ obtained from (8)-(10) even diverge. All this is not a fundamental problem since by construction the representation (61) is designed to be accurate at low energies only. Nonetheless, the representation (61) requires the knowledge of several subtraction constants, all of them in principle complex, because the $\Sigma^{*}$ is unstable. Thus, it might be of advantage to explore the predictive power of an unsubtracted dispersion relation. As shown, e.g., in Refs. [11,16,21], 
one cannot expect to obtain completely correct values for the subtraction constants from the unsubtracted dispersion relations, if one uses only the two-pion intermediate states. However, it might be reasonable to use a simple effective pole to approximate the impact of all the other, higher lying intermediate states on the low-energy quantities [21,46,47]. The pole position might be varied in a reasonable range of masses of excited vector mesons [35] while the residue can be chosen such that a more reasonable high-energy behavior is achieved.

Enforcing a more realistic high-energy behavior provides an additional advantage. As already pointed out, one can then formulate simple dispersion relations also for the TFFs $G_{m}$, $m=0, \pm 1$. In practice, we write

$$
\begin{aligned}
G_{m}\left(q^{2}\right)= & \frac{1}{12 \pi} \int_{4 m_{\pi}^{2}}^{\Lambda^{2}} \frac{d s}{\pi} \frac{T_{m}(s) p_{\mathrm{c} . \mathrm{m} .}^{3}(s) F_{\pi}^{V *}(s)}{s^{1 / 2}\left(s-q^{2}-i \epsilon\right)} \\
& +G_{m}^{\mathrm{anom}}\left(q^{2}\right)+c_{m} \frac{M_{V}^{2}}{M_{V}^{2}-q^{2}},
\end{aligned}
$$

which is only valid for $q^{2} \ll M_{V}^{2}$. The anomalous part is given in Eq. (60). The dimensionless constant $c_{m}$ is adjusted such that

This leads to

$$
\lim _{Q^{2} \rightarrow \infty} Q^{2} G_{m}\left(-Q^{2}\right)=0
$$

$$
\begin{aligned}
c_{m} M_{V}^{2}= & -\frac{1}{12 \pi} \int_{4 m_{\pi}^{2}}^{\Lambda^{2}} \frac{d s}{\pi} \frac{T_{m}(s) p_{\text {c.m. }}^{3}(s) F_{\pi}^{V *}(s)}{s^{1 / 2}} \\
& -\frac{1}{12 \pi} \int_{0}^{1} d x \frac{d s^{\prime}(x)}{d x} \frac{f_{m}\left(s^{\prime}(x)\right) s^{\prime}(x) F_{\pi}^{V}\left(s^{\prime}(x)\right)}{-4\left[-\lambda\left(s^{\prime}(x), m_{\Sigma^{*}}^{2}, m_{\Lambda}^{2}\right)\right]^{3 / 2}} .
\end{aligned}
$$

To explore the uncertainties of this approach, one might vary the effective pole between the masses of the excited vector mesons [35], $1.4 \mathrm{GeV}<M_{V}<1.7 \mathrm{GeV}$.

In practice, comparison to experimental results for $\Sigma^{*} \rightarrow$ $\Lambda \gamma$ and $\Sigma^{*} \rightarrow \Lambda e^{+} e^{-}$must show if (64) and (65) is a reasonable approach or if one has to resort to the subtracted dispersion relations (61). So far, there are no Dalitz decay data available. In Sec. V, we present numerical results utilizing (64) and (66).

\section{INPUT FROM CHIRAL PERTURBATION THEORY}

The leading-order (LO) chiral Lagrangian including the decuplet states is given by [7,22,24,25]

$$
\begin{aligned}
\mathcal{L}_{\text {baryon }}^{(1)}= & \operatorname{tr}\left(\bar{B}\left(i \not D-m_{(8)}\right) B\right) \\
& +\bar{T}_{a b c}^{\mu}\left(i \gamma_{\mu \nu \alpha} D^{\alpha}-\gamma_{\mu \nu} m_{(10)}\right)\left(T^{\nu}\right)^{a b c} \\
& +\frac{D}{2} \operatorname{tr}\left(\bar{B} \gamma^{\mu} \gamma_{5}\left\{u_{\mu}, B\right\}\right)+\frac{F}{2} \operatorname{tr}\left(\bar{B} \gamma^{\mu} \gamma_{5}\left[u_{\mu}, B\right]\right) \\
& +\frac{h_{A}}{2 \sqrt{2}}\left[\epsilon^{a d e} \bar{T}_{a b c}^{\mu}\left(u_{\mu}\right)_{d}^{b} B_{e}^{c}+\epsilon_{a d e} \bar{B}_{c}^{e}\left(u^{\mu}\right)_{b}^{d} T_{\mu}^{a b c}\right] \\
& -\frac{H_{A}}{4 m_{R}} \epsilon_{\mu \nu \alpha \beta}\left[\bar{T}_{a b c}^{\mu}\left(D^{\nu} T^{\alpha}\right)^{a b d}\left(u^{\beta}\right)_{d}^{c}\right. \\
& \left.+\left(D^{\nu} \bar{T}^{\alpha}\right)_{a b d}\left(T^{\mu}\right)^{a b c}\left(u^{\beta}\right)_{c}^{d}\right]
\end{aligned}
$$

with tr denoting a flavor trace.
We have introduced the totally antisymmetrized products of two and three $\gamma$ matrices $^{4}$ [38],

$$
\gamma^{\mu \nu}:=\frac{1}{2}\left[\gamma^{\mu}, \gamma^{\nu}\right]
$$

and

$$
\begin{aligned}
\gamma^{\mu \nu \alpha}:= & \frac{1}{6}\left(\gamma^{\mu} \gamma^{\nu} \gamma^{\alpha}+\gamma^{\nu} \gamma^{\alpha} \gamma^{\mu}+\gamma^{\alpha} \gamma^{\mu} \gamma^{\nu}\right. \\
& \left.-\gamma^{\mu} \gamma^{\alpha} \gamma^{\nu}-\gamma^{\alpha} \gamma^{\nu} \gamma^{\mu}-\gamma^{\nu} \gamma^{\mu} \gamma^{\alpha}\right) \\
= & \frac{1}{2}\left\{\gamma^{\mu \nu}, \gamma^{\alpha}\right\} \\
= & +i \epsilon^{\mu \nu \alpha \beta} \gamma_{\beta} \gamma_{5},
\end{aligned}
$$

respectively. Our conventions are: $\gamma_{5}:=i \gamma^{0} \gamma^{1} \gamma^{2} \gamma^{3}$ and (32), the latter in agreement with Ref. [38] but opposite to Refs. [7,48]. If a formal manipulation program is used to calculate spinor traces and Lorentz contractions, a good check for the convention for the Levi-Civita symbol is the last relation in Eq. (69).

The octet baryons are collected in ( $B_{b}^{a}$ is the entry in the $a$ th row, $b$ th column)

$$
B=\left(\begin{array}{ccc}
\frac{1}{\sqrt{2}} \Sigma^{0}+\frac{1}{\sqrt{6}} \Lambda & \Sigma^{+} & p \\
\Sigma^{-} & -\frac{1}{\sqrt{2}} \Sigma^{0}+\frac{1}{\sqrt{6}} \Lambda & n \\
\Xi^{-} & \Xi^{0} & -\frac{2}{\sqrt{6}} \Lambda
\end{array}\right) .
$$

The decuplet is expressed by a totally symmetric flavor tensor $T^{a b c}$ with

$$
\begin{aligned}
T^{111} & =\Delta^{++}, \quad T^{112}=\frac{1}{\sqrt{3}} \Delta^{+}, \\
T^{122} & =\frac{1}{\sqrt{3}} \Delta^{0}, \quad T^{222}=\Delta^{-}, \\
T^{113} & =\frac{1}{\sqrt{3}} \Sigma^{*+}, \quad T^{123}=\frac{1}{\sqrt{6}} \Sigma^{* 0}, \\
T^{223} & =\frac{1}{\sqrt{3}} \Sigma^{*-}, \quad T^{133}=\frac{1}{\sqrt{3}} \Xi^{* 0}, \\
T^{233} & =\frac{1}{\sqrt{3}} \Xi^{*-}, \quad T^{333}=\Omega .
\end{aligned}
$$

The Goldstone bosons are encoded in

$$
\begin{aligned}
& \Phi=\left(\begin{array}{ccc}
\pi^{0}+\frac{1}{\sqrt{3}} \eta & \sqrt{2} \pi^{+} & \sqrt{2} K^{+} \\
\sqrt{2} \pi^{-} & -\pi^{0}+\frac{1}{\sqrt{3}} \eta & \sqrt{2} K^{0} \\
\sqrt{2} K^{-} & \sqrt{2} \bar{K}^{0} & -\frac{2}{\sqrt{3}} \eta
\end{array}\right), \\
& u^{2}:=U:=\exp \left(i \Phi / F_{\pi}\right), \quad u_{\mu}:=i u^{\dagger}\left(\nabla_{\mu} U\right) u^{\dagger}=u_{\mu}^{\dagger} .
\end{aligned}
$$

The fields have the following transformation properties with respect to chiral transformations [15,22]:

$$
\begin{aligned}
U & \rightarrow L U R^{\dagger}, \\
u & \rightarrow L u h^{\dagger}=h u R^{\dagger}, \\
u_{\mu} & \rightarrow h u_{\mu} h^{\dagger},
\end{aligned}
$$

\footnotetext{
${ }^{4}$ Throughout this work, when using the phrase " $\gamma$ matrices" we have the four $\gamma$ matrices $\gamma^{\mu}, \mu=0,1,2,3$, in mind, not $\gamma_{5}$.
} 


$$
\begin{aligned}
B & \rightarrow h B h^{\dagger}, \\
T_{\mu}^{a b c} & \rightarrow h_{d}^{a} h_{e}^{b} h_{f}^{c} T_{\mu}^{d e f}, \\
\bar{T}_{a b c}^{\mu} & \rightarrow\left(h^{\dagger}\right)_{a}^{d}\left(h^{\dagger}\right)_{b}^{e}\left(h^{\dagger}\right)_{c}^{f} \bar{T}_{d e f}^{\mu} .
\end{aligned}
$$

In particular, the choice of upper and lower flavor indices is used to indicate that upper indices transform with $h$ under flavor transformations while the lower components transform with $h^{\dagger}$.

For a (baryon) octet, the chirally covariant derivatives are defined by

$$
D^{\mu} B:=\partial^{\mu} B+\left[\Gamma^{\mu}, B\right],
$$

for a decuplet $T$ by

$$
\begin{aligned}
\left(D^{\mu} T\right)^{a b c}:= & \partial^{\mu} T^{a b c}+\left(\Gamma^{\mu}\right)_{a^{\prime}}^{a} T^{a^{\prime} b c} \\
& +\left(\Gamma^{\mu}\right)_{b^{\prime}}^{b} T^{a b^{\prime} c}+\left(\Gamma^{\mu}\right)_{c^{\prime}}^{c} T^{a b c^{\prime}},
\end{aligned}
$$

for an antidecuplet by

$$
\begin{aligned}
\left(D^{\mu} \bar{T}\right)_{a b c}:= & \partial^{\mu} \bar{T}_{a b c}-\left(\Gamma^{\mu}\right)_{a}^{a^{\prime}} \bar{T}_{a^{\prime} b c} \\
& -\left(\Gamma^{\mu}\right)_{b}^{b^{\prime}} \bar{T}_{a b^{\prime} c}-\left(\Gamma^{\mu}\right)_{c}^{c^{\prime}} \bar{T}_{a b c^{\prime}},
\end{aligned}
$$

and for the Goldstone boson fields by

$$
\nabla_{\mu} U:=\partial_{\mu} U-i\left(v_{\mu}+a_{\mu}\right) U+i U\left(v_{\mu}-a_{\mu}\right)
$$

with

$\Gamma_{\mu}:=\frac{1}{2}\left[u^{\dagger}\left(\partial_{\mu}-i\left(v_{\mu}+a_{\mu}\right)\right) u+u\left(\partial_{\mu}-i\left(v_{\mu}-a_{\mu}\right)\right) u^{\dagger}\right]$,

where $v$ and $a$ denote external sources.

in Eq. (67), $m_{(8)}\left(m_{(10)}\right)$ denotes the mass of the baryon octet (decuplet) in the chiral limit. For the next-to-leadingorder (NLO) calculation that we perform in the present work, we use the physical masses [35] of all states. Indeed, for the octet and decuplet, the flavor-breaking terms that appear at NLO, cf. (81) and (82) below, are capable of splitting up the baryon masses such that they are sufficiently close to the physical masses; see, e.g., the corresponding discussion in Refs. [9,25].

For the coupling constants, we use $D=0.80, F=0.46$, which implies for the pion-nucleon coupling constant $g_{A}=$ $F+D=1.26$. The value for $h_{A}$ can be determined from the partial decay width $\Sigma^{*} \rightarrow \pi \Lambda$ or $\Sigma^{*} \rightarrow \pi \Sigma$, yielding $h_{A}=$ $2.3 \pm 0.1$ [11]. For a large number of colors, $N_{c}$, one obtains the following relations for two or three flavors, respectively: $h_{A}=3 g_{A} / \sqrt{2} \approx 2.67$ according to Refs. [7,48,49] or $h_{A}=$ $2 \sqrt{2} D \approx 2.26$ according to Refs. [23,50]. Finally, one has to specify $H_{A}$. In the absence of a simple direct observable to pin it down, we take estimates from large- $N_{c}$ considerations: $H_{A}=\frac{9}{5} g_{A} \approx 2.27[7,48]$ or $H_{A}=9 F-3 D \approx 1.74[23,50]$. Numerically, we explore the range $H_{A}=2.0 \pm 0.3$. We have checked explicitly that the sign of $H_{A}$ is in agreement with Refs. [7,48] and also with Ref. [50]. For quark-model estimates of these coupling constants, see Refs. [51,52]. As mentioned previously, the current data situation does not allow for a comparison between results obtained from unsubtracted and subtracted dispersion relations. This implies that it would be premature to conduct a full-fledged error analysis at the moment. We estimate instead how our results are sensitive to variations in the parameters and collect this information in the tables of Sec. V.

For our purposes, the interaction term proportional to $H_{A}$ effectively reduces to

$$
+\frac{H_{A}}{2 m_{R} F_{\pi}} \epsilon_{\mu \nu \alpha \beta} \bar{T}_{a b c}^{\mu} \partial^{\nu}\left(T^{\alpha}\right)^{a b d} \partial^{\beta} \Phi_{d}^{c} .
$$

Working with relativistic spin-3/2 Rarita-Schwinger fields is plagued by ambiguities as to how to deal with the spurious spin-1/2 components. In the present context, the interaction term $\sim h_{A}$ not only causes the proper exchange of spin-3/2 resonances but also induces an additional contact interaction. This unphysical contribution can be avoided by constructing interaction terms according to the Pascalutsa description $[7,48,49,53]$. It boils down to the replacement

$$
T^{\mu} \rightarrow-\frac{1}{m_{R}} \epsilon^{\nu \mu \alpha \beta} \gamma_{5} \gamma_{\nu} \partial_{\alpha} T_{\beta},
$$

where $m_{R}$ denotes the resonance mass. Strictly speaking, this procedure induces an explicit flavor breaking, but such effects are anyway beyond leading order. In practice, we take the mass of the $\Sigma^{*}$ resonance. The $H_{A}$ term of (79) is already constructed such that only the spin- $3 / 2$ components contribute.

We will explore both the standard interaction term $\sim h_{A}$ from (67) and the corresponding one obtained by (80). We will show explicitly that differences can be accounted for by contact interactions of the chiral Lagrangian at NLO and beyond. Quantitatively, it is interesting to see how much the contact terms $P_{m}$ in Eq. (45) are changed when switching from the standard to the Pascalutsa interaction. This provides an uncertainty estimate if $P_{m}$ is not determined from a fit to form factor data. In principle, we could do the same for the $H_{A}$ term and start instead with a simpler Lagrangian $\sim \bar{T}_{a b c}^{\mu}[\mu]_{d}^{c} \gamma_{5} T_{\mu}^{a b d}$, but we refrain from this exercise.

Now we turn to the Lagrangian of second order in the chiral counting. A complete and minimal NLO Lagrangian has been presented in Ref. [25]. For our present purpose, we need terms that lift the mass degeneracies that hold at LO and we need terms that provide interactions for $\Sigma^{*} \pi \rightarrow \Lambda \pi$ (or formally $\Sigma^{*} \bar{\Lambda} \rightarrow 2 \pi$ ) with the two pions in a $p$ wave.

The relevant part of the NLO Lagrangian for the baryon octet sector reads $[25,54,55]$

$$
\mathcal{L}_{8}^{(2)}=b_{\chi, D} \operatorname{tr}\left(\bar{B}\left\{\chi_{+}, B\right\}\right)+b_{\chi, F} \operatorname{tr}\left(\bar{B}\left[\chi_{+}, B\right]\right)
$$

with $\chi_{ \pm}=u^{\dagger} \chi u^{\dagger} \pm u \chi^{\dagger} u$ and $\chi=2 B_{0}(s+i p)$ obtained from the scalar source $s$ and the pseudoscalar source $p$. The low-energy constant $B_{0}$ is essentially the ratio of the lightquark condensate and the square of the pion-decay constant; see, e.g., Refs. [15,56-58]. While at LO all baryon octet states are degenerate in mass, the NLO terms of (81) lift this degeneracy and essentially move all masses to their respective physical values. Technically, this is achieved if one replaces the scalar source $s$ by the quark mass matrix. Numerical results for the octet mass $m_{(8)}$ in Eq. (67) and the splitting parameters $b_{\chi, D / \chi, F}$ in Eq. (81) are given, for instance, in Ref. [9]. In practice, we use the physical masses. Therefore, we do not specify these parameters here. 
The relevant part of the NLO Lagrangian for the baryon decuplet sector reads [25]

$$
\mathcal{L}_{10}^{(2)}=-d_{\chi,(8)} \bar{T}_{a b c}^{\mu}\left(\chi_{+}\right)_{d}^{c} \gamma_{\mu \nu}\left(T^{\nu}\right)^{a b d} .
$$

It provides a mass splitting for the decuplet baryons such that $m_{\Omega}-m_{\Xi^{*}}=m_{\Xi^{*}}-m_{\Sigma^{*}}=m_{\Sigma^{*}}-m_{\Delta}$, in good agreement with phenomenology [35]. In the present work, we only deal with the $\Sigma^{*}$. In practice, we use the physical mass of the neutral $\Sigma^{*}$. In that way the physical thresholds are exactly reproduced.

More concretely, we use the following masses (in GeV): $m_{\pi}=0.13957, m_{\Lambda}=1.116, m_{\Sigma}=1.193$, and $m_{\Sigma^{*}}=1.384$.

For the formal reaction $\Sigma^{*} \bar{\Lambda} \rightarrow 2 \pi$, the relevant part of the NLO Lagrangian [25] is given by

$$
\mathcal{L}_{8-10}^{(2)} \rightarrow \frac{c_{F}}{2 F_{\pi}^{2}} \bar{\Lambda} \gamma_{\mu} \gamma_{5} \Sigma_{\nu}^{* 0}\left(\partial^{\mu} \pi^{+} \partial^{\nu} \pi^{-}-(\mu \leftrightarrow v)\right)
$$

A vector-meson-dominance estimate for $c_{F}$ is provided in Appendix D.

\section{RESULTS}

\section{A. Matrix elements}

The first step is the calculation of the pion-hyperon tree-level amplitudes, i.e., $\chi \mathrm{PT}$ amplitudes up to (including) NLO. In practice, the extraction of the reduced amplitudes is simplified and systemized by a projector formalism presented in Appendix B. Note that these amplitudes constitute the leading, i.e., dominant contribution; the notation NLO refers to the underlying chiral Lagrangian, whose tree-level contribution is equally important to that coming from the LO Lagrangian.

The Feynman matrix element for the reaction $\Sigma^{* 0} \bar{\Lambda} \rightarrow \pi^{+} \pi^{-}$up to (including) NLO is given by

$$
\begin{aligned}
& -\frac{D h_{A}}{6 \sqrt{2} F_{\pi}^{2}} \frac{1}{t-m_{\Sigma}^{2}+i \epsilon} p_{\pi^{+}}^{\mu} g_{\mu \alpha} \bar{v}_{\Lambda} \not p_{\pi^{-}} \gamma_{5}\left(\not p_{\Sigma^{*}}-\not p_{\pi^{+}}+m_{\Sigma}\right) u_{\Sigma^{*}}^{\alpha} \\
& \quad+\frac{D h_{A}}{6 \sqrt{2} F_{\pi}^{2}} \frac{1}{u-m_{\Sigma}^{2}+i \epsilon} p_{\pi^{-}}^{\mu} g_{\mu \alpha} \bar{v}_{\Lambda} \not p_{\pi^{+}} \gamma_{5}\left(\not p_{\Sigma^{*}}-\not p_{\pi^{-}}+m_{\Sigma}\right) u_{\Sigma^{*}}^{\alpha}+\frac{h_{A} H_{A}}{6 \sqrt{2} m_{\Sigma^{*}} F_{\pi}^{2}} i \epsilon_{\nu \alpha \beta}^{\lambda} p_{\Sigma^{*}}^{\nu} p_{\pi^{+}}^{\beta} p_{\pi^{-}}^{\mu} \bar{v}_{\Lambda} S_{\mu \lambda}\left(p_{\Sigma^{*}}-p_{\pi^{+}}\right) u_{\Sigma^{*}}^{\alpha} \\
& \quad-\frac{h_{A} H_{A}}{6 \sqrt{2} m_{\Sigma^{*}} F_{\pi}^{2}} i \epsilon_{\nu \alpha \beta}^{\lambda} p_{\Sigma^{*}}^{v} p_{\pi^{-}}^{\beta} p_{\pi^{+}}^{\mu} \bar{v}_{\Lambda} S_{\mu \lambda}\left(p_{\Sigma^{*}}-p_{\pi^{-}}\right) u_{\Sigma^{*}}^{\alpha}+\frac{c_{F}}{2 F_{\pi}^{2}}\left(p_{\pi^{+}}^{\mu} p_{\pi^{-}}^{\alpha}-p_{\pi^{+}}^{\alpha} p_{\pi^{-}}^{\mu}\right) g_{\alpha \beta} \bar{v}_{\Lambda} \gamma_{\mu} \gamma_{5} u_{\Sigma^{*}}^{\beta} .
\end{aligned}
$$

Here $S_{\mu \nu}$ denotes the spin-3/2 propagator given in Eq. (B7).

Using FeynCalc [59,60], we obtain the following amplitudes from the $\Sigma$ and $\Sigma^{*}$ exchange diagrams:

with

$$
\begin{aligned}
K_{+1} & =\frac{D h_{A}}{6 \sqrt{2} F_{\pi}^{2}}\left(C_{+1}+D_{+1} R_{s}^{\text {oct. }}\right)+\frac{h_{A} H_{A}}{6 \sqrt{2} F_{\pi}^{2}}\left(E_{+1}+F_{+1} R_{s}^{\text {dec. }}\right), \\
K_{-1} & =\frac{D h_{A}}{6 \sqrt{2} F_{\pi}^{2}}\left(C_{-1}+D_{-1} R_{s}^{\text {oct. }}\right)+\frac{h_{A} H_{A}}{6 \sqrt{2} F_{\pi}^{2}}\left(E_{-1}+F_{-1} R_{s}^{\text {dec. }}\right), \\
K_{0} & =\frac{D h_{A}}{6 \sqrt{2} F_{\pi}^{2}}\left(C_{0}+D_{0} R_{d}^{\text {oct. }}\right)+\frac{h_{A} H_{A}}{6 \sqrt{2} F_{\pi}^{2}}\left(E_{0}+F_{0} R_{d}^{\text {dec. }}\right)
\end{aligned}
$$

and

$$
\begin{aligned}
& R_{s}^{\text {oct. }}=\frac{-2 Y_{\Sigma}}{\kappa^{2}}\left\{1-\left(1-\frac{Y_{\Sigma}^{2}}{\kappa^{2}}\right) \frac{|\kappa|}{Y_{\Sigma}}\left[\arctan \left(\frac{|\kappa|}{Y_{\Sigma}}\right)+\pi \Theta\left(s_{3}-s\right)\right]\right\} \\
& R_{d}^{\text {oct. }}=\frac{4}{\kappa^{2}}\left\{1-\frac{Y_{\Sigma}}{|\kappa|}\left[\arctan \left(\frac{|\kappa|}{Y_{\Sigma}}\right)+\pi \Theta\left(s_{3}-s\right)\right]\right\}, \\
& R_{s}^{\text {dec. }}=\frac{-2 Y_{\Sigma^{*}}}{\kappa^{2}}\left[1-\left(1-\frac{Y_{\Sigma^{*}}^{2}}{\kappa^{2}}\right) \frac{|\kappa|}{Y_{\Sigma^{*}}} \arctan \left(\frac{|\kappa|}{Y_{\Sigma^{*}}}\right)\right], \\
& R_{d}^{\text {dec. }}=\frac{4}{\kappa^{2}}\left[1-\frac{Y_{\Sigma^{*}}}{|\kappa|} \arctan \left(\frac{|\kappa|}{Y_{\Sigma^{*}}}\right)\right]
\end{aligned}
$$

$$
\begin{aligned}
Y_{\Sigma} & =2 m_{\Sigma^{2}}^{2}-m_{\Sigma^{*}}^{2}-m_{\Lambda}^{2}-2 m_{\pi}^{2}+s, \\
Y_{\Sigma^{*}} & =m_{\Sigma^{*}}^{2}-m_{\Lambda}^{2}-2 m_{\pi}^{2}+s, \\
\kappa^{2} & =\frac{1}{s}\left(s-4 m_{\pi}^{2}\right) \lambda\left(s, m_{\Sigma^{*}}^{2}, m_{\Lambda}^{2}\right), \\
s_{3} & =m_{\Sigma^{*}}^{2}+m_{\Lambda}^{2}+2 m_{\pi}^{2}-2 m_{\Sigma^{2}}^{2} .
\end{aligned}
$$


Note that $\kappa^{2}$ is negative in the range $4 m_{\pi}^{2}<s<\left(m_{\Sigma^{*}}+m_{\Lambda}\right)^{2}$, i.e. $|\kappa|=\sqrt{-\kappa^{2}}$. Only for negative $\kappa^{2}$ the expressions (86) are correct. For positive $\kappa^{2}$ one has log's instead of arctan's.

Finally, the coefficient functions in Eq. (85) are given by

$$
\begin{aligned}
& C_{+1}=-\frac{2\left(m_{\Sigma^{*}}-m_{\Lambda}\right)\left(m_{\Lambda}+m_{\Sigma}\right)}{s-\left(m_{\Sigma^{*}}-m_{\Lambda}\right)^{2}}, \\
& C_{-1}=-\frac{6\left(m_{\Sigma^{*}}-m_{\Lambda}\right)\left(m_{\Lambda}+m_{\Sigma}\right)}{s-\left(m_{\Sigma^{*}}-m_{\Lambda}\right)^{2}}, \\
& C_{0}=\frac{\left(m_{\Sigma^{*}}+m_{\Lambda}\right)\left(m_{\Sigma^{*}}+m_{\Sigma}\right)}{s}-\frac{3 m_{\Sigma^{*}}\left(m_{\Lambda}+m_{\Sigma}\right)}{s-\left(m_{\Sigma^{*}}-m_{\Lambda}\right)^{2}}, \\
& D_{+1}=3 m_{\Sigma}\left(m_{\Lambda}+m_{\Sigma}\right)+\frac{3\left(m_{\Sigma^{*}}-m_{\Lambda}\right)\left(m_{\Lambda}+m_{\Sigma}\right)\left(m_{\pi}^{2}+m_{\Sigma^{*}} m_{\Lambda}-m_{\Sigma}^{2}\right)}{s-\left(m_{\Sigma^{*}}-m_{\Lambda}\right)^{2}}, \\
& D_{-1}=\frac{3}{m_{\Sigma^{*}}}\left(m_{\Lambda}+m_{\Sigma}\right)\left(m_{\pi}^{2}-m_{\Sigma^{*}}^{2}+m_{\Sigma^{*}} m_{\Sigma}-m_{\Sigma}^{2}\right)+\frac{9\left(m_{\Sigma^{*}}-m_{\Lambda}\right)\left(m_{\Lambda}+m_{\Sigma}\right)\left(m_{\pi}^{2}+m_{\Sigma^{*}} m_{\Lambda}-m_{\Sigma}^{2}\right)}{s-\left(m_{\Sigma^{*}}-m_{\Lambda}\right)^{2}}, \\
& D_{0}=3 m_{\Sigma}\left(m_{\Lambda}+m_{\Sigma}\right)\left(m_{\Sigma^{*}}^{2}-m_{\Sigma^{*}} m_{\Sigma}-m_{\pi}^{2}+m_{\Sigma}^{2}\right)-\frac{9 m_{\Sigma^{*}}\left(m_{\Lambda}+m_{\Sigma}\right)\left(m_{\Sigma^{*}} m_{\Lambda}+m_{\pi}^{2}-m_{\Sigma}^{2}\right)^{2}}{s-\left(m_{\Sigma^{*}}-m_{\Lambda}\right)^{2}} \\
& +\frac{3\left(m_{\Sigma^{*}}+m_{\Lambda}\right)\left(m_{\Sigma}+m_{\Lambda}\right)}{s}\left[m_{\Sigma^{*}}^{3} m_{\Lambda}-m_{\Sigma}\left(m_{\Sigma^{*}}-m_{\Lambda}\right)\left(m_{\Sigma^{*}}^{2}+m_{\pi}^{2}\right)+2 m_{\Sigma^{*}}^{2} m_{\pi}^{2}\right. \\
& \left.-m_{\Sigma}^{2}\left(m_{\Sigma^{*}}\left(m_{\Sigma^{*}}+m_{\Lambda}\right)+2 m_{\pi}^{2}\right)+2 m_{\Sigma^{*}} m_{\Lambda} m_{\pi}^{2}-m_{\Sigma}^{3}\left(m_{\Lambda}-m_{\Sigma^{*}}\right)+m_{\pi}^{4}+m_{\Sigma}^{4}\right], \\
& E_{+1}=\frac{\left(m_{\Sigma^{*}}-m_{\Lambda}\right)\left[\left(m_{\Sigma^{*}}+m_{\Lambda}\right)^{2}-m_{\pi}^{2}\right]}{3 m_{\Sigma^{*}}\left[s-\left(m_{\Sigma^{*}}-m_{\Lambda}\right)^{2}\right]}, \\
& E_{-1}=\frac{\left(m_{\Sigma^{*}}-m_{\Lambda}\right)\left[\left(m_{\Sigma^{*}}+m_{\Lambda}\right)^{2}-m_{\pi}^{2}\right]}{m_{\Sigma^{*}}\left[s-\left(m_{\Sigma^{*}}-m_{\Lambda}\right)^{2}\right]}, \\
& E_{0}=-\frac{\left(m_{\Sigma^{*}}+m_{\Lambda}\right)\left(2 m_{\Sigma^{*}}^{2}+2 m_{\Sigma^{*}} m_{\Lambda}-m_{\pi}^{2}\right)}{6 m_{\Sigma^{*}} S}+\frac{\left(m_{\Sigma^{*}}+m_{\Lambda}\right)^{2}-m_{\pi}^{2}}{2\left[s-\left(m_{\Sigma^{*}}-m_{\Lambda}\right)^{2}\right]}, \\
& F_{+1}=-\frac{3 s}{2}-\frac{m_{\pi}^{2}\left(2 m_{\Sigma^{*}}+3 m_{\Lambda}\right)}{2 m_{\Sigma^{*}}}+\frac{5\left(m_{\Sigma^{*}}+m_{\Lambda}\right)^{2}}{2}+\frac{\left(m_{\Sigma^{*}}-m_{\Lambda}\right)\left[\left(m_{\Sigma^{*}}+m_{\Lambda}\right)^{2}-m_{\pi}^{2}\right]\left(m_{\Sigma^{*}}^{2}-m_{\Sigma^{*}} m_{\Lambda}-m_{\pi}^{2}\right)}{2 m_{\Sigma^{*}}\left[s-\left(m_{\Sigma^{*}}-m_{\Lambda}\right)^{2}\right]}, \\
& F_{-1}=\frac{3 s}{2}+\frac{m_{\pi}^{2}\left(m_{\Sigma^{*}}^{2}+m_{\Sigma^{*}} m_{\Lambda}-m_{\Lambda}^{2}\right)+m_{\pi}^{4}}{2 m_{\Sigma^{*}}^{2}}-\frac{5\left(m_{\Sigma^{*}}+m_{\Lambda}\right)^{2}}{2} \\
& +\frac{3\left(m_{\Sigma^{*}}-m_{\Lambda}\right)\left[\left(m_{\Sigma^{*}}+m_{\Lambda}\right)^{2}-m_{\pi}^{2}\right]\left(m_{\Sigma^{*}}^{2}-m_{\Sigma^{*}} m_{\Lambda}-m_{\pi}^{2}\right)}{2 m_{\Sigma^{*}}\left[s-\left(m_{\Sigma^{*}}-m_{\Lambda}\right)^{2}\right]}, \\
& F_{0}=\frac{3 m_{\Sigma^{*}}^{2} s}{2}-\frac{m_{\pi}^{2}\left(7 m_{\Sigma^{*}}^{2}-2 m_{\Sigma^{*}} m_{\Lambda}+2 m_{\Lambda}^{2}\right)+m_{\Sigma^{*}}^{2}\left(m_{\Sigma^{*}}+m_{\Lambda}\right)^{2}}{2}+m_{\pi}^{4} \\
& +\frac{4 m_{\Sigma^{*}}^{2} m_{\pi}^{2}\left(m_{\Sigma^{*}}-2 m_{\Lambda}\right)\left(m_{\Sigma^{*}}+m_{\Lambda}\right)^{2}-m_{\pi}^{4}\left(2 m_{\Sigma^{*}}^{3}+m_{\Sigma^{*}}^{2} m_{\Lambda}+m_{\Lambda}^{3}\right)+m_{\pi}^{6}\left(m_{\Sigma^{*}}+m_{\Lambda}\right)}{2 m_{\Sigma^{*}} S} \\
& +\frac{3\left[\left(m_{\Sigma^{*}}+m_{\Lambda}\right)^{2}-m_{\pi}^{2}\right]\left[m_{\Sigma^{*}}\left(m_{\Lambda}-m_{\Sigma^{*}}\right)+m_{\pi}^{2}\right]^{2}}{2\left[s-\left(m_{\Sigma^{*}}-m_{\Lambda}\right)^{2}\right]} .
\end{aligned}
$$

The explicit expressions for the polynomial terms are

$$
\begin{aligned}
P_{+1} & =\frac{D h_{A}}{6 \sqrt{2} F_{\pi}^{2}} 2+\frac{h_{A} H_{A}}{6 \sqrt{2} F_{\pi}^{2}} \frac{5\left(m_{\Sigma^{*}}+m_{\Lambda}\right)}{6 m_{\Sigma^{*}}}, \\
P_{-1} & =\frac{D h_{A}}{6 \sqrt{2} F_{\pi}^{2}} \frac{2\left(m_{\Sigma^{*}}-m_{\Lambda}-m_{\Sigma}\right)}{m_{\Sigma^{*}}}+\frac{h_{A} H_{A}}{6 \sqrt{2} F_{\pi}^{2}} \frac{s-2 m_{\pi}^{2}-\left(m_{\Sigma^{*}}+m_{\Lambda}\right)\left(6 m_{\Sigma^{*}}-m_{\Lambda}\right)}{6 m_{\Sigma^{*}}^{2}} \\
& \approx \frac{D h_{A}}{6 \sqrt{2} F_{\pi}^{2}} \frac{2\left(m_{\Sigma^{*}}-m_{\Lambda}-m_{\Sigma}\right)}{m_{\Sigma^{*}}}+\frac{h_{A} H_{A}}{6 \sqrt{2} F_{\pi}^{2}} \frac{-\left(m_{\Sigma^{*}}+m_{\Lambda}\right)\left(6 m_{\Sigma^{*}}-m_{\Lambda}\right)}{6 m_{\Sigma^{*}}^{2}}, \\
P_{0} & =\frac{D h_{A}}{6 \sqrt{2} F_{\pi}^{2}}+\frac{h_{A} H_{A}}{6 \sqrt{2} F_{\pi}^{2}} \frac{3 m_{\Sigma^{*}}-m_{\Lambda}}{6 m_{\Sigma^{*}}} .
\end{aligned}
$$


For $P_{-1}$ we dropped terms which are suppressed by two orders in the chiral counting.

The $\Sigma^{*} \Lambda \pi^{+} \pi^{-}$contact diagram produces the following polynomials:

$$
\begin{aligned}
P_{+1}^{\mathrm{NLO} \chi \mathrm{PT}} & =c_{F} \frac{m_{\Sigma^{*}}+m_{\Lambda}}{2 F_{\pi}^{2}}, \\
P_{0}^{\mathrm{NLO} \chi \mathrm{PT}} & =c_{F} \frac{m_{\Sigma^{*}}}{2 F_{\pi}^{2}}, \\
P_{-1}^{\mathrm{NLO} \chi \mathrm{PT}} & =c_{F} \frac{s-m_{\Lambda}\left(m_{\Sigma^{*}}+m_{\Lambda}\right)}{2 F_{\pi}^{2} m_{\Sigma^{*}}} \\
& \approx-c_{F} \frac{m_{\Lambda}\left(m_{\Sigma^{*}}+m_{\Lambda}\right)}{2 F_{\pi}^{2} m_{\Sigma^{*}}} .
\end{aligned}
$$

At this point, it is worth commenting on the power counting, i.e., on the relative importance of contributions from the LO and from the NLO Lagrangian. In principle, the succession in baryonic $\chi \mathrm{PT}$ is such that the LO Lagrangian produces reaction amplitudes of order $\mathcal{O}(p)$, where $p$ denotes a generic momentum. The NLO Lagrangian produces reaction amplitudes of order $\mathcal{O}\left(p^{2}\right)$. Each new Lagrangian is down by one power of momentum. However, the partial-wave projected amplitudes provide a twist to this reasoning. They are analytic functions in $s$, not in $\sqrt{s}$. Thus, suppressed contributions are down by at least two powers of momenta. This has important consequences for the importance of contributions from the $\mathrm{LO}$ and NLO Lagrangians.

As expected, the Feynman amplitudes of hyperon-pion scattering emerging from the NLO Lagrangian are of order $\mathcal{O}\left(p^{2}\right)$. The proper partial-wave projection and rescaling introduced in Eq. (44) takes away two powers of momenta. The reduced amplitudes are then of order $\mathcal{O}\left(p^{0}\right)$, i.e., they are constants, and this is exactly what we see in the previous equations for all three helicity amplitudes.

The Feynman amplitudes of hyperon-pion scattering emerging from the LO Lagrangian are at most of order $\mathcal{O}(p)$. The reduced amplitudes are then at most of order $\mathcal{O}\left(p^{-1}\right)$. But this is not possible for analytic functions in $s$. Therefore, also the helicity amplitudes emerging from the LO Lagrangian are of order $\mathcal{O}\left(p^{0}\right)$. They have the same importance as the corresponding amplitudes emerging from the NLO Lagrangian. This actually resembles the situation found already in Ref. [11] in the magnetic sector.

As a consequence of this finding, it turns out that it does not make sense to compare a calculation stemming solely from the LO Lagrangian to an LO + NLO calculation. The labels of the Lagrangians are somewhat misleading when it comes to the helicity amplitudes. Yet, we have to stress that this does not mean that the labels of the Lagrangians are completely useless. Quite the contrary, the next-to-next-to-leading-order (NNLO) Lagrangian yields only subleading contributions, as its name suggests. The Feynman amplitudes are at most of order $\mathcal{O}\left(p^{3}\right)$. The reduced amplitudes are then at most of order $\mathcal{O}(p)$. But this is again not possible (analyticity in $s$ ). Thus, the reduced amplitudes emerging from the NNLO Lagrangian are at most of order $\mathcal{O}\left(p^{2}\right)$, i.e., suppressed relative to the contributions from the LO and NLO Lagrangians that we take into account in the present work.
Here we will not calculate tree-level hyperon-pion scattering amplitudes based on the NNLO Lagrangian and the corresponding loops based on the LO Lagrangian. Our present input for the dispersion relations-tree-level hyperon-pion amplitudes from LO and NLO_corresponds already to a oneloop calculation in $\chi$ PT for the TFFs, additionally improved by the full pion scattering phase shift, which accounts for the physics of the $\rho$ meson. For the present work, we stick to this level of sophistication. Unfortunately, this implies that we cannot offer chiral corrections to our results, because the contributions from the LO and NLO Lagrangians are both dominant contributions. The absence of the possibility to compare dominant and subdominant chiral contributions implies also that one cannot point out very well for which photon virtuality the whole expansion does not work any more. But it should be clear that one should stay sufficiently lower than the $1-\mathrm{GeV}$ scale given by $4 \pi F_{\pi}$ and the typical hadron masses.

The amplitudes (84) become slightly different when the Pascalutsa prescription is used: New contact terms pop up but the pole terms and therefore (85) are not affected. In particular, we have

$$
\begin{aligned}
P_{+1}^{P} & =P_{+1}+\frac{h_{A} H_{A}}{18 \sqrt{2} F_{\pi}^{2} m_{\Sigma^{*}}^{2}}\left[\left(m_{\Lambda}+m_{\Sigma^{*}}\right)\left(2 m_{\Sigma^{*}}+3 m_{\Lambda}\right)-3 s\right], \\
P_{0}^{P} & =P_{0}-\frac{h_{A} H_{A}}{18 \sqrt{2} F_{\pi}^{2}}, \\
P_{-1}^{P} & =P_{-1}-\frac{h_{A} H_{A}}{18 \sqrt{2} F_{\pi}^{2} m_{\Sigma^{*}}^{2}}\left[\left(m_{\Lambda}+m_{\Sigma^{*}}\right)\left(3 m_{\Sigma^{*}}+2 m_{\Lambda}\right)-2 s\right] .
\end{aligned}
$$

As expected, the $\Sigma$ exchange diagrams do not get any contribution since the external $\Sigma^{*}$ hyperon is on shell.

It is illuminating to translate these contact interactions to the $i=1,2,3$ amplitudes defined in Eq. (62). One obtains

$$
P_{1}^{\mathrm{NLO} \chi \mathrm{PT}}=\frac{c_{F}}{2 m_{\Sigma^{*}} F_{\pi}^{2}}, \quad P_{2,3}^{\mathrm{NLO} \chi \mathrm{PT}}=0
$$

and

$$
\begin{aligned}
& P_{1}^{P}=P_{1}+5 \frac{h_{A} H_{A}}{18 \sqrt{2} F_{\pi}^{2} m_{\Sigma^{*}}^{2}}, \\
& P_{2}^{P}=P_{2}-6 \frac{h_{A} H_{A}}{18 \sqrt{2} F_{\pi}^{2} m_{\Sigma^{*}}^{2}}, \\
& P_{3}^{P}=P_{3} .
\end{aligned}
$$

Thus, the NLO contact term can be used to compensate for the difference between naive and Pascalutsa interaction concerning the $i=1$ amplitude structure, but not for $i=2$. In fact, there is a one-to-one correspondence between the contact terms of the pion-hyperon scattering amplitudes and the constraint-free TFFs introduced at the very beginning in Eq. (2). Chiral power counting shows that in $\chi \mathrm{PT}$, the TFF $F_{i}$ receives tree-level contributions starting at chiral order $i+1$. At an NLO accuracy, one has only full access to $F_{1}$. Correspondingly, the NLO contact interaction for the pionhyperon amplitudes contributes only to $P_{1}$ as shown explicitly 
TABLE I. Comparison of the results for various observables using $t_{\mathrm{IAM}}, c_{F}=-6.33 \mathrm{GeV}^{-1}$, central values for $h_{A}, H_{A}$, and $M_{V}$ and varying the cutoff $\Lambda$.

\begin{tabular}{lcc}
\hline \hline Quantity & $\Lambda=1 \mathrm{GeV}$ & $\Lambda=2 \mathrm{GeV}$ \\
\hline$G_{0}(0)$ & $-3.5-0.0 i$ & $-3.7-0.0 i$ \\
$\left\langle r_{0}^{2}\right\rangle\left[\mathrm{GeV}^{-2}\right]$ & $21.5+7.1 i$ & $21.0+6.8 i$ \\
$G_{+1}(0)$ & $-4.5-0.0 i$ & $-4.8-0.0 i$ \\
$\left\langle r_{+1}^{2}\right\rangle\left[\mathrm{GeV}^{-2}\right]$ & $16.9+1.3 i$ & $16.7+1.3 i$ \\
$G_{-1}(0)$ & $3.2-0.0 i$ & $3.5-0.0 i$ \\
$\left\langle r_{-1}^{2}\right\rangle\left[\mathrm{GeV}^{-2}\right]$ & $16.8-1.2 i$ & $16.5-1.2 i$ \\
$\Gamma_{\Sigma^{*} \rightarrow \Lambda e^{+} e^{-}}[\mathrm{keV}]$ & 3.0 & 3.4 \\
$\Gamma_{\Sigma^{*} \rightarrow \Lambda \gamma}[\mathrm{MeV}]$ & 0.39 & 0.45 \\
\hline \hline
\end{tabular}

in Eq. (106). To compensate the difference between naive and Pascalutsa interaction concerning the $i=2$ amplitude structure, one needs a contact term from the next-to-next-toleading-order Lagrangian. This is beyond the scope of the present work.

\section{B. Numerical results}

The results below have been obtained using Pascalutsa amplitudes. They consist in unsubtracted dispersion relations for the TFFs $G_{m}(64)$, evaluated at the photon point $\left(q^{2}=0\right)$, followed by the corresponding radii:

$$
\left\langle r_{m}^{2}\right\rangle:=\left.\frac{6}{G_{m}(0)} \frac{d G_{m}\left(q^{2}\right)}{d q^{2}}\right|_{q^{2}=0} .
$$

Other quantities of interest are the integrated decay rate for $\Sigma^{*} \rightarrow \Lambda e^{+} e^{-}$and the decay width for $\Sigma^{*} \rightarrow \Lambda \gamma$.

We start by fixing the input parameters $h_{A}, H_{A}$, and $M_{V}$ to the respective central value. We will explore later the impact of their uncertainties on the final results, while we will not vary $D$ nor the pion phase shift since they are better constrained. We also want to investigate the dependence on the cutoff $\Lambda$, which we will take equal to 1 and $2 \mathrm{GeV}$, respectively. Furthermore, recall that in order to account for the anomalous contribution (53) to the scattering amplitudes, one needs to know the pion scattering amplitude $t(s)$ in the complex plane. We will explore two options: an approximation from $\chi \mathrm{PT}$ (55), denoted by $t_{\chi \mathrm{PT}}$, and its unitarized version $(56), t_{\mathrm{IAM}}$.

Our strategy is to adjust the dimensionless constants $c_{m}$ 's according to (66) and fix the NLO low-energy constant $c_{F}$ to the experimental value of the decay width $\Sigma^{*} \rightarrow \Lambda \gamma$ which is $(0.45 \pm 0.05) \mathrm{MeV}$ [35]. When matching to the central value $(0.45 \mathrm{MeV})$, one finds two possible solutions: $c_{F}=-6.33 \mathrm{GeV}^{-1}$ and $c_{F}=2.39 \mathrm{GeV}^{-1}$. We pick the first, ${ }^{5}$ being closer to the VMD estimate (D4). The chosen value of $c_{F}$ is kept unchanged throughout the whole analysis, while the constants $c_{m}$ 's are adjusted by (66) each time any other parameter is varied. As explained in Sec. IV, not all sources

\footnotetext{
${ }^{5}$ The results corresponding to the other choice for $c_{F}$ reflect the fact that the two possible values have opposite signs. Otherwise, the results are qualitatively similar.
}

TABLE II. Same as Table I for the comparison between $t_{\chi \mathrm{PT}}$ and $t_{\text {IAM }}$ using $\Lambda=2 \mathrm{GeV}, c_{F}=-6.33 \mathrm{GeV}^{-1}$, central values for $h_{A}$, $H_{A}$, and $M_{V}$.

\begin{tabular}{lcc}
\hline \hline Quantity & \multicolumn{1}{c}{$t_{\chi \mathrm{PT}}$} & $t_{\mathrm{IAM}}$ \\
\hline$G_{0}(0)$ & $-3.7-0.0 i$ & $-3.7-0.0 i$ \\
$\left\langle r_{0}^{2}\right\rangle\left[\mathrm{GeV}^{-2}\right]$ & $20.8+6.7 i$ & $21.0+6.8 i$ \\
$G_{+1}(0)$ & $-4.8-0.0 i$ & $-4.8-0.0 i$ \\
$\left\langle r_{+1}^{2}\right\rangle\left[\mathrm{GeV}^{-2}\right]$ & $16.7+1.3 i$ & $16.7+1.3 i$ \\
$G_{-1}(0)$ & $3.5-0.0 i$ & $3.5-0.0 i$ \\
$\left\langle r_{-1}^{2}\right\rangle\left[\mathrm{GeV}^{-2}\right]$ & $16.5-1.2 i$ & $16.5-1.2 i$ \\
$\Gamma_{\Sigma^{*} \rightarrow \Lambda e^{+} e^{-}}[\mathrm{keV}]$ & 3.4 & 3.4 \\
$\Gamma_{\Sigma^{*} \rightarrow \Lambda \gamma}[\mathrm{MeV}]$ & 0.45 & 0.45 \\
\hline \hline
\end{tabular}

of uncertainties are at present under control, in particular due to the scarce data situation. Therefore, we refrain from taking into account additional uncertainties coming from $c_{F}$. When enough data will allow for an experimental determination of $c_{F}$ (and corresponding error), there will be no need to fix it to the partial decay width. For completeness, we report the $c_{m}$ values obtained when choosing $\Lambda=2 \mathrm{GeV}, t_{\mathrm{IAM}}$, central values for $h_{A}, H_{A}$, and $M_{V}: c_{-1}=-0.59-0.04 i, c_{0}=$ $1.05-0.10 i, c_{+1}=0.96-0.05 i$. This scenario gives rise to the results of Table I, right column.

From Table I, we get the encouraging message that varying the cutoff has a rather small impact. In Table II, we compare the choice of using $t_{\chi \mathrm{PT}}$ versus $t_{\mathrm{IAM}}$. As expected, both approaches lead essentially to the same results. Finally, we study the changes of the $G_{m}(0)$ 's, the radii, the partial widths $\Gamma_{\Sigma^{*} \rightarrow \Lambda e^{+} e^{-}}$and $\Gamma_{\Sigma^{*} \rightarrow \Lambda \gamma}$ when varying $h_{A}, H_{A}$, and $M_{V}$, one at a time, as shown in Table III. The uncertainties related to $h_{A}, H_{A}$, and $M_{V}$ turn out to be moderate and comparable. It is satisfying to observe that the $G_{m}(0)$ values are not subject to large changes and the radii are even less sensitive to these variations. In fact, the dispersive machinery is supposed to work better for the radii since by definition they receive a suppressed contribution from the high-energy region.

The scarce amount of data does not allow for a better estimation of the uncertainties, a crucial task that is left for the future. Tables I-III are meant to cope with this temporary deficiency, showing how the results are affected by broad variations in some of the parameters.

As previously stated, we stick to Pascalutsa amplitudes here, but the very same analysis can be carried out using the naive couplings instead. Note that it would then be necessary to refit $c_{F}$ since the meaning of the contact interaction changes based on which three-point coupling is used. ${ }^{6}$

Still using $t_{\mathrm{IAM}}$, central values for $h_{A}, H_{A}$, and $M_{V}$ and cutoff $\Lambda=2 \mathrm{GeV}$, we plot the real and imaginary part of the TFFs $G_{m}\left(q^{2}\right)$ (64) in the space- and timelike regions, up to $q^{2}=\left(m_{\Sigma^{*}}-m_{\Lambda}\right)^{2}$. As shown in Figs. 1-3, all three functions are complex, already below the two-pion threshold. Technically, this is a consequence of the additional anomalous

\footnotetext{
${ }^{6}$ Again, the final results show similar qualitative behavior as in the Pascalutsa case.
} 
TABLE III. Same as Table I using $t_{\mathrm{IAM}}, \Lambda=2 \mathrm{GeV}, c_{F}=-6.33 \mathrm{GeV}^{-1}$ and varying $h_{A}, H_{A}$, and $M_{V}$ one at a time.

\begin{tabular}{|c|c|c|c|c|c|c|}
\hline Quantity & $h_{A}=2.2$ & $h_{A}=2.4$ & $H_{A}=1.7$ & $H_{A}=2.3$ & $M_{V}=1.4 \mathrm{GeV}$ & $M_{V}=1.7 \mathrm{GeV}$ \\
\hline$\left\langle r_{0}^{2}\right\rangle\left[\mathrm{GeV}^{-2}\right]$ & $20.6+6.5 i$ & $21.3+7.1 i$ & $21.1+6.8 i$ & $20.9+6.8 i$ & $22.1+7.2 i$ & $20.2+6.6 i$ \\
\hline$\left\langle r_{+1}^{2}\right\rangle\left[\mathrm{GeV}^{-2}\right]$ & $16.5+1.2 i$ & $16.9+1.3 i$ & $16.3+1.2 i$ & $17.1+1.3 i$ & $17.2+1.3 i$ & $16.3+1.2 i$ \\
\hline$G_{-1}(0)$ & $3.6-0.0 i$ & $3.4-0.0 i$ & $3.8-0.0 i$ & $3.2-0.0 i$ & $3.4-0.0 i$ & $3.6+0.0 i$ \\
\hline$\left\langle r_{-1}^{2}\right\rangle\left[\mathrm{GeV}^{-2}\right]$ & $16.3-1.1 i$ & $16.8-1.3 i$ & $16.0-1.1 i$ & $17.2-1.3 i$ & $17.0-1.2 i$ & $16.2-1.2 i$ \\
\hline$\Gamma_{\Sigma^{*} \rightarrow \Lambda \gamma}[\mathrm{MeV}]$ & 0.47 & 0.43 & 0.51 & 0.40 & 0.41 & 0.48 \\
\hline
\end{tabular}

cut located on the first Riemann sheet. The dashed lines correspond to $q^{2}$ values beyond the range of validity of $\chi \mathrm{PT}$. Moreover, our dispersion relations do not include any highenergy inelasticity, meaning that the TFF predictions can only be trusted at low $q^{2}$. Still, it can be illuminating to plot up to $q^{2}=-1 \mathrm{GeV}^{2}$. To accurately determine up to which $q^{2}$ the results are reliable, we would need a better knowledge about all the uncertainties involved, in order to be able to quantify where the errors start spreading beyond the desired precision. In particular, we would need to calculate the chiral corrections to the input pion-hyperon scattering amplitudes.

We plot the single differential decay width $d \Gamma / d q^{2}$ for the Dalitz decay $\Sigma^{*} \rightarrow \Lambda e^{+} e^{-}$, i.e., the angular integral of (22), in the Dalitz region $4 m_{e}^{2}<q^{2}<\left(m_{\Sigma^{*}}-m_{\Lambda}\right)^{2}$. In particular, in Fig. 4 we show a comparison with the corresponding QED case (24), for which the $q^{2}$ dependence of the TFFs is not resolved. The two curves show a slight offset in the central region, but essentially coincide over the whole range. This implies that high resolution is needed from the experimental

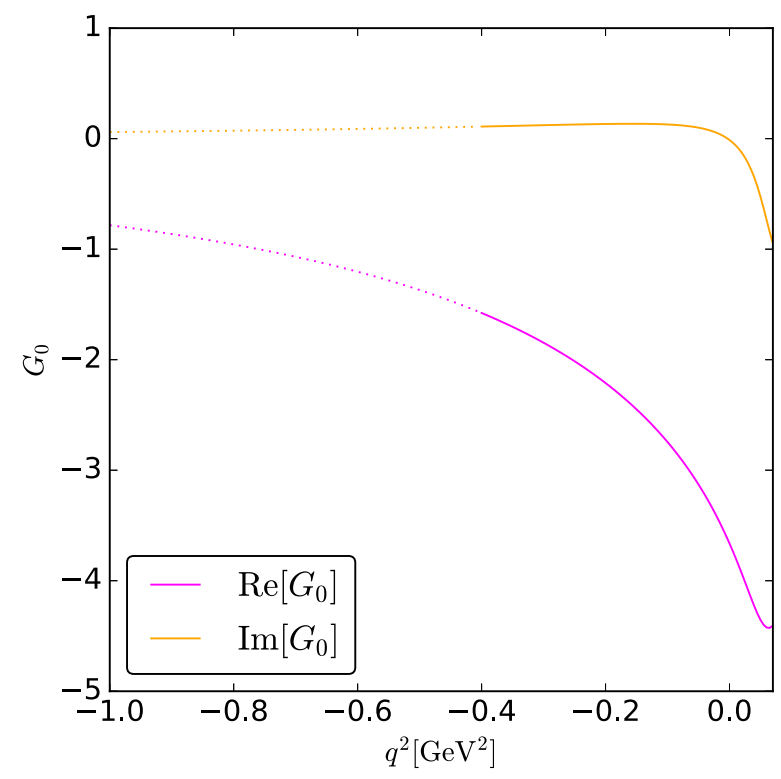

FIG. 1. Real and imaginary parts of $G_{0}\left(q^{2}\right)$. side in order to appreciate this discrepancy and gain new insight on the internal structure of hyperons.

In the Dalitz region, it is also meaningful to plot the three combinations of TFFs that appear in front of the trigonometric functions in the four-body decay expression (29), in order to compare their magnitude. Figure 5 shows that one of them, the linear combination of $\left|G_{+1}\right|^{2}$ and $\left|G_{-1}\right|^{2}$, is dominant, making it very challenging to extract information on the individual TFFs. Yet with sufficient statistics and angular resolution for the four-body decay $\Sigma^{*} \rightarrow \Lambda e^{+} e^{-} \rightarrow p \pi^{-} e^{+} e^{-}$one might get access to the smaller form factor combinations.

The situation might be compared to the history of the experimental determination of the pion-to-photon TFF and the corresponding radius from Dalitz decays $\pi^{0} \rightarrow \gamma e^{+} e^{-}$ as documented in the citations of Ref. [35]. Also, there one had to establish first the mere existence of the decay, then the approximate agreement with the QED case, and finally with much higher experimental efforts the existence of a nontrivial form factor. We are looking forward to this future endeavor for the hyperon sector.

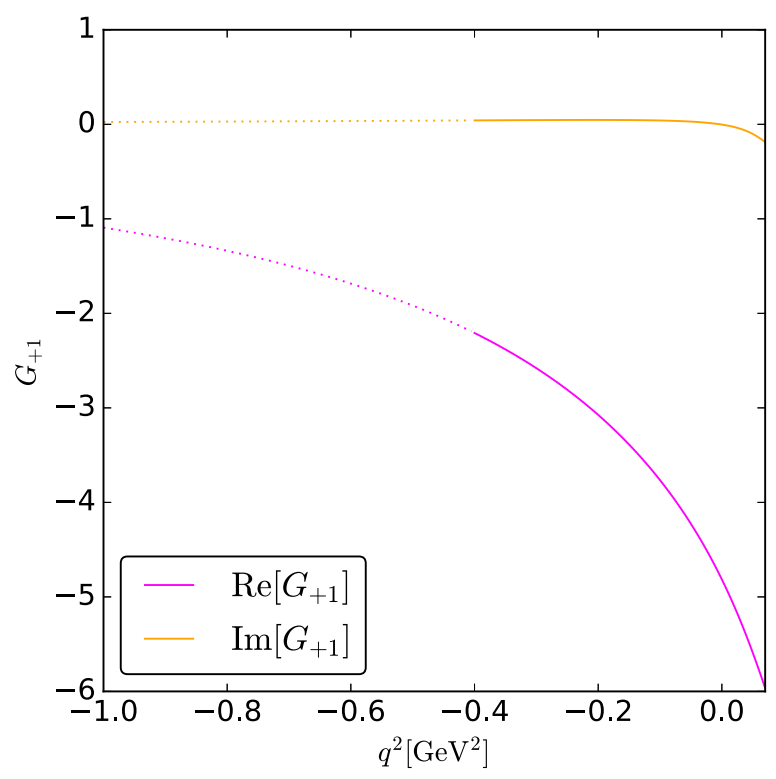

FIG. 2. Real and imaginary parts of $G_{+1}\left(q^{2}\right)$. 


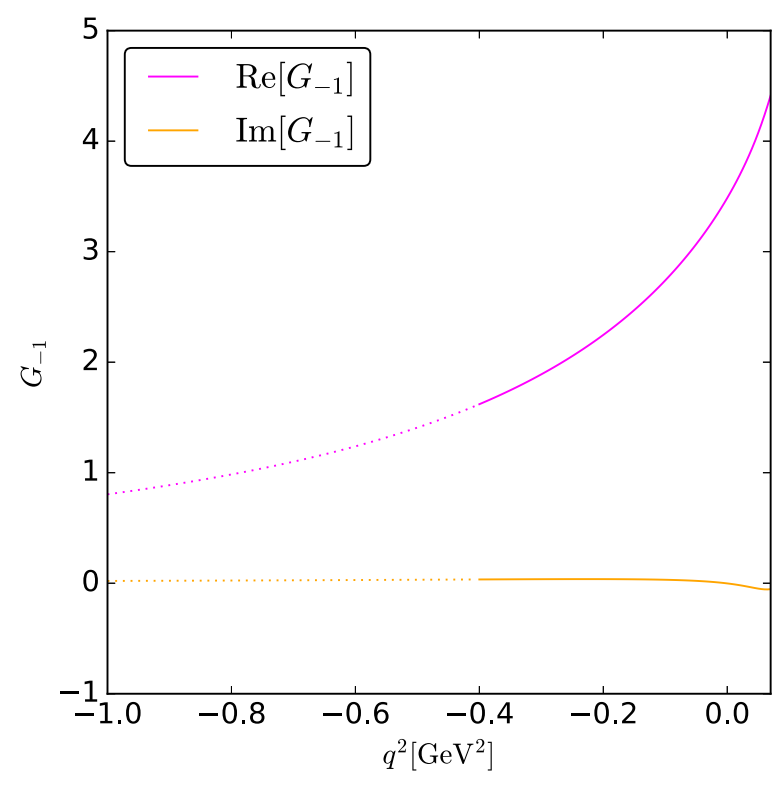

FIG. 3. Real and imaginary part of $G_{-1}\left(q^{2}\right)$.

\section{ACKNOWLEDGMENT}

S.L. thanks Martin Hoferichter and Bastian Kubis for very valuable discussions on anomalous thresholds.

\section{APPENDIX A: MESON VS BARYON DYNAMICS}

The purpose of this Appendix is to discuss the different physical aspects that are contained in a dispersive determination of the low-energy TFFs. As an integral part of the main text, it might distract the reader too much from the presentation of conceptual developments and results. Therefore, we dedicate this Appendix to this discussion.

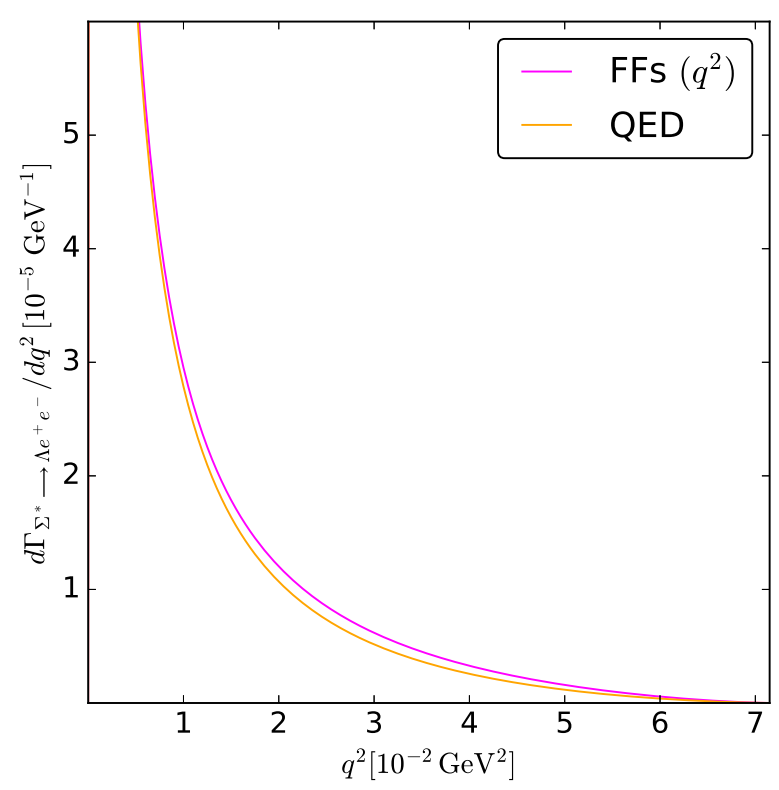

FIG. 4. Single-differential decay width for the $\Sigma^{*} \rightarrow \Lambda e^{+} e^{-}$ Dalitz decay. The curve labeled "FFs $\left(q^{2}\right)$ " is the angular integral of (22). The other curve is the QED analog, given by (24).

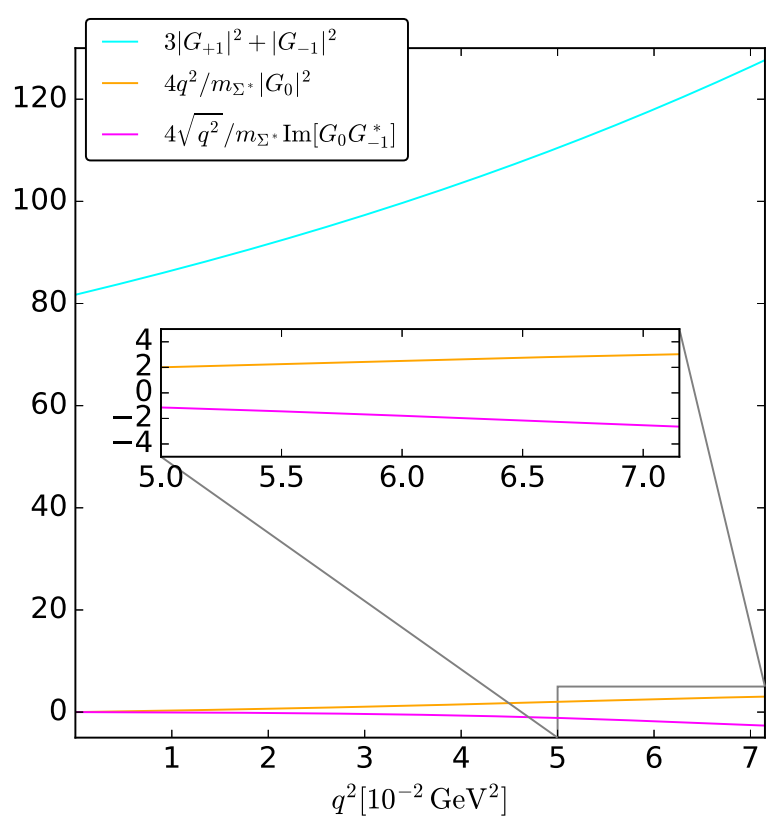

FIG. 5. A comparison of the three combinations of TFFs in front of the trigonometric functions in Eq. (29) for the $\Sigma^{*} \rightarrow p \pi^{-} e^{+} e^{-}$ decay.

To understand the physical content of our approach, it might be illuminating to study a form factor on the one-loop level. This is displayed in Fig. 6. Before discussing these diagrams, we stress that the dispersive approach contains more than these one-loop diagrams by including in Eq. (45) the complete rescattering of pions via their measured pion phase shift. The first diagram in Fig. 6 displays the exchange of a hyperon $Y$ in the crossed channels. The second diagram shows the formation of a vector meson $V$. The third diagram contains a contact interaction between the hyperons and the pions. A contact interaction is without structure. It contributes with a polynomial to the hyperon-pion scattering amplitude. Thus, the contact interaction provides a contribution to the polynomial $P$ in Eq. (45). For the following discussion we call this contribution $P_{c}$.

Next, we want to specify the relevant exchange hadrons $Y$ and $V$. If such a hadron is very heavy, its pole and cut structures caused by its propagator are not resolved. It contributes effectively like a contact interaction. Thus, what is not covered (at the one-loop level) by the third diagram of Fig. 6 are exchanges of light hadrons. Concerning the baryon exchange diagrams, we have included explicitly the relevant lightest baryon states from the octet and decuplet. We call the impact of these processes on the form factors the "aspect of baryon dynamics." Below, we will show a calculation that focuses only on this aspect. The second and third diagrams of Fig. 6 couple the external baryons directly to mesons. Therefore, we call the impact of these processes on the form factors the "aspect of meson dynamics." This part might be linked to the notion of vector meson dominance [32]. Below, we will also show a calculation that focuses only on this aspect.

Finally, let us look at the second diagram of Fig. 6 in more detail. The dynamics of the lightest vector meson, the $\rho$ 


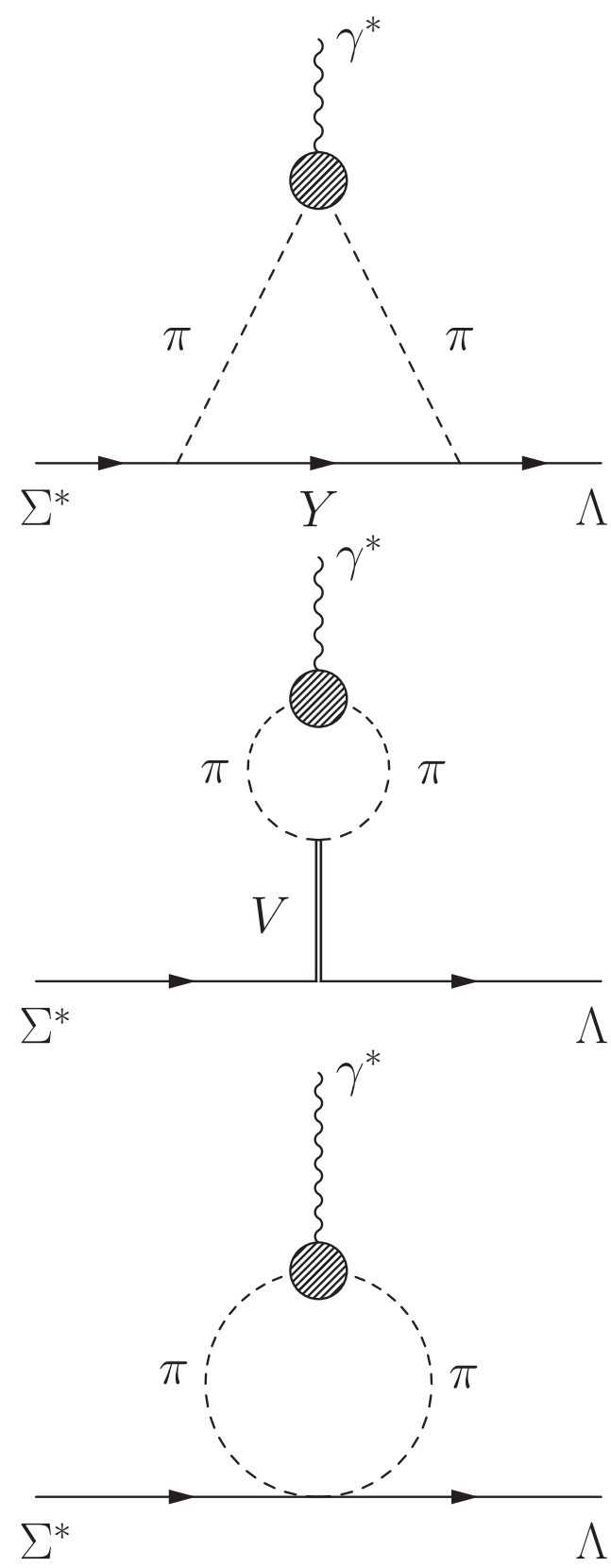

FIG. 6. One-loop diagrams contained in our approach. The shaded blob denotes the pion vector form factor. The first diagram leads to the amplitude $K$ in Eq. (45).

meson, is automatically contained in the measured pion phase shift because the $\rho$ meson couples essentially with $100 \%$ to a two-pion state. Diagrammatically, the second and third diagrams of Fig. 6 are covered by the diagram of Fig. 7.

What is not automatically covered is the initial coupling strength with which the vector meson $V$ couples the pions to the hyperons. Schematically,

$$
\begin{gathered}
i g_{B V} \bar{B} \gamma_{\mu} \gamma_{5} T_{\nu} V^{\mu \nu}+i G_{V}\left[u_{\mu}, u_{\nu}\right] V^{\mu \nu} \\
\rightarrow \frac{g_{B V} G_{V}}{M_{V}^{2}} \bar{B} \gamma_{\mu} \gamma_{5} T_{\nu}\left[u^{\mu}, u^{\nu}\right]
\end{gathered}
$$

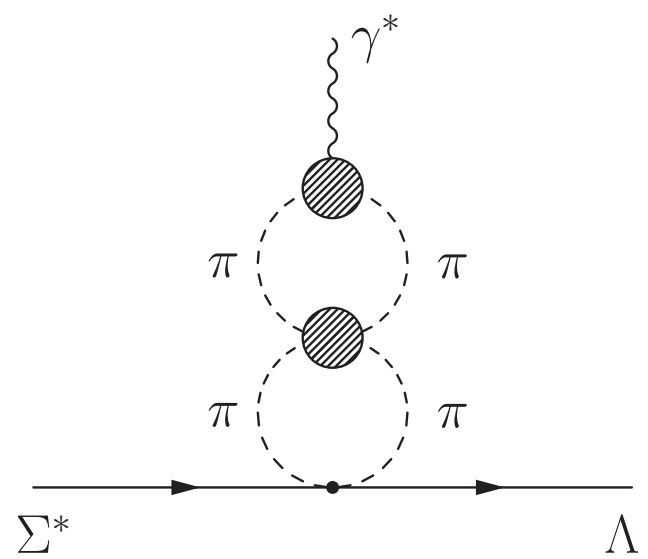

FIG. 7. Diagrammatic representation of all processes that do not contain the cross-channel exchange of light baryons. The shaded blob with four pion legs represents the $S$ matrix of pion scattering. The black dot contains the contact interaction of the third diagram of Fig. 6 and the strength mediated by the vector meson of the second diagram of Fig. 6. This black dot leads to the polynomial $P$ in Eq. (45).

which leads to

$$
P=P_{c}+P_{V} \quad \text { with } \quad P_{V} \sim \frac{g_{B V} G_{V}}{M_{V}^{2}} .
$$

In Appendix D and in Ref. [61], respectively, the flavor structure of (A1) is specified, which is, however, of no concern for our qualitative discussion. We will show below in more detail how the dynamics contained in the second diagram of Fig. 6 emerges from the dispersive framework by translating and simplifying this framework to the vector meson dominance language.

The result of the present discussion is that our dispersive framework contains all processes of Fig. 6 if the contact interaction strength $\sim P$ of Fig. 7 is determined by a fit to experiment. Without further theory input, this needs to be done separately for each form factor. If we need to estimate the size of $P$ on the theory side, we must include the influence of vector mesons as shown in Eq. (A2) and carried out in Appendix D. In this context, we note that a pion-hyperon contact term of a given order in $\chi$ PT leads to a contribution of the same order for the form factor. To be concrete, the TFFs $F_{i}$ of (2) start at second, third, and fourth chiral orders for $i=1,2,3$, respectively. Correspondingly, to fully account for the contribution of the $\rho$ meson to the TFF, $F_{i}$ requires a pion-hyperon contact interaction from the chiral Lagrangian of $(i+1)$ th order. With our present NLO input, we have a full coverage of $F_{1}$ only. In turn, $F_{1}$ constitutes the leading contribution to the TFFs $G_{ \pm 1}$ in Eqs. (8) and (10). In addition, our formalism contains the pertinent contributions to all TFFs from the baryon dynamics induced by the first diagram of Fig. 6.

Baryon form factors are influenced by meson dynamics and by baryon dynamics. Therefore, it might be illuminating to disentangle the meson and the baryon dynamics by switching off one of the two aspects. This will be discussed in the 


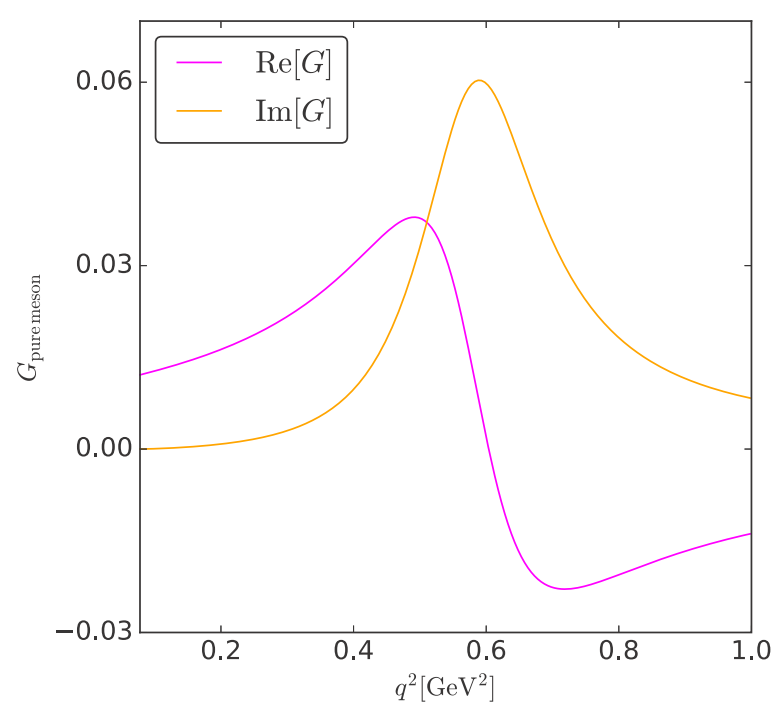

(a)

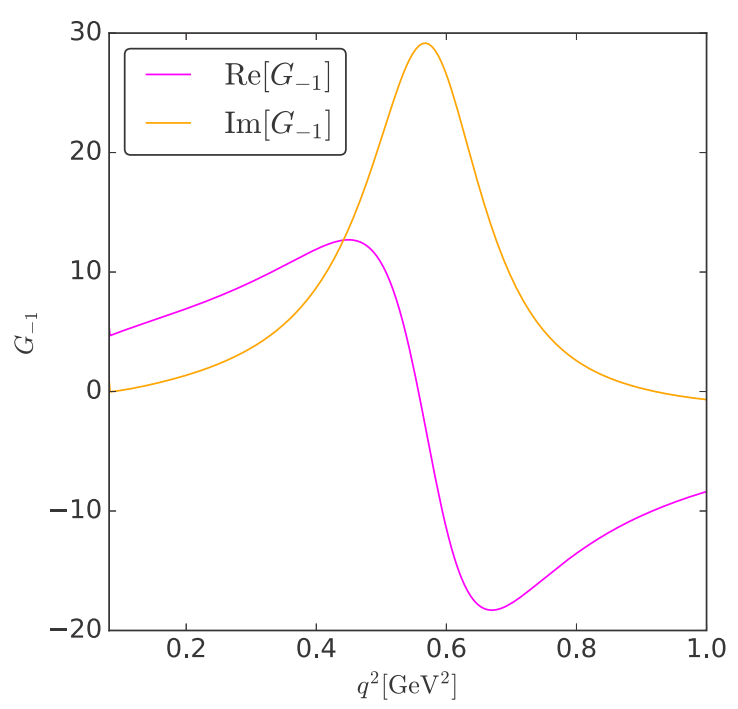

(b)

FIG. 8. Pure mesonic contribution to the TFFs (a) in arbitrary units, compared with the full $G_{-1}\left(q^{2}\right)$ described by meson and baryon dynamics together (b), in the unphysical timelike region $4 m_{\pi}^{2}<q^{2}<1 \mathrm{GeV}^{2}$.

following two subsections. We would like to stress that both cases miss part of the physics.

\section{Pure meson dynamics}

To focus on the impact of pion rescattering, we switch off the aspect of baryon dynamics, i.e., we put $K \rightarrow 0$ for the calculation of the hyperon-pion scattering amplitude $T$ in Eq. (45). Consequently, we put $f \rightarrow 0$ in Eqs. (53) and (60). For simplicity, we use the unsubtracted dispersion relation (64). Since we want to focus on the low-energy aspects, we also leave out the effective-pole term, i.e., $c_{m} \rightarrow 0$. Thus, we obtain finally

$$
G_{\text {pure meson }}\left(q^{2}\right)=\frac{P}{12 \pi} \int_{4 m_{\pi}^{2}}^{\infty} \frac{d s}{\pi} \frac{\Omega(s) p_{\mathrm{c} . \mathrm{m} .}^{3}(s) F_{\pi}^{V *}(s)}{s^{1 / 2}\left(s-q^{2}-i \epsilon\right)} .
$$

Since the subtraction constant $=$ contact interaction strength $P$ is just a number that does not influence the $s$ dependence of the integrand, we have not specified the TFF by any label. We show its generic form in Fig. 8(a), in the unphysical region between the two-pion threshold and $1 \mathrm{GeV}^{2}$. Obviously, the TFF displays the influence of the $\rho$ meson; i.e., the mesonic aspects are very well covered. An unphysical aspect emerges from the fact that the imaginary part of the TFF vanishes below the two-pion threshold. In reality, the TFF is complex everywhere, since the $\Sigma^{*}$ is unstable. This is, however, hardly visible in the full results for $G_{-1}$ in Fig. 8(b), since the imaginary part at $q^{2} \approx 4 m_{\pi}^{2}$ becomes extremely tiny. From the comparison of Figs. 8(a) and 8(b), one sees that if one adjusted the $\rho$ peak of the imaginary parts to the same size, then the peak of the real part of the full calculation will be somewhat smaller than the one of the pure-meson calculation. Moreover, at low energies the curvature in the real part of the full $G_{-1}$ is milder with respect to the pure-meson calculation.

A relation to strict vector meson dominance can be deduced from (A3). Suppose that the width of the vector meson is small. Essentially, this means that the pion phase shift is zero below the vector meson mass and $\pi$ above. This leads to $\Omega(s)=m_{\rho}^{2} /\left(m_{\rho}^{2}-s\right)$. With a slight refinement, $m_{\rho}^{2}-s \rightarrow$ $m_{\rho}^{2}-s-i m_{\rho} \Gamma_{\rho}$, one obtains $\Omega(s) F_{\pi}^{V *}(s) \sim \delta\left(s-m_{\rho}^{2}\right) / \Gamma_{\rho}$ and therefore $G_{\text {pure meson }}\left(q^{2}\right) \sim\left(P / \Gamma_{\rho}\right) /\left(m_{\rho}^{2}-q^{2}\right)$. The appearance of the ratio $P / \Gamma_{\rho}$ has a natural interpretation: In a vector meson dominance picture, the contact term $\sim P$ for the hyperon-pion scattering amplitude emerges from integrating out the vector meson; see (A1) and (A2). This leads to $P \sim$ $g_{B \rho} G_{V}$. On the other hand, in strict vector meson dominance, the coupling of the vector meson to the pions must be adjusted such that the correct electric charge of the pion emerges that is independent of strong-interaction coupling constants, i.e., $G_{V} \sim 1 / F_{V}$, where $F_{V}$ denotes the coupling strength with which the photon couples to the vector meson. Thus, one finds $P / \Gamma_{\rho} \sim P / G_{V}^{2} \sim g_{B \rho} / G_{V} \sim g_{B \rho} F_{V}$. This is exactly what one expects as the coefficient of a form factor obtained in the vector meson dominance framework; i.e., in full analogy to (A1) one finds

$$
i g_{B V} \bar{B} \gamma_{\mu} \gamma_{5} T_{v} V^{\mu \nu}+F_{V} f_{+}^{\mu \nu} V_{\mu v} \rightarrow i \frac{g_{B V} F_{V}}{M_{V}^{2}} \bar{B} \gamma_{\mu} \gamma_{5} T_{v} f_{+}^{\mu \nu}
$$

Thus, the dispersive framework reproduces and refines the vector meson dominance aspects. The only input one needs is the initial strength (contact interaction) with which the pions couple to the baryons. In the vector meson dominance setup, this is obtained by integrating out the vector meson. One can also rephrase it in the following way: If one integrates out the vector meson for the interaction terms between the vector meson and the baryons and between the vector meson and the photon, one obtains a photon-baryon coupling right away. The dispersive framework produces the same with the pions as intermediate agents. Of course, the dispersive framework based on data for the pion phase shift and the pion vector 


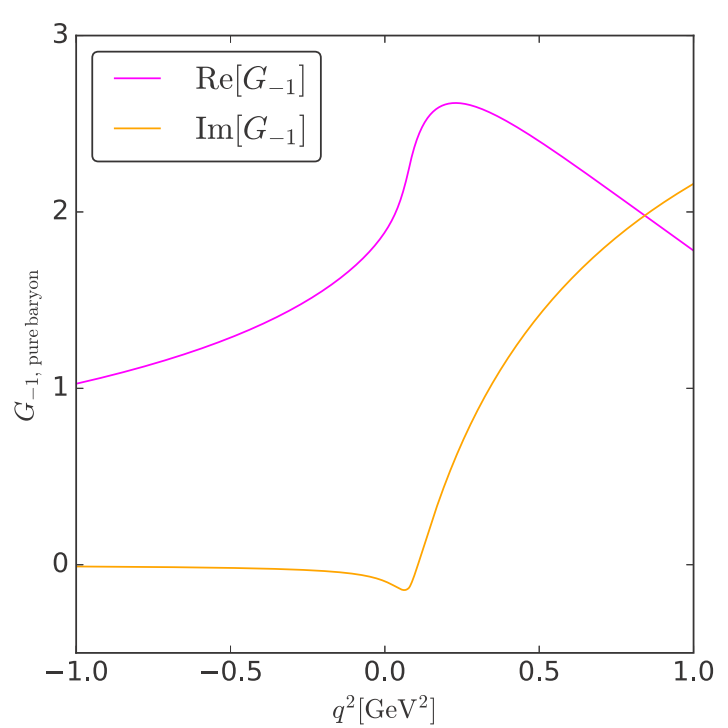

(a)

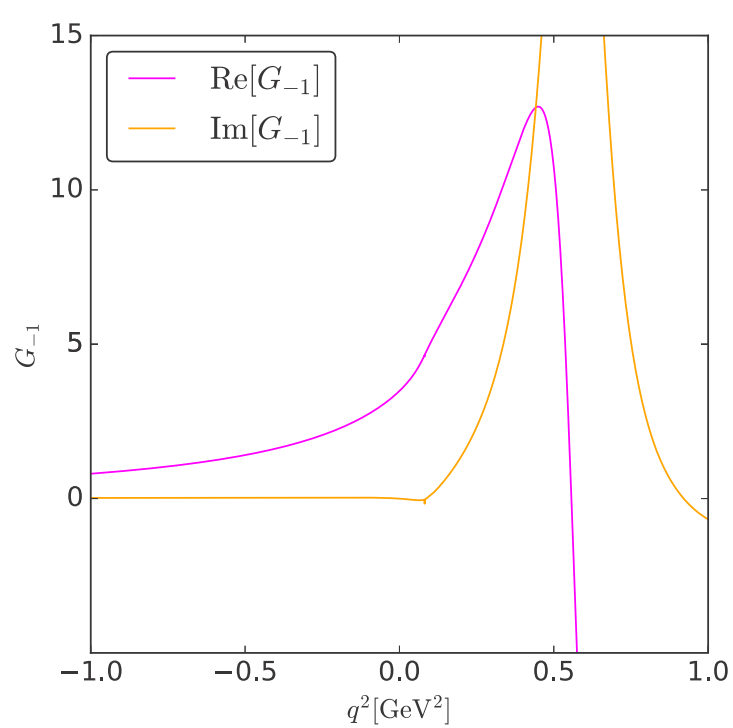

(b)

FIG. 9. Pure baryonic contribution to $G_{-1}\left(q^{2}\right)$ (a) as compared to the full result for $G_{-1}\left(q^{2}\right)$ described by meson and baryon dynamics together (b), in the range $-1<q^{2}\left[\mathrm{GeV}^{2}\right]<1$.

form factor is more accurate than the schematic and modeldependent vector meson dominance scenario but it covers qualitatively the same physics. In addition, the dispersive framework presented in this paper contains also the aspects of baryon dynamics that is completely missing in the vector meson dominance approach.

\section{Pure baryon dynamics}

To focus on the impact of the processes where baryons are exchanged in the cross channel, we switch off the contact interaction and the pion rescattering. For simplicity, we use an unsubtracted dispersion relation. Thus, we put $F_{\pi}^{V} \rightarrow 1$ in Eqs. (64) and (60). Since we want to focus on the lowenergy aspects, we also leave out the effective-pole term, i.e., $c_{m} \rightarrow 0$. For the calculation of the scattering amplitude $T$, we put $P \rightarrow 0$ and $\delta \rightarrow 0$ in Eq. (45). This also implies that the anomalous piece vanishes. In simple terms, $T_{m} \rightarrow K_{m}$ in Eq. (64). This leads to

$$
\begin{aligned}
G_{m}^{\text {pure baryon }}\left(q^{2}\right)= & \frac{1}{12 \pi} \int_{4 m_{\pi}^{2}}^{\Lambda^{2}} \frac{d s}{\pi} \frac{K_{m}(s) p_{\mathrm{c} . \mathrm{m} .}^{3}(s)}{s^{1 / 2}\left(s-q^{2}-i \epsilon\right)} \\
& +\frac{1}{12 \pi} \int_{0}^{1} d x \frac{d s^{\prime}(x)}{d x} \frac{1}{s^{\prime}(x)-q^{2}} \\
& \times \frac{f_{m}\left(s^{\prime}(x)\right) s^{\prime}(x)}{-4\left[-\lambda\left(s^{\prime}(x), m_{\Sigma^{*}}^{2}, m_{\Lambda}^{2}\right)\right]^{3 / 2}} .
\end{aligned}
$$

In Fig. 9(a), this contribution is plotted for the TFF $G_{-1}\left(q^{2}\right)$, in the range $-1<q^{2}\left[\mathrm{GeV}^{2}\right]<1$. As expected, the form factor has an imaginary part for all values of $q^{2}$, even if very tiny for $q^{2}<0$. The baryon exchange diagrams contain the physical aspect that the $\Sigma^{*}$ is unstable. What is missing, of course, is the influence of the $\rho$ meson, i.e., the mesonic aspects. For comparison, we show in Fig. 9(b) also the complete result for $G_{-1}\left(q^{2}\right)$, taking into account the contributions of both meson and baryon dynamics, again across the spaceand timelike regions. Note that the same quantity has been previously plotted [Fig. 8(b)] but in a different range. This time, we include the negative $q^{2}$ physical region and focus on the region around $q^{2}=0$. There, we notice in Fig. 9(a) a steep rise in the real part, which is mitigated in Fig. 9(b) by the $\rho$-meson tail. In summary, even if the $\rho$-meson dictates in general the shape and size of the form factor, the low-energy behavior is significantly influenced by the baryon dynamics.

\section{APPENDIX B: PROJECTOR FORMALISM FOR HELICITY AMPLITUDES}

Spin- $3 / 2$ objects can be obtained from the coupling of spin- $1 / 2$ and spin- 1 states. Thus, we construct a spin-3/2 vector-spinor [62] by

$$
u^{\mu}(p, \sigma)=\sum_{\rho, \lambda}\left(\frac{3}{2}, \sigma \mid 1, \rho ; \frac{1}{2}, \lambda\right) u(p, \lambda) \varepsilon^{\mu}(p, \rho)
$$

with a spin-1/2 spinor $u$, a spin-1 polarization vector $\varepsilon^{\mu}$ and a Clebsch-Gordan coefficient $\left(J, M \mid j_{1}, m_{1} ; j_{2}, m_{2}\right)$. Here, in slight contrast to the rest of this work, the spin projections on a given quantization axis (and not the helicities) are denoted by $\sigma, \lambda$, and $\rho$, respectively. Yet if one chooses the quantization axis along the flight direction (as we will do in a moment) then helicity and spin projection coincide.

It is useful to provide (B1) in an explicit form:

$$
\begin{aligned}
u^{\mu}(p, \pm 3 / 2)= & u(p, \pm 1 / 2) \varepsilon^{\mu}(p, \pm 1), \\
u^{\mu}(p, \pm 1 / 2)= & \frac{1}{\sqrt{3}} u(p, \mp 1 / 2) \varepsilon^{\mu}(p, \pm 1) \\
& +\frac{\sqrt{2}}{\sqrt{3}} u(p, \pm 1 / 2) \varepsilon^{\mu}(p, 0) .
\end{aligned}
$$


For the spin-1/2 spinors, we use the conventions of Ref. [38]. For the spin-1 polarization vectors for massive states, we provide here only their explicit form for the case where the $z$ direction constitutes both the spin quantization axis and the direction of motion of the particle $[63,64]$ :

$$
\begin{aligned}
\varepsilon^{\mu}\left(p_{z}, \pm 1\right) & =\frac{\mp 1}{\sqrt{2}}(0,1, \pm i, 0), \\
\varepsilon^{\mu}\left(p_{z}, 0\right) & =\left(p_{z} / m, 0,0, E / m\right),
\end{aligned}
$$

where $m$ denotes the mass of the particle and $E$ its energy. Note that the coefficient $\mp 1$ does not show up in the definitions of Ref. [38].

Irrespective of flight direction and spin quantization axis, the spin- $3 / 2$ vector-spinors satisfy

$$
\sum_{\sigma} u_{\mu}(p, \sigma) \bar{u}_{v}(p, \sigma)=-(\not p+m) P_{\mu \nu}^{3 / 2}(p),
$$

where $p^{0}=\sqrt{m^{2}+\vec{p}^{2}}$ denotes the energy of the particle described by the vector-spinor and $m$ its mass. The projector on spin $3 / 2$ is defined by

$$
P_{\mu \nu}^{3 / 2}(p):=g_{\mu \nu}-\frac{1}{3} \gamma_{\mu} \gamma_{\nu}-\frac{1}{3 p^{2}}\left(\not p \gamma_{\mu} p_{\nu}+p_{\mu} \gamma_{\nu} \not p\right) .
$$

Note that for (B4) the scalar product $p^{2}$ appearing in Eq. (B5) can be replaced by $m^{2}$.

For our Lagrangian (67), a spin-3/2 (vector-spinor) field $\psi_{\mu}(x)$ has the following propagator $[65,66]$

$$
\left\langle 0\left|\mathrm{~T} \psi_{\mu}(x) \bar{\psi}_{\nu}(y)\right| 0\right\rangle=\int \frac{d^{4} p}{(2 \pi)^{4}} i S_{\mu \nu}(p) e^{-i p(x-y)}
$$

with

$$
\begin{aligned}
S_{\mu \nu}(p):= & -\frac{\not p+m}{p^{2}-m^{2}+i \epsilon} P_{\mu \nu}^{3 / 2}(p)+\frac{2}{3 m^{2}}(\not p+m) \frac{p_{\mu} p_{v}}{p^{2}} \\
& -\frac{1}{3 m} \frac{p_{\mu} p^{\alpha} \gamma_{\alpha \nu}+\gamma_{\mu \alpha} p^{\alpha} p_{v}}{p^{2}} .
\end{aligned}
$$

Note that for the propagator of (B6) and (B7), the scalar product $p^{2}$ appearing in Eq. (B5) cannot be replaced by $m^{2}$. The propagator (B7) describes propagating modes of spin $3 / 2$ and frozen modes of spin $1 / 2$ [65].

The scattering amplitudes for the reaction $\Sigma^{*} \bar{\Lambda} \rightarrow \pi^{+} \pi^{-}$ have the following structure:

$$
\bar{v}_{\Lambda}\left(p_{\Lambda}, \lambda\right) M_{\mu}\left(p_{\Sigma^{*}}, p_{\Lambda}, k\right) u_{\Sigma^{*}}^{\mu}\left(p_{\Sigma^{*}}, \sigma\right)
$$

with $k:=p_{\pi^{+}}-p_{\pi^{-}}$. Feynman rules can provide a quite lengthy expression for the spinor $4 \times 4$ matrix $M^{\mu}$. Therefore, we aim at a projector formalism where (B8) is related to scalar quantities $a_{i}$ and a predefined set of spinor objects such that only the scalar quantities depend on the explicit form of $M^{\mu}$, i.e.,

$$
\bar{v}_{\Lambda} M_{\mu} u_{\Sigma^{*}}^{\mu}=\sum_{i} a_{i} \bar{v}_{\Lambda} M_{i}^{\mu} g_{\mu \nu} u_{\Sigma^{*}}^{v}
$$

The tasks are to construct a complete set of linearly independent structures $M_{i}^{\mu}$ and to find a convenient way to determine $a_{i}$ from an arbitrary $M^{\mu}$. Such an endeavor is similar in spirit to Ref. [64].
Because of parity symmetry, we can focus on the case where the $\bar{\Lambda}$ has positive helicity, $\lambda=+1 / 2$. Then we need four predefined spinor objects corresponding to the possible values for the helicity of the $\Sigma^{+}$baryon, $\sigma=$ $+3 / 2,+1 / 2,-1 / 2,-3 / 2$. It is convenient to introduce the following four-vectors:

$$
\begin{aligned}
q & :=p_{\Sigma^{*}}+p_{\Lambda}, \\
\bar{k} & :=p_{\Sigma^{*}}-p_{\Lambda}, \\
r & :=\bar{k}-\frac{\bar{k} \cdot q}{q^{2}} q, \\
k_{\perp} & :=k-\frac{k \cdot r}{r^{2}} r .
\end{aligned}
$$

In the center-of-mass frame with the three-momentum of the $\Sigma^{*}$ pointing in the $z$ direction and the reaction taking place in the $x-z$ plane, one finds that $q$ has only a zeroth component, $r$ has only a third $(z)$ component, and $k_{\perp}$ has only a first $(x)$ component.

We are looking now for four independent structures of type $M^{\mu}$ in Eq. (B8). In general, $M^{\mu}$ contains products of $\gamma$ matrices and exactly one $\gamma_{5} .{ }^{7}$ All $\gamma$ matrices that are contracted with $p_{\Lambda}, p_{\Sigma^{*}}$ or the spin-3/2 spinor $u_{\Sigma^{*}}$ can be moved toward $\bar{v}_{\Lambda}$ or $u_{\Sigma^{*}}$ and eliminated by equations of motion. This results in structures with fewer $\gamma$ matrices. If two $\gamma$ matrices are contracted with each other or with the very same four-momentum, then one can also simplify the expression.

This whole procedure leaves us with four independent structures of $M^{\mu}$ type:

$$
\gamma_{5} k_{\perp}^{\mu}, \quad \gamma_{5} p_{\Lambda}^{\mu}, \quad k_{\perp} \gamma_{5} p_{\Lambda}^{\mu}, \quad k_{\perp} \gamma_{5} k_{\perp}^{\mu} .
$$

It is simpler, however, to use the following linear combinations:

$$
\begin{aligned}
& M_{1}^{\mu}:=\left[q^{2}-\left(m_{\Sigma^{*}}+m_{\Lambda}\right)^{2}\right] \gamma_{5} k_{\perp}^{\mu}-m_{\Sigma^{*}} k_{\perp} \gamma_{5} p_{\Lambda}^{\mu}, \\
& M_{2}^{\mu}:=\gamma_{5} p_{\Lambda}^{\mu}, \\
& M_{3}^{\mu}:=k_{\perp} \gamma_{5} p_{\Lambda}^{\mu}, \\
& M_{4}^{\mu}:=\left[q^{2}-\left(m_{\Sigma^{*}}-m_{\Lambda}\right)^{2}\right] k_{\perp} \gamma_{5} k_{\perp}^{\mu}-m_{\Sigma^{*}} k_{\perp}^{2} \gamma_{5} p_{\Lambda}^{\mu} .
\end{aligned}
$$

They are constructed such that in the center-of-mass frame they satisfy

$$
\bar{v}_{\Lambda}\left(p_{\Lambda},+1 / 2\right) M_{i}^{\mu} g_{\mu \nu} u_{\Sigma^{*}}^{v}\left(p_{\Sigma^{*}}, \sigma\right) \sim \delta_{i i_{\Sigma^{*}}}
$$

with $i_{\Sigma^{*}}:=5 / 2-\sigma$. In other words, each $M_{i}^{\mu}$ contributes only to one helicity amplitude. Thus, the sum in Eq. (B9) reduces to only one term.

The remaining task is to find the scalar quantity $a_{i}$ for a given $M^{\mu}$. What makes the task nontrivial is the fact that different $M^{\mu}$ lead to the same $a_{i}$ because of the equations of motion for $\bar{v}_{\Lambda}$ and $u_{\Sigma^{*}}^{v}$. Therefore, we construct on- and off-shell projectors to decompose a completely general $M^{\mu}$. Since $M^{\mu}$ is a $4 \times 4$ spinor matrix with $\mu$ ranging from 0 to

\footnotetext{
${ }^{7}$ Note that alternatively to a $\gamma_{5}$ one might involve a Levi-Civita symbol. However, this can be related to one $\gamma_{5}$ and a product of $\gamma$ matrices.
} 
3 we need in general a basis of 64 Lorentz-spinor structures. Because of parity symmetry, we can restrict ourselves to 32 structures. For the first four terms, we use

$$
T_{\mu}^{i}:=P_{\mathrm{on}}^{\Lambda} M_{i}^{v} P_{\mathrm{on}}^{\Sigma^{*}} P_{v \mu}^{3 / 2},
$$

introducing the projectors [65]

$$
\begin{aligned}
P_{\mathrm{on}}^{\Lambda} & :=\frac{1}{2 m_{\Lambda}}\left(m_{\Lambda}-\not p_{\Lambda}\right), \\
P_{\mathrm{off}}^{\Lambda} & :=\frac{1}{2 m_{\Lambda}}\left(m_{\Lambda}+\not p_{\Lambda}\right), \\
P_{\mathrm{on}}^{\Sigma^{*}} & :=\frac{1}{2 m_{\Sigma^{*}}}\left(m_{\Sigma^{*}}+\not p_{\Sigma^{*}}\right), \\
P_{\mathrm{off}}^{\Sigma^{*}} & :=\frac{1}{2 m_{\Sigma^{*}}}\left(m_{\Sigma^{*}}-\not p_{\Sigma^{*}}\right), \\
P_{\mu \nu}^{1 / 2} & :=\frac{1}{3} \gamma_{\mu} \gamma_{\nu}+\frac{1}{3 p_{\Sigma^{*}}^{2}}\left(\not p_{\Sigma^{*}} \gamma_{\mu} g_{\nu \alpha}+g_{\mu \alpha} \gamma_{\nu} \not p_{\Sigma^{*}}\right) p_{\Sigma^{*}}^{\alpha}, \\
P_{\mu \nu}^{3 / 2} & :=g_{\mu \nu}-P_{\mu \nu}^{1 / 2} .
\end{aligned}
$$

The other 28 structures are obtained from (B14) by exchanging $P_{\text {on }}^{\Lambda}$ by $P_{\text {off }}^{\Lambda}$ and/or $P_{\text {on }}^{\Sigma^{*}}$ by $P_{\text {off }}^{\Sigma^{*}}$ and/or $P_{v \mu}^{3 / 2}$ by $P_{v \mu}^{1 / 2}$. We do not specify how we enumerate these structures from $i=5$ to $i=32$ because we will not need them in the end. We also introduce the Dirac adjoint structures

$$
\bar{T}_{\mu}^{i}:=\gamma_{0}\left(T_{\mu}^{i}\right)^{\dagger} \gamma_{0}, \quad \text { for } i=1, \ldots, 32 .
$$

Provided that the $T_{\mu}^{i}$ form 32 linearly independent structures, we can decompose any $M_{\mu}$ as

$$
M_{\mu}=\sum_{i=1}^{32} a_{i} T_{\mu}^{i}
$$

with

$$
a_{i}=\sum_{j=1}^{32}\left(C^{-1}\right)_{i j} \operatorname{Tr}\left(\bar{T}_{\mu}^{j} M^{\mu}\right)
$$

and the $32 \times 32$ matrix $C$ with elements

$$
C_{i j}:=\operatorname{Tr}\left(\bar{T}_{\mu}^{i} T_{\nu}^{j}\right) g^{\mu \nu} .
$$

Here $\operatorname{Tr}$ denotes the spinor trace. We have checked explicitly that $\operatorname{det} C \neq 0$ which shows that the 32 structures $T_{\mu}^{i}$ are linearly independent, i.e., form a basis to construct the most general $M_{\mu}$.

Inserting (B17) in Eq. (B8) and using the equations of motion for the spinors shows

$$
\bar{v}_{\Lambda} M_{\mu} u_{\Sigma^{*}}^{\mu}=\sum_{i=1}^{4} a_{i} \bar{v}_{\Lambda} M_{i}^{\mu} g_{\mu \nu} u_{\Sigma^{*}}^{\nu}
$$

Thus, we only need to determine the four scalar quantities $a_{i}$ with $i=1,2,3,4$ from (B18). Since the projectors in Eq. (B15) are pairwise orthogonal, one finds

$$
\operatorname{Tr}\left(\bar{T}_{\mu}^{i} T_{v}^{j}\right) g^{\mu \nu}=0 \quad \text { for } i>4, j=1,2,3,4 .
$$

In addition, we have checked by an explicit calculation

$$
C_{i j} \sim \delta_{i j} \quad \text { for } \quad i, j=1,2,3,4,
$$

a result that one could have anticipated already from (B13). Finally, (B18) simplifies to

$$
a_{i}=\frac{\operatorname{Tr}\left(\bar{T}_{\mu}^{i} M^{\mu}\right)}{C_{i}}
$$

with

$$
C_{i}:=\operatorname{Tr}\left(\bar{T}_{\mu}^{i} T_{\nu}^{i}\right) g^{\mu \nu}
$$

Explicit expressions are given by

$$
\begin{aligned}
C_{1} & :=\frac{k_{\perp}^{2}}{4 m_{\Sigma^{*}} m_{\Lambda}}\left[\left(m_{\Sigma^{*}}+m_{\Lambda}\right)^{2}-q^{2}\right] \lambda\left(q^{2}, m_{\Sigma^{*}}^{2}, m_{\Lambda}^{2}\right), \\
C_{2} & :=\frac{-1}{12 m_{\Sigma^{*}}^{3} m_{\Lambda}}\left[\left(m_{\Sigma^{*}}-m_{\Lambda}\right)^{2}-q^{2}\right] \lambda\left(q^{2}, m_{\Sigma^{*}}^{2}, m_{\Lambda}^{2}\right), \\
C_{3} & :=\frac{k_{\perp}^{2}}{12 m_{\Sigma^{*}}^{3} m_{\Lambda}}\left[\left(m_{\Sigma^{*}}+m_{\Lambda}\right)^{2}-q^{2}\right] \lambda\left(q^{2}, m_{\Sigma^{*}}^{2}, m_{\Lambda}^{2}\right), \\
C_{4} & :=\frac{-\left(k_{\perp}^{2}\right)^{2}}{4 m_{\Sigma^{*}} m_{\Lambda}}\left[\left(m_{\Sigma^{*}}-m_{\Lambda}\right)^{2}-q^{2}\right] \lambda\left(q^{2}, m_{\Sigma^{*}}^{2}, m_{\Lambda}^{2}\right)
\end{aligned}
$$

with the Källén function defined in Eq. (12). In the center-ofmass frame, one finds

$$
k_{\perp}^{2}=-4 p_{\text {c.m. }}^{2} \sin ^{2} \theta
$$

with the center-of-mass momentum of the pions $p_{\text {c.m. }}:=$ $\sqrt{q^{2}-4 m_{\pi}^{2}} / 2$ and $\theta$ denoting the angle between the threemomenta of $\Sigma^{*}$ and $\pi^{+}$.

To summarize, for a given amplitude structure $M_{\mu}$ and a given helicity $\sigma$, we find in the center-of-mass frame

$$
\begin{aligned}
& \bar{v}_{\Lambda}\left(p_{\Lambda},+1 / 2\right) M_{\mu} u_{\Sigma^{*}}^{\mu}\left(p_{\Sigma^{*}}, \sigma\right) \\
& =\frac{\operatorname{Tr}\left(\bar{T}_{\alpha}^{i} M^{\alpha}\right)}{C_{i}} \bar{v}_{\Lambda}\left(p_{\Lambda},+1 / 2\right) M_{i}^{\mu} g_{\mu \nu} u_{\Sigma^{*}}^{\nu}\left(p_{\Sigma^{*}}, \sigma\right)
\end{aligned}
$$

with $i=5 / 2-\sigma$. Note in particular that in Eq. (B27) there is no implicit summation over $i$; it is fixed by the choice of $\sigma$, the helicity of the $\Sigma^{*}$.

In the main text, we have introduced reduced amplitudes (44) for the dispersive representation of the TFFs. To make contact with these reduced amplitudes, we present here the ratios

$$
\begin{gathered}
\frac{\bar{v}_{\Lambda}\left(-p_{z},+1 / 2\right) M_{1}^{\mu} g_{\mu \nu} u_{\Sigma^{*}}^{v}\left(p_{z},+3 / 2\right)}{\bar{v}_{\Lambda}\left(-p_{z},+1 / 2\right) \gamma_{5} u_{\Sigma^{*}}^{1}\left(p_{z},+3 / 2\right) p_{\mathrm{c} . \mathrm{m} .}} \\
=-2 \sin \theta\left[q^{2}-\left(m_{\Sigma^{*}}+m_{\Lambda}\right)^{2}\right], \\
\frac{\bar{v}_{\Lambda}\left(-p_{z},+1 / 2\right) M_{2}^{\mu} g_{\mu \nu} u_{\Sigma^{*}}^{v}\left(p_{z},+1 / 2\right)}{\bar{v}_{\Lambda}\left(-p_{z},+1 / 2\right) \gamma_{5} u_{\Sigma^{*}}^{3}\left(p_{z},+1 / 2\right) p_{\mathrm{c} . \mathrm{m} .}} \\
=\frac{2 q^{2}}{m_{\Sigma^{*}}^{2}-m_{\Lambda}^{2}+q^{2}} \frac{p_{z}}{p_{\mathrm{c} . \mathrm{m} .}}, \\
\frac{\bar{v}_{\Lambda}\left(-p_{z},+1 / 2\right) M_{3}^{\mu} g_{\mu \nu} u_{\Sigma^{*}}^{v}\left(p_{z},-1 / 2\right)}{\bar{v}_{\Lambda}\left(-p_{z},+1 / 2\right) \gamma_{5} u_{\Sigma^{*}}^{1}\left(p_{z},-1 / 2\right) p_{\mathrm{c} . \mathrm{m} .}} \\
=-2 \sin \theta \frac{q^{2}-\left(m_{\Sigma^{*}}+m_{\Lambda}\right)^{2}}{m_{\Sigma^{*}}}
\end{gathered}
$$


with $p_{z}:=\lambda^{1 / 2}\left(q^{2}, m_{\Sigma^{*}}^{2}, m_{\Lambda}^{2}\right) /\left(2 \sqrt{q^{2}}\right)$ denoting the center-ofmass momentum of $\Sigma^{*}$ and $\bar{\Lambda}$. Note that for the $M_{2}$ case (nonflip amplitude) there will always be a factor $p_{z} p_{\text {c.m. }}$ from the partial-wave projection of $\operatorname{Tr}\left(\bar{T}_{\alpha}^{2} M^{\alpha}\right)$. Together with the last ratio on the right-hand side of the corresponding equation in Eq. (B28), this leads to an expression for the reduced amplitude without any square roots.

In practice, the whole task of dealing with a Feynman scattering amplitude for given helicities is reduced to the calculation of one spinor trace $\operatorname{Tr}\left(\bar{T}_{\alpha}^{i} M^{\alpha}\right)$.

\section{APPENDIX C: DISPERSIVE REPRESENTATIONS, CUTS AND ANOMALOUS THRESHOLDS}

This Appendix has two purposes. First, we provide a detailed discussion of the analytic structure of a scalar triangle diagram. This resembles the first diagram shown in Fig. 6, except that one deals with $p$ waves there and with $s$ waves in the scalar case. But the appearance of anomalous thresholds has the very same pattern. Therefore, we use the scalar triangle as a test case to check that we include all bits and pieces in the correct way for our TFF calculations. The second purpose is the derivation of (53), (60), and (63).

Consider the result of a triangle loop diagram [44,67-70],

$C(s)$

$$
=\frac{1}{i \pi^{2}} \int d^{4} l \frac{1}{\left(l^{2}-m_{\mathrm{exch}}^{2}\right)\left[\left(l+p_{1}\right)^{2}-m_{\pi}^{2}\right]\left[\left(l-p_{2}\right)^{2}-m_{\pi}^{2}\right]},
$$

which can be calculated directly when rewritten as

$$
\begin{aligned}
C(s)= & \int_{0}^{1} d x_{1} d x_{2} d x_{3} \delta\left(1-x_{1}-x_{2}-x_{3}\right)\left[x_{1} x_{3} m_{1}^{2}+x_{2} x_{3} m_{2}^{2}\right. \\
& \left.+x_{1} x_{2} s-x_{1} m_{\pi}^{2}-x_{2} m_{\pi}^{2}-x_{3} m_{\mathrm{exch}}^{2}\right]^{-1}
\end{aligned}
$$

with $s:=\left(p_{1}+p_{2}\right)^{2}, m_{1}^{2}:=p_{1}^{2}$, and $m_{2}^{2}:=p_{2}^{2}$. We consider first the case that $m_{\text {exch }}^{2}$ is large enough and $m_{1}^{2}$ and $m_{2}^{2}$ are small enough. A quantitative specification will follow later. In this case, the imaginary part of $C$ (for real values of $s$ ) is just given by cutting [71] the two pion lines of the Feynman diagram. The result is

$$
\operatorname{Im} C(s)=-\pi \frac{\sigma(s)}{\kappa(s)} \log \frac{Y(s)+\kappa(s)}{Y(s)-\kappa(s)} \Theta\left(s-4 m_{\pi}^{2}\right),
$$

where

$$
\begin{aligned}
& Y(s):=s+2 m_{\mathrm{exch}}^{2}-m_{1}^{2}-m_{2}^{2}-2 m_{\pi}^{2}, \\
& \kappa(s):=\lambda^{1 / 2}\left(s, m_{1}^{2}, m_{2}^{2}\right) \sigma(s),
\end{aligned}
$$

and

$$
\sigma(s):=\sqrt{1-\frac{4 m_{\pi}^{2}}{s}} .
$$

We use the log and the square root function both with a cut on the real negative axis.

The triangle function $C$ can be represented by a dispersive integral in the variable $s$ ranging from the two-pion threshold to infinity (unitarity cut):

$$
C(z)=\int_{4 m_{\pi}^{2}}^{\infty} \frac{d s^{\prime}}{\pi} \frac{\operatorname{Im} C\left(s^{\prime}\right)}{s^{\prime}-z}=\int_{4 m_{\pi}^{2}}^{\infty} \frac{d s^{\prime}}{\pi} \frac{\sigma\left(s^{\prime}\right) l\left(s^{\prime}\right)}{s^{\prime}-z}
$$

with

$$
l(s):=-\frac{\pi}{\kappa(s)} \log \frac{Y(s)+\kappa(s)}{Y(s)-\kappa(s)} .
$$

Here $z$ is an arbitrary complex number that does not lie on the unitarity cut, i.e., $z \notin\left[4 m_{\pi}^{2},+\infty[\right.$.

It should be possible to find a dispersive representation of the function $C$ for any values of the masses, but it is necessary to study the cut structure of the logarithm in Eq. (C8). If this cut intersects with the unitarity cut, one needs a proper analytic continuation of the logarithm along the unitarity cut and one picks up an anomalous contribution.

To understand these statements, we consider first the case where (C7) works. In this case, $l(s)$ from (C8) is a smooth function along and in the vicinity of the unitarity cut. Concerning the function $\sigma(s)$, it has a cut for $s \in\left[0,4 m_{\pi}^{2}\right]$. It is convenient to define a function that has a cut along the unitarity cut [72]:

$$
\hat{\sigma}(z):=\sqrt{\frac{4 m_{\pi}^{2}}{z}-1 .}
$$

For $s \in\left[4 m_{\pi}^{2},+\infty[\right.$, it satisfies

$$
\hat{\sigma}(s \pm i \epsilon)=\mp i \sigma(s) .
$$

By construction, the function $C(z)$ is defined via $(\mathrm{C} 7)$ in the whole complex plane except for the unitarity cut. This cut defines a second Riemann sheet. We construct a function $C^{\mathrm{II}}(z)$ that constitutes an analytic continuation of $C$ through the cut. For $s \in\left[4 m_{\pi}^{2},+\infty[\right.$, we demand

$$
\begin{aligned}
C^{\mathrm{II}}(s+i \epsilon) & \stackrel{!}{=} C(s-i \epsilon)=C(s+i \epsilon)-2 i \sigma(s) l(s) \\
& =C(s+i \epsilon)+2 \hat{\sigma}(s+i \epsilon) l(s+i \epsilon) .
\end{aligned}
$$

In the last step, we have used (C10) and the assumption that $l$ is a smooth function around the unitarity cut. This assumption will be critically reviewed below.

For the case at hand, we can use (C11) to define an analytic continuation of $C$ on the second Riemann sheet:

$$
C^{\mathrm{II}}(z):=C(z)+2 \hat{\sigma}(z) l(z) .
$$

The cut structure of $C^{\mathrm{II}}$ originates from the unitarity cut, from the additional cut of $\hat{\sigma}$ along the negative real axis, and from the cut of the logarithm in the expression (C8) for the function $l$. We note that the square root functions that define $\kappa$ in Eq. (C5) and therefore enter (C8) do not cause an additional cut because $l$ is an even function in $\kappa$.

Let us first focus on the unitarity cut. For $s \in\left[4 m_{\pi}^{2},+\infty[\right.$, we find

$$
C^{\mathrm{II}}(s-i \epsilon)=C(s-i \epsilon)+2 \hat{\sigma}(s-i \epsilon) l(s)=C(s+i \epsilon) .
$$

Thus, the unitarity cut connects just the two Riemann sheets.

Next, we focus on the log function. The branch points of the logarithm in Eq. (C8) are given by $Y^{2}(s)=\kappa^{2}(s)$. They 


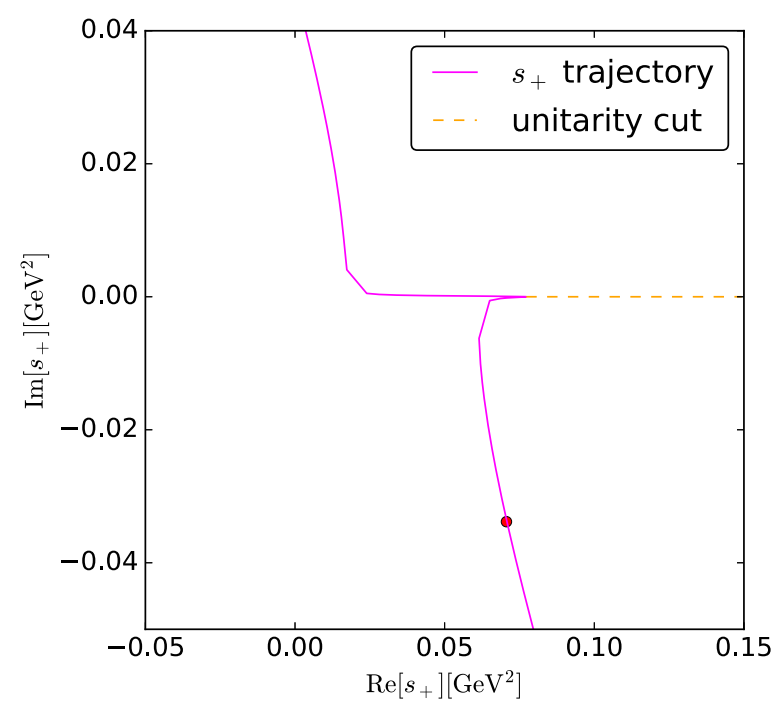

FIG. 10. Real and imaginary part of $s_{+}$obtained by varying $m_{1}^{2}$. The red dot corresponds to $m_{1}^{2}=m_{\Sigma^{*}}^{2}$, which is our case of interest.

are located at

$$
\begin{aligned}
s_{ \pm}= & -\frac{1}{2} m_{\mathrm{exch}}^{2}+\frac{1}{2}\left(m_{1}^{2}+m_{2}^{2}+2 m_{\pi}^{2}\right) \\
& -\frac{m_{1}^{2} m_{2}^{2}-m_{\pi}^{2}\left(m_{1}^{2}+m_{2}^{2}\right)+m_{\pi}^{4}}{2 m_{\mathrm{exch}}^{2}} \\
& \mp \frac{\lambda^{1 / 2}\left(m_{1}^{2}, m_{\mathrm{exch}}^{2}, m_{\pi}^{2}\right) \lambda^{1 / 2}\left(m_{2}^{2}, m_{\mathrm{exch}}^{2}, m_{\pi}^{2}\right)}{2 m_{\mathrm{exch}}^{2}} \\
= & \frac{1}{4 m_{\mathrm{exch}}^{2}}\left\{\left(m_{1}^{2}-m_{2}^{2}\right)^{2}-\left[\lambda^{1 / 2}\left(m_{1}^{2}, m_{\mathrm{exch}}^{2}, m_{\pi}^{2}\right)\right.\right. \\
& \left.\left. \pm \lambda^{1 / 2}\left(m_{2}^{2}, m_{\mathrm{exch}}^{2}, m_{\pi}^{2}\right)\right]^{2}\right\} .
\end{aligned}
$$

The problem is that as a function of the masses, the values of $s_{ \pm}$move through the complex plane that constitutes the second Riemann sheet. If either of the two branch points hits the unitarity cut, then this branch point moves on the physical (=first) Riemann sheet. To be specific, we take $s_{+}$as the solution that has a positive imaginary part for small values of $m_{1}^{2}$. If one replaces $m_{1}^{2}$ by $m_{1}^{2}+i \epsilon$ and follows the motion of $s_{+}$for increasing values of $m_{1}^{2}$, then $s_{+}$moves toward the real axis and could intersect with the unitarity cut. ${ }^{8}$ Figure 10 shows the trajectory of $s_{+}$in the complex plane obtained by varying $m_{1}^{2}$, having fixed $m_{\text {exch }}^{2}=m_{\Sigma}^{2}$ and $m_{2}^{2}=m_{\Lambda}^{2}$. Note the intersection with the unitarity cut, which implies that an additional cut must be located on the first Riemann sheet. The red dot indicates the actual position of $s_{+}$for the physical choice $m_{1}^{2}=m_{\Sigma^{*}}^{2}$.

Indeed, for

$$
m_{1}^{2}+m_{2}^{2}-2 m_{\pi}^{2}-2 m_{\mathrm{exch}}^{2}=0 \quad \text { (cross point) }
$$

\footnotetext{
${ }^{8}$ For completeness, we note that $s_{-}$does not intersect with the unitarity cut and therefore does not enter the first Riemann sheet.
}

the two Källén functions in Eq. (C14) become identical and one finds at this point

$$
\begin{aligned}
\left.s_{+}\right|_{\text {cross point }} & =4 m_{\pi}^{2}, \\
\left.\frac{\partial s_{+}}{\partial m_{1}^{2}}\right|_{\text {cross point }} & =0, \\
\left.\frac{\partial^{2} s_{+}}{\partial\left(m_{1}^{2}\right)^{2}}\right|_{\text {cross point }} & =\frac{2 m_{\pi}^{2}}{\lambda\left(m_{2}^{2}, m_{\text {exch }}^{2}, m_{\pi}^{2}\right)} .
\end{aligned}
$$

Therefore, we obtain

$$
\begin{aligned}
s_{+} & \left.\left(m_{1}^{2}+i \epsilon, \ldots\right)\right|_{\text {cross point }} \\
\approx & {\left[s_{+}\left(m_{1}^{2}, \ldots\right)+i \epsilon \frac{\partial s_{+}\left(m_{1}^{2}, \ldots\right)}{\partial m_{1}^{2}}\right.} \\
& \left.-\frac{1}{2} \epsilon^{2} \frac{\partial^{2} s_{+}\left(m_{1}^{2}, \ldots\right)}{\partial\left(m_{1}^{2}\right)^{2}}\right]_{\text {cross point }} \\
= & 4 m_{\pi}^{2}-\epsilon^{2} \frac{m_{\pi}^{2}}{\lambda\left(m_{2}^{2}, m_{\text {exch }}^{2}, m_{\pi}^{2}\right)} .
\end{aligned}
$$

In other words, the motion of $s_{+}$just turns around (vanishing derivative) at the two-pion threshold. $s_{+}$intersects with the unitarity cut if

$$
\lambda\left(m_{2}^{2}, m_{\mathrm{exch}}^{2}, m_{\pi}^{2}\right)<0 .
$$

One can already see in the original expression (C8) that something goes wrong if $m_{1}^{2}$ becomes so large that (C15) is satisfied. On the real axis, the log in Eq. (C8) is ill defined for $Y(s)=0$. From (C4), we see that this zero of $Y$ is small as long as $m_{1}^{2}$ and $m_{2}^{2}$ are small and $m_{\text {exch }}^{2}$ is large, but the zero of $Y$ reaches the unitarity cut, i.e. the branch point at the twopion threshold for (C15). For even larger values of $m_{1}^{2}$, i.e., for

$$
m_{1}^{2}+m_{2}^{2}-2 m_{\pi}^{2}-2 m_{\mathrm{exch}}^{2}>0,
$$

one needs a smooth analytic continuation of the logarithm along the unitarity cut. Otherwise, the dispersive representation (C7) does not make sense. In addition, (C7) is incomplete, because one has to circumvent also the branch point $s_{+}$, which is now on the physical Riemann sheet. It is convenient to choose the branch that starts at $s_{+}$such that it intersects with the unitarity cut just at its own branch point at the two-pion threshold [69].

The two conditions for $s_{+}$being located on the first Riemann sheet are (C18) and (C19). The dispersive representation of the triangle function $(\mathrm{C} 1)$ is then given by

$$
\begin{aligned}
C(s)= & \frac{1}{2 \pi i} \int d s^{\prime} \frac{\operatorname{disc} C\left(s^{\prime}\right)}{s^{\prime}-s} \\
= & \frac{1}{2 \pi i} \int_{4 m_{\pi}^{2}}^{\infty} d s^{\prime} \frac{\operatorname{disc}_{\text {unit }} C\left(s^{\prime}\right)}{s^{\prime}-s} \\
& +\frac{1}{2 \pi i} \int_{0}^{1} d x \frac{d z(x)}{d x} \frac{\operatorname{disc}_{\text {anom }} C(z(x))}{z(x)-s}
\end{aligned}
$$

with the straight-line path connecting $s_{+}$and the two-pion threshold,

$$
z(x):=(1-x) s_{+}+x 4 m_{\pi}^{2},
$$




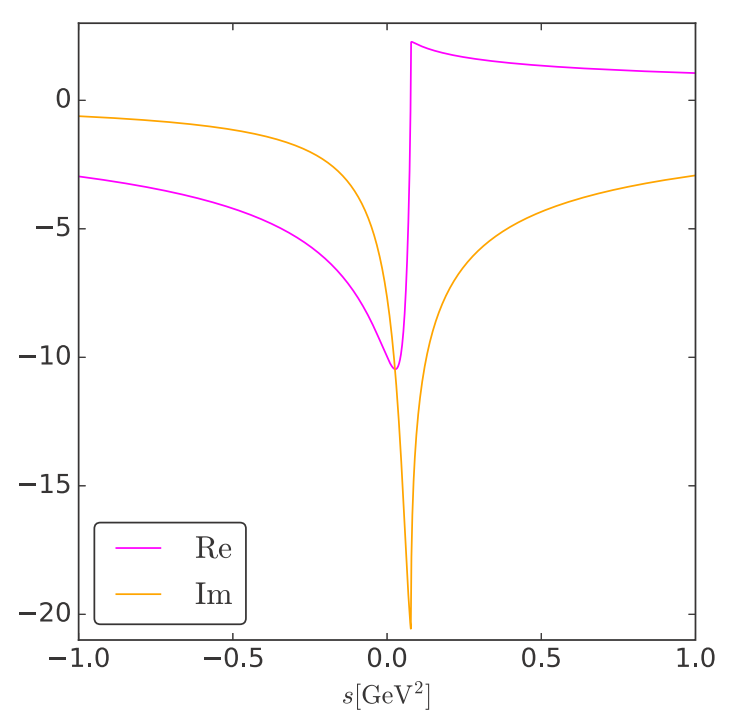

(a)

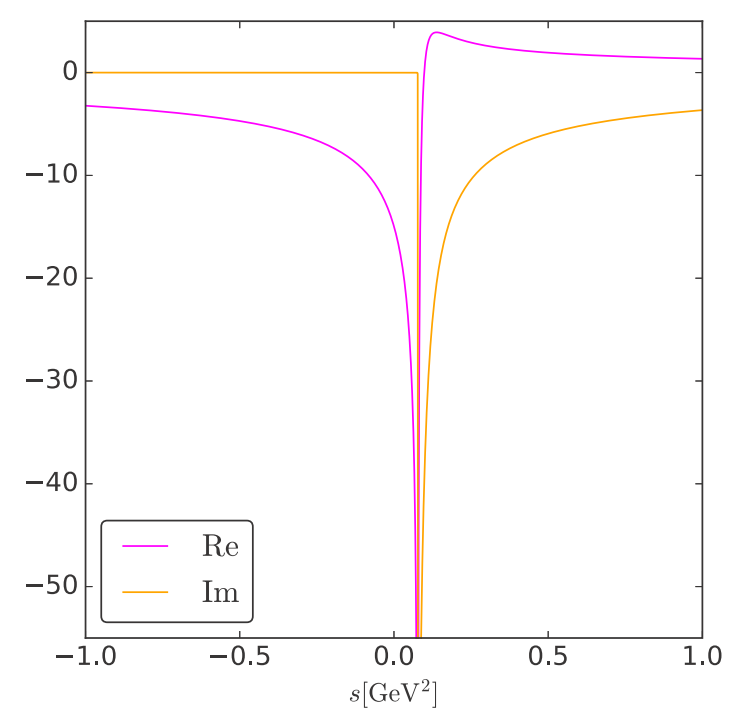

(b)

FIG. 11. Comparison between (a) the triangle function (C1), obtained by either (C2) or (C20), and (b) its incomplete dispersive representation (C7), where only the unitarity cut has been taken into account, neglecting the presence of the anomalous cut. The masses involved here are $m_{1}=m_{\Sigma^{*}}, m_{\mathrm{exch}}=m_{\Sigma}$, and $m_{2}=m_{\Lambda}$.

the function

$$
\operatorname{disc}_{\text {anom }} C(z)=-\frac{4 \pi^{2} i}{\left[-\lambda\left(z, m_{1}^{2}, m_{2}^{2}\right)\right]^{1 / 2}},
$$

and a piecewise defined function given by [cf. (C3)]

$$
\begin{aligned}
\frac{\operatorname{disc}_{\text {unit }} C(s)}{2 i}= & -\pi \frac{\sigma(s)}{\kappa(s)}\left[\log \frac{Y(s)+\kappa(s)}{Y(s)-\kappa(s)}\right. \\
& \left.+2 \pi i \Theta\left(\left(m_{1}-m_{2}\right)^{2}-s\right)\right]
\end{aligned}
$$

for $\lambda\left(s, m_{1}^{2}, m_{2}^{2}\right)>0$ while it is given by

$$
\frac{\operatorname{disc}_{\text {unit }} C(s)}{2 i}=-2 \pi \frac{\sigma(s)}{\tilde{\kappa}(s)}\left[\arctan \frac{\tilde{\kappa}(s)}{Y(s)}+\pi \Theta(-Y(s))\right]
$$

for $\lambda\left(s, m_{1}^{2}, m_{2}^{2}\right)<0$. This function is continuous along the unitarity cut except if $s=\left(m_{1}-m_{2}\right)^{2}$ lies on the cut; there one has an integrable divergence. We have introduced

$$
\tilde{\kappa}(s):=\left[-\lambda\left(s, m_{1}^{2}, m_{2}^{2}\right)\right]^{1 / 2} \sigma(s) .
$$

In Fig. 11(a), the real and imaginary parts of the triangle function (C1) are plotted using $m_{1}=m_{\Sigma^{*}}, m_{\mathrm{exch}}=m_{\Sigma}$, and $m_{2}=m_{\Lambda}$. We have checked that the dispersive representation (C20) for $s+i \epsilon$ with arbitrary real $s$ fully agrees with the direct calculation $(\mathrm{C} 2)$. We want to stress that ignoring the integration along the anomalous cut produces a very incomplete result, shown in Fig. 11(b).

Having established the correct analytic structure, we leave the case of the scalar triangle behind and turn to our TFFs, which have a different partial-wave structure and include the full pion rescattering.

For triangle diagrams with full two-pion rescattering, we extend the usual formulas to allow for the presence of the anomalous cuts. We introduce the values of a function $A$ to the left $\left(A_{+}\right)$and to the right $\left(A_{-}\right)$of a (directed) cut line. The discontinuity of $A$ is then defined by

$$
\operatorname{disc} A:=A_{+}-A_{-} .
$$

For a cut along the real axis, this yields the well-known relations

$$
\begin{aligned}
\operatorname{disc} A(s) & =A(s+i \epsilon)-A(s-i \epsilon) \\
& =A(s+i \epsilon)-A^{*}(s+i \epsilon) \\
& =2 i \operatorname{Im} A(s+i \epsilon) .
\end{aligned}
$$

The optical theorem that leads to (61) and (45) generalizes to

$$
\operatorname{disc} F=2 i \frac{1}{24 \pi} T_{+} \sigma p_{\mathrm{cm}}^{2} F_{\pi-}^{V}
$$

and

$$
\operatorname{disc}(T-K)=2 i T_{+} \sigma t_{-}
$$

with the $p$-wave pion scattering amplitude $t$. Along the unitarity cut, the amplitude $t$ is given by $t=\sin \delta e^{i \delta} / \sigma$.

We recall how (C29) is solved [43]. The Omnès function is introduced as a solution of

$$
\operatorname{disc} \Omega=2 i \Omega_{+} \sigma t_{-} .
$$

This allows us to calculate

$$
\begin{aligned}
\operatorname{disc} \frac{T-K}{\Omega}= & \frac{(T-K)_{+} \Omega_{-}-(T-K)_{-} \Omega_{+}}{\Omega_{+} \Omega_{-}} \\
= & \frac{(T-K)_{+} \Omega_{-}-(T-K)_{+} \Omega_{+}}{\Omega_{+} \Omega_{-}} \\
& +\frac{(T-K)_{+} \Omega_{+}-(T-K)_{-} \Omega_{+}}{\Omega_{+} \Omega_{-}} \\
= & \frac{2 i K_{+} \sigma t_{-}}{\Omega_{-}} .
\end{aligned}
$$


The product $K \sigma$ is essentially proportional to the discontinuity of the triangle function $C$. The proportionality factor $h$ is a rational function of $s$, i.e., has no cuts. With the previous construction of $\operatorname{disc} C$, we have achieved that the two cuts (unitarity cut and anomalous cut) do not intersect. Therefore, we can write for the discontinuity (C31) along the unitarity cut

$$
\operatorname{disc} \frac{T-K}{\Omega}=\frac{2 i K \sigma t_{-}}{\Omega_{-}}=\frac{2 i K \sin \delta}{|\Omega|}
$$

because here $K$ is by construction a continuous function and $\Omega$ has the same phase as $t$. This leads to the standard dispersive part for $T-K$ explicitly given in Eq. (45). Along the anomalous cut, we have

$$
\operatorname{disc} \frac{T-K}{\Omega}=2 i h \operatorname{disc}_{\text {anom }} C \frac{t_{-}}{\Omega_{-}},
$$

which leads to (53).

Finally, we have to solve (C28). For the unitarity cut, we can just integrate the right-hand side of (C28). For the anomalous cut, we use (C29) and find

$$
\operatorname{disc}_{\mathrm{anom}} F=\frac{1}{24 \pi} \operatorname{disc}_{\mathrm{anom}}(T-K) p_{\mathrm{cm}}^{2} \frac{F_{\pi-}^{V}}{t_{-}} .
$$

Since we have a dispersive representation for $T-K$ in Eqs. (45) and (53), we just need to read off the discontinuity along the anomalous cut. This leads to (63).

If one compares the expressions (53) and (63), one notices that (53) looks more complicated with $\Omega$ appearing outside and inside of the integral. Is it not possible to write (53) in a simpler way? After all, $\Omega$ is continuous along the anomalous cut. From (C33), one sees that the discontinuity of $T-K$ along the anomalous cut is indeed just $2 i h \operatorname{disc}_{\text {anom }} C t$. The same can be obtained from (53). But the expression (53) inherits from $\Omega$ also a discontinuity along the unitarity cut. Therefore, a direct dispersive representation of $T-K$ instead of the ratio $(T-K) / \Omega$ leads to an integral where in the integrand the integral of (53) appears. For the form factor, we have this situation of a double integral anyway in Eq. (61) where the integral expressions for $T$ enter in the integrand. But for $T$ itself, one can avoid the double integral representation if one lives with $\Omega$ appearing outside and inside of the integrals.

\section{APPENDIX D: ESTIMATE FOR THE NLO FOUR-POINT PION-BARYON COUPLING CONSTANT}

Ideally, the low-energy constant $c_{F}$ from (83) should be determined from experiment. To have a rough estimate for its size, we apply a vector-meson-dominance (resonancesaturation) assumption [32,61,73,74]. To get a feeling for its accuracy, we will make the same estimate for the octet sector. To this end, we consider the following part of the NLO Lagrangian of [25]:

$$
\begin{aligned}
\mathcal{L}_{V}^{(2)}:= & i c_{M}\left(\mathcal{O}_{\mu \nu}\right)_{d}^{b}\left(f_{+}^{\mu \nu}\right)_{b}^{d}+\text { H.c. } \\
& +\frac{1}{4} c_{F}\left(\mathcal{O}_{\mu \nu}\right)_{d}^{b}\left(\left[u^{\mu}, u^{\nu}\right]\right)_{b}^{d}+\text { H.c. } \\
& +b_{M, D} \operatorname{tr}\left(\bar{B}\left\{f_{+}^{\mu \nu}, \sigma_{\mu \nu} B\right\}\right) \\
& +\frac{i}{2} b_{3,2} \operatorname{tr}\left(\bar{B}\left\{\left[u^{\mu}, u^{\nu}\right], \sigma_{\mu \nu} B\right\}\right)
\end{aligned}
$$

with

$$
\left(\mathcal{O}_{\mu \nu}\right)_{d}^{b}:=\epsilon_{a d e} \bar{B}_{c}^{e} \gamma_{\mu} \gamma_{5} T_{v}^{a b c} .
$$

Estimates for $c_{M}$ and $b_{M, D}$ have been provided in Ref. [25], based on fits to experimental data on radiative decays and magnetic moments, respectively: $\left|c_{M}\right| \approx 1.9 \mathrm{GeV}^{-1}$ and $b_{M, D} \approx 0.32 \mathrm{GeV}^{-1}$.

Vector-meson dominance [32] implies that the coupling strengths of hadrons to two pions (in a $p$ wave) and to photons are correlated. In the $\chi \mathrm{PT}$ framework, this might be rephrased as the statement that the two building blocks $\left[u^{\mu}, u^{\nu}\right]$ and $f_{+}^{\mu \nu}$ appear in a fixed combination, i.e., as the chiral field strength $[61,73]$

$$
\Gamma^{\mu \nu}:=\frac{1}{4}\left[u^{\mu}, u^{\nu}\right]-\frac{i}{2} f_{+}^{\mu \nu} .
$$

Under this assumption, we obtain the following estimates: $c_{F} \approx-2 c_{M}$ and $b_{3,2} \approx b_{M, D}$. In Ref. [74], based on a resonance-saturation approach, the vector-meson contribution to the parameter $b_{3,2}$ (denoted by $b_{10}$ therein) has been estimated to $\approx 0.5 \mathrm{GeV}^{-1}$, i.e., about $50 \%$ larger than our value for $b_{M, D}$. Therefore, we use as an estimate

$$
\left|c_{F}\right|=(4.8 \pm 1.2) \mathrm{GeV}^{-1} \quad \text { and } \quad \frac{c_{F}}{c_{M}}<0
$$

[1] L. G. Landsberg, Phys. Rept. 128, 301 (1985).

[2] E. Czerwiński, S. Eidelman, C. Hanhart, B. Kubis, A. Kupść, S. Leupold, P. Moskal, and S. Schadmand, editors, arXiv:1207.6556 [hep-ph].

[3] G. A. Miller, Phys. Rev. Lett. 99, 112001 (2007).

[4] V. Punjabi, C. F. Perdrisat, M. K. Jones, E. J. Brash, and C. E. Carlson, Eur. Phys. J. A 51, 79 (2015).

[5] J. G. Körner and M. Kuroda, Phys. Rev. D 16, 2165 (1977).

[6] C. E. Carlson, Phys. Rev. D 34, 2704 (1986).
[7] V. Pascalutsa, M. Vanderhaeghen, and S. N. Yang, Phys. Rept. 437, 125 (2007).

[8] E. Kaxiras, E. J. Moniz, and M. Soyeur, Phys. Rev. D 32, 695 (1985).

[9] B. Kubis and U.-G. Meißner, Eur. Phys. J. C 18, 747 (2001).

[10] M. Ablikim et al. (BESIII Collaboration), Phys. Rev. Lett. 123, 122003 (2019).

[11] C. Granados, S. Leupold, and E. Perotti, Eur. Phys. J. A 53, 117 (2017). 
[12] J. M. Alarcón, A. N. Hiller Blin, M. J. Vicente Vacas, and C. Weiss, Nucl. Phys. A 964, 18 (2017).

[13] S. J. Brodsky and G. R. Farrar, Phys. Rev. D 11, 1309 (1975).

[14] G. P. Lepage and S. J. Brodsky, Phys. Lett. B 87, 359 (1979).

[15] S. Scherer and M. R. Schindler, Lect. Notes Phys. 830 (2012).

[16] S. Leupold, Eur. Phys. J. A 54, 1 (2018).

[17] J. M. Alarcón and C. Weiss, Phys. Rev. C 96, 055206 (2017).

[18] J. M. Alarcón and C. Weiss, Phys. Rev. C 97, 055203 (2018).

[19] J. M. Alarcón and C. Weiss, Phys. Lett. B 784, 373 (2018).

[20] M. Hoferichter, J. Ruiz de Elvira, B. Kubis, and U.-G. Meißner, Phys. Rept. 625, 1 (2016).

[21] M. Hoferichter, B. Kubis, J. Ruiz de Elvira, H. W. Hammer, and U.-G. Meißner, Eur. Phys. J. A 52, 331 (2016).

[22] E. E. Jenkins and A. V. Manohar, Phys. Lett. B 259, 353 (1991).

[23] R. F. Dashen and A. V. Manohar, Phys. Lett. B 315, 425 (1993).

[24] T. Ledwig, J. M. Camalich, L. S. Geng, and M. J. Vicente Vacas, Phys. Rev. D 90, 054502 (2014).

[25] M. Holmberg and S. Leupold, Eur. Phys. J. A 54, 103 (2018).

[26] J. F. Donoghue, J. Gasser, and H. Leutwyler, Nucl. Phys. B 343, 341 (1990).

[27] J. F. Donoghue, arXiv:hep-ph/9607351 [hep-ph].

[28] B. Ramstein et al. (HADES Collaboration), EPJ Web Conf. 199, 01008 (2019).

[29] M. F. M. Lutz et al. (PANDA Collaboration), Physics performance report for PANDA: Strong interaction studies with antiprotons, arXiv:0903.3905 [hep-ex].

[30] G. Colangelo, J. Gasser, and H. Leutwyler, Nucl. Phys. B 603, 125 (2001).

[31] R. Garcia-Martin, R. Kaminski, J. R. Pelaez, J. Ruiz de Elvira, and F. J. Yndurain, Phys. Rev. D 83, 074004 (2011).

[32] J. J. Sakurai, Currents and Mesons (University of Chicago Press, Chicago, 1969).

[33] G. Fäldt, Eur. Phys. J. A 52, 141 (2016).

[34] E. Perotti, G. Fäldt, A. Kupść, S. Leupold, and J. J. Song, Phys. Rev. D 99, 056008 (2019).

[35] M. Tanabashi et al. (Particle Data Group), Phys. Rev. D 98 , 030001 (2018).

[36] W. A. Bardeen and W. K. Tung, Phys. Rev. 173, 1423 (1968); Phys. Rev. D 4, 3229(E) (1971).

[37] R. Tarrach, Nuovo Cim. A 28, 409 (1975).

[38] M. E. Peskin and D. V. Schroeder, An Introduction to Quantum Field Theory (Westview Press, Boulder, CO, 1995).

[39] C. Hanhart, Phys. Lett. B 715, 170 (2012).

[40] C. Hanhart, A. Kupść, U.-G. Meißner, F. Stollenwerk, and A. Wirzba, Eur. Phys. J. C 73, 2668 (2013).

[41] M. Fujikawa et al. (Belle Collaboration), Phys. Rev. D 78, 072006 (2008).

[42] N. Muskhelishvili, Singular Integral Equations: Boundary Problems of Functions Theory and Their Application to Mathematical Physics. (Noordhoff, Groningen, Netherlands, 1953).

[43] R. Omnes, Nuovo Cim. 8, 316 (1958).
[44] R. Karplus, C. M. Sommerfield, and E. H. Wichmann, Phys. Rev. 111, 1187 (1958).

[45] M. Dax, T. Isken, and B. Kubis, Eur. Phys. J. C 78, 859 (2018).

[46] M. Hoferichter, B.-L. Hoid, B. Kubis, S. Leupold, and S. P. Schneider, Phys. Rev. Lett. 121, 112002 (2018).

[47] M. Hoferichter, B.-L. Hoid, B. Kubis, S. Leupold, and S. P. Schneider, J. High Energy Phys. 10 (2018) 141.

[48] T. Ledwig, J. Martin-Camalich, V. Pascalutsa, and M. Vanderhaeghen, Phys. Rev. D 85, 034013 (2012).

[49] V. Pascalutsa and M. Vanderhaeghen, Phys. Lett. B 636, 31 (2006).

[50] A. Semke and M. F. M. Lutz, Nucl. Phys. A 778, 153 (2006).

[51] A. J. Buchmann and E. M. Henley, Phys. Lett. B 484, 255 (2000).

[52] A. J. Buchmann and S. A. Moszkowski, Phys. Rev. C 87, 028203 (2013).

[53] V. Pascalutsa and R. Timmermans, Phys. Rev. C 60, 042201(R) (1999)

[54] J. A. Oller, M. Verbeni, and J. Prades, J. High Energy Phys. 09 (2006) 079.

[55] M. Frink and U.-G. Meißner, Eur. Phys. J. A 29, 255 (2006).

[56] J. Gasser and H. Leutwyler, Ann. Phys. 158, 142 (1984).

[57] J. Gasser and H. Leutwyler, Nucl. Phys. B 250, 465 (1985).

[58] S. Scherer, Adv. Nucl. Phys. 27, 277 (2003).

[59] Wolfram Research, Inc., Mathematica, ver. 12.0, Champaign, IL, 2019.

[60] R. Mertig, M. Böhm, and A. Denner, Comput. Phys. Commun. 64, 345 (1991).

[61] G. Ecker, J. Gasser, A. Pich, and E. de Rafael, Nucl. Phys. B 321, 311 (1989).

[62] W. Rarita and J. Schwinger, Phys. Rev. 60, 61 (1941).

[63] G. Penner, Ph.D. thesis, Giessen University, Giessen, Germany, 1999.

[64] S. Stoica, M. F. M. Lutz, and O. Scholten, Phys. Rev. D 84, 125001 (2011).

[65] F. de Jong and R. Malfliet, Phys. Rev. C 46, 2567 (1992).

[66] C. Hacker, N. Wies, J. Gegelia, and S. Scherer, Phys. Rev. C 72, 055203 (2005).

[67] G. 't Hooft and M. J. G. Veltman, Nucl. Phys. B 153, 365 (1979).

[68] W. Lucha, D. Melikhov, and S. Simula, Phys. Rev. D 75, 016001 (2007); 92, 019901(E) (2015).

[69] M. Hoferichter, G. Colangelo, M. Procura, and P. Stoffer, Int. J. Mod. Phys. Conf. Ser. 35, 1460400 (2014).

[70] G. Colangelo, M. Hoferichter, M. Procura, and P. Stoffer, J. High Energy Phys. 09 (2015) 074.

[71] R. E. Cutkosky, J. Math. Phys. 1, 429 (1960).

[72] B. Moussallam, Eur. Phys. J. C 71, 1814 (2011).

[73] G. Ecker, J. Gasser, H. Leutwyler, A. Pich, and E. de Rafael, Phys. Lett. B 223, 425 (1989).

[74] U.-G. Meißner and S. Steininger, Nucl. Phys. B 499, 349 (1997). 